\title{
Poincaré and the Three-Body Problem
}

\author{
Alain Chenciner \\ Observatoire de Paris, IMCCE (UMR 8028), ASD \\ 77, avenue Denfert-Rochereau, 75014 Paris, France \\ chenciner@imcce.fr \\ \& Département de mathématique, Université Paris VII
}

\begin{abstract}
The Three-Body Problem has been a recurrent theme of Poincaré's thought. Having understood very early the need for a qualitative study of "nonintegrable" differential equations, he developed the necessary fundamental tools: analysis, of course, but also topology, geometry, probability. One century later, mathematicians working on the Three-Body Problem still draw inspiration from his works, in particular in the three volumes of Les méthodes nouvelles de la mécanique céleste published respectively in 1892, 1893, 1899.
\end{abstract}

\section{Contents}

\begin{tabular}{lll}
\hline & Introduction & 45
\end{tabular}

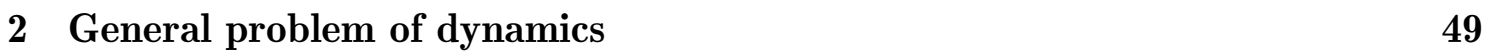

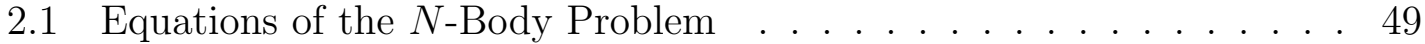

2.2 Kepler Problem: Delaunay and Poincaré coordinates . . . . . . . . . . 50

2.3 Planetary problem: heliocentric coordinates . . . . . . . . . . . . . 51

2.4 Reduced problem . . . . . . . . . . . . . . . . . . . . 53

2.5 General problem of dynamics . . . . . . . . . . . . . . . . . . . . . 54

2.6 The basic intuition . . . . . . . . . . . . . . . . . . . 55

3 Next approximation: Lagrange's and Laplace's secular system 56

3.1 Kepler degeneracy: fast and slow variables . . . . . . . . . . . . . 56

3.2 Averaged system . . . . . . . . . . . . . . . . . . 57

3.3 Quadratic part and singularities . . . . . . . . . . . . . . . 58

\begin{tabular}{|lll}
4 & Periodic solutions 1) Local existence by continuation & 60
\end{tabular}

4.1 Pénétrer dans une place jusqu'ici réputée inabordable . . . . . . . . . 60

4.2 The three "sorts" . . . . . . . . . . . . . . . . . . . . . 62

4.3 and some others . . . . . . . . . . . . . . . . . 65

5 Quasi-periodic solutions 1) Formal aspects: Lindstedt series $\quad 65$

5.1 What is a Lindstedt series? . . . . . . . . . . . . . . . . . . 67

5.2 Different types of Lindstedt series . . . . . . . . . . . . . . . . . . . . 68

5.3 From the old methods to the new ones and back . . . . . . . . . . . . 69

5.4 One torus or a foliation by tori? (Poincaré's ambiguities) . . . . . . . 70

5.5 Existence of the Lindstedt series in the non-degenerate case . . . . . 72

5.6 Coping with the degeneracies ... . . . . . . . . . . . . . . . 75 
$5.7 \quad \ldots$ but forgetting a resonance! $\ldots \ldots$. . . . . . . . . . . . . . . . . . . 77

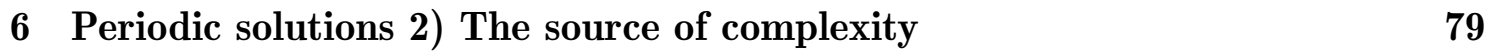

6.1 Variational equations: exponents, stability, asymptotic solutions . . . 79

6.2 Non-existence of uniform first integrals . . . . . . . . . . . . . . . . . 82

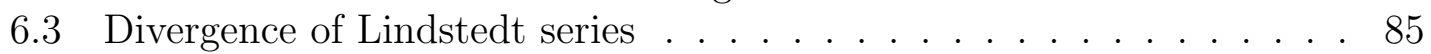

6.4 Back to the old methods: the "Lessons of Celestial Mechanics" . . . . 85

6.5 Complexifying Poincaré's method . . . . . . . . . . . . . . . . 86

\begin{tabular}{llr}
\hline & Resonances 1) Bohlin series & $\mathbf{8 7}$
\end{tabular}

$7.1 \quad$ Circulation, libration, separatrix: what is a Bohlin series? . . . . . . . 87

7.2 "Bohlin method" in the non-degenerate case . . . . . . . . . . . . 88

$\begin{array}{lll}8 & \text { Integral invariants and Poisson stability } & 91\end{array}$

$8.1 \quad$ Integral invariants as integrals of the variational equations . . . . . . 91

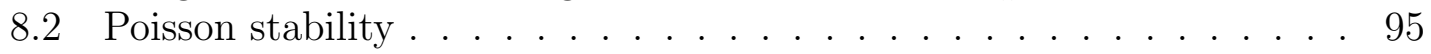

8.3 From the recurrence theorem to ergodic theory . . . . . . . . . . . . 96

$9 \quad$ Stroboscopy 1) Planar Circular Restricted Three-Body Problem 98

9.1 The simplest Three-Body Problem . . . . . . . . . . . . . . . . . . . 98

9.2 Hill stability . . . . . . . . . . . . . . . . . . . . . . . . . . . . . . . . . 99

9.3 Return map . . . . . . . . . . . . . . . . . . . 100

10 Resonances 2) Homoclinic and heteroclinic tangles 102

10.1 Divergence of the Bohlin series and exponentially small splitting . . . 102

10.2 "... cette figure, que je ne cherche même pas à tracer" . . . . . . . . 104

11 Quasi-periodic solutions 2) Analytic aspects: K.A.M. stability 108

11.1 New methods, chapter XIII, section 149 . . . . . . . . . . . 108

11.2 Arnold's theorem for the planar problem . . . . . . . . . . . . . . . . 109

11.3 Dealing with the spatial problem . . . . . . . . . . 110

12 Stroboscopy 2) What we understand of the dynamics of the return map 111

12.1 Bifurcations: subharmonics . . . . . . . . . . . . . . 111

12.2 The last geometric theorem and twist diffeomorphisms of the annulus 114

12.3 Computer experiments and the geometrization of phase space . . . . 116

\begin{tabular}{ll|}
13 A great principle of physics and some collisions & 117
\end{tabular}

13.1 Least action and instability . . . . . . . . . . . . . . . . . . . . 117

13.2 Minimizing the action: the note of 1896 . . . . . . . . . . . . . . . . 118

13.3 Solutions of the second species . . . . . . . . . . . . . . . . 120

$\begin{array}{llr}14 & \text { Resonances 3) diffusion } & 121\end{array}$

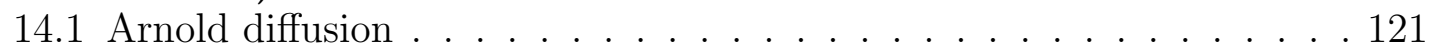

14.2 The oldest open question in dynamical systems . . . . . . . . . . . 121

$\begin{array}{ll}15 \text { Surprises of a eulogy } & 123\end{array}$

\begin{tabular}{ll}
\hline 16 A seminar & 123
\end{tabular} 


\section{Introduction}

Since the time of Newton himself, the Three-Body Problem was a major source of development of analysis: it is enough to mention the names of Euler, Clairaut, d'Alembert, Laplace, Lagrange, Jacobi, Cauchy, ... At the end of the nineteenth century, Poincaré opened a new era, introducing geometric, topological and probabilistic methods in order to understand qualitatively the incredibly complicated behavior of most of the solutions of this problem. At the same time, he analysed the methods used by the astronomers in order to understand the short-term motions using divergent series and, as emphasized in a recent paper by J.P. Ramis [Ra1], he foresaw many aspects for the present development of this theme. From 1883, that is only 4 years after his thesis defense, and until his death in 1912, Poincaré published major papers on (or motivated by) the Three-Body Problem. Already in 1881, in the introduction to the first part of his Mémoire sur les courbes définies par une équation différentielle [P3], he took it as motivation for a qualitative global study:

Prenons, par exemple, le problème des trois corps : ne peut-on pas se demander si l'un des corps restera toujours dans une certaine région du ciel ou bien s'il pourra s'éloigner indéfiniment; si la distance de deux corps augmentera, ou diminuera à l'infini, ou bien si elle restera comprise entre certaines limites ? Ne peut-on pas se poser mille questions de ce genre, qui seront toutes résolues quand on saura construire qualitativement les trajectoires des trois corps ? Et, si l'on considère un nombre plus grand de corps, qu'est-ce que la question de l'invariabilité des éléments des planètes, sinon une véritable question de géométrie qualitative, puisque, faire voir que le grand axe n'a pas de variations séculaires, c'est montrer qu'il oscille constamment entre certaines limites. Tel est le vaste champ de découvertes qui s'ouvre devant les géomètres ${ }^{1}$

In 1885, introducing the third part of the same Mémoire, he was more precise and addressed the reader in his characteristic style:

On n'a pu lire les deux premières parties de ce Mémoire sans être frappé de la ressemblance que présentent les diverses questions qui y sont traitées avec le grand problème astronomique de la stabilité du système solaire. Ce dernier problème est, bien entendu, beaucoup plus compliqué, puisque les équations différentielles du mouvement des corps célestes sont d'ordre très

\footnotetext{
${ }^{1}$ Let us take, for example, the Three-Body Problem: is it not possible to ask whether one of the bodies will remain forever in some region of the sky or whether it will possibly get away indefinitely; whether the distance beween two bodies will increase, or decrease indefinitely, or whether it will stay bounded between some limits? Is it not possible to ask a thousand similar questions, which will all be solved as soon as one is able to construct qualitatively the trajectories of the three bodies? And if one considers more bodies, what is the question of the invariability of the elements of the planets but a true question of qualitative geometry, as showing that the great axis has no secular variations amounts to showing that it oscillates between some limits. Such is the vast field of discoveries which opens up to geometers.
} 
élevé. Il y a même plus, on rencontrera, dans ce problème, une difficulté nouvelle, essentiellement différente de celles que nous avons eu à surmonter dans l'étude du premier ordre, et j'ai l'intention de la faire ressortir, sinon dans cette troisième Partie, du moins dans la suite de ce travail. ${ }^{2}$

The difficulty alluded to by Poincaré is caused by the so-called small denominators which appear in the perturbation series of the astronomers; and indeed, having devoted the end of the Mémoire to the study of the Lindstedt series which govern the problematic existence of families of quasi-periodic solutions "surrounding" a periodic one in $\mathbb{R}^{3}$, he concluded as follows:

D'après ce qui précède, on comprendra sans peine à quel point les difficultés que l'on rencontre en Mécanique céleste, par suite des petits diviseurs et de la quasi-commensurabilité des moyens mouvements, tiennent à la nature même des choses et ne peuvent être tournées. Il est extrêmement probable qu'on les retrouvera, quelle que soit la méthode que l'on emploie 4

A landmark in Poincaré's works on the Three-Body Problem is the famous Memoir Sur le problème des trois corps et les équations de la dynamique [P1], winner in 1889 of the prize given on the occasion of the 60th birthday of the King of Sweden, and even more Les méthodes nouvelles de la mécanique céleste [P2] whose three volumes, totaling almost 1300 pages, appear respectively in 1892, 1893 and 1899. Vastly enlarging the scope of the Memoir, this extraordinary work, which encompasses and develops most of Poincaré's previous researches on the Three-Body Problem, is the source of a major part of the modern theory of Dynamical Systems: normal forms, exponents, invariant manifolds, homoclinic and heteroclinic solutions, analytic nonintegrability, divergence of the perturbation series and exponentially small splitting of separatrices, variational equations and integral invariants, generating functions, recurrence theorem, surfaces of section and return maps, twisting property, all of them are part of the present landscape and they paved the way for bifurcation studies and the theory of singularities, symbolic dynamics, invariant measures and ergodic theory, K.A.M., weak K.A.M. and diffusion, symplectic geometry ... and also a wealth of computer experiments. Indeed, in the realm of Dynamical Systems, "new" ideas which are not in one way or another rooted in Poincaré's works are few and far between ([AKN, HK, C0] $)$. Geometry is a key word but it is amusing to remark how cautiously Poincaré mentions its use in higher dimensions at the beginning of the last part of the Mémoire of 1886 [P3]:

Si l'on veut, dans le cas des équations (2), employer le mode de représentation géométrique dont nous avons fait usage jusqu'ici, il faut regarder $x_{1}, x_{2}, \ldots, x_{n}$ comme les coordonnées d'un point dans l'espace à $n$ dimensions.

\footnotetext{
${ }^{2}$ One cannot have read the first two parts of this Memoir without having been struck by the similarities of the various questions studied there with the great astronomical problem of the stability of the solar system. This last problem is, of course, much more complicated, because the differential equations of motion of the celestial bodies are of a very high order. What is more, one shall encounter in this problem a new difficulty, which differs essentially from the ones we had to overcome in the first-order study, and I intend to bring it out, if not in this third Part, at least in the remainder of this work.

${ }^{3}$ More precisely, families of invariant tori defined by a conserved quantity.

${ }^{4}$ According to what was said above, one will readily appreciate to what extent the difficulties encountered in Celestial Mechanics because of the small denominators and the quasi-commensurability of the mean motions, are attached to the very nature of things and cannot be avoided. It is highly probable that they will come up whatever method we use.
} 
La Géométrie n'est plus alors qu'un langage qui peut être plus ou moins avantageux, ce n'est plus une représentation parlant aux sens. Nous pourrons néanmoins être conduits à employer quelquefois ce langage $!^{5}$

Most of what follows will be dedicated to Les méthodes nouvelles de la mécanique céleste ${ }^{6}$ that is to the perturbative case where a small parameter (of the nature of a mass in the planetary case, the inverse of a distance in the lunar case) controls the proximity to the integrable situation of two uncoupled Kepler Problems.

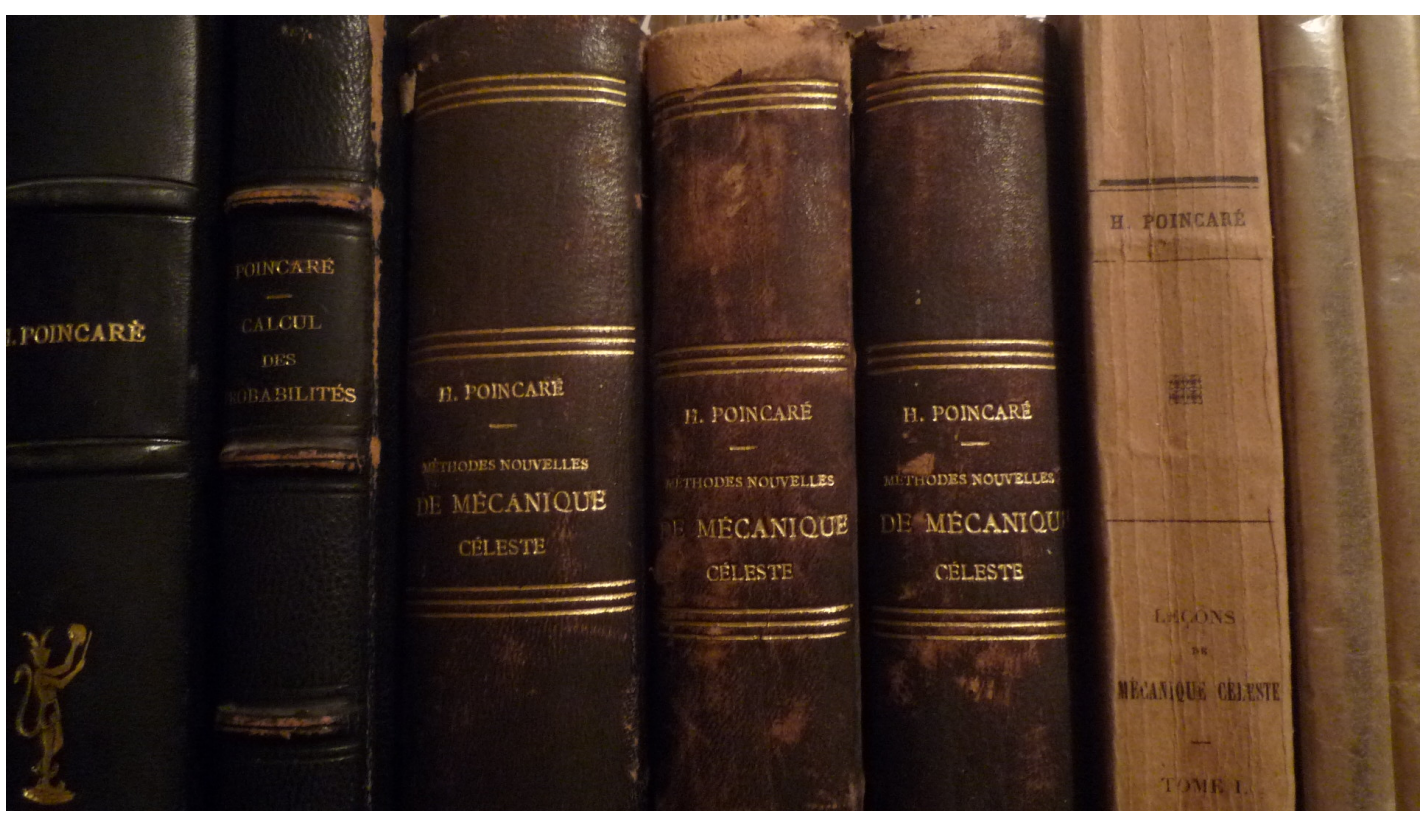

Today, the discovery of extra-solar planetary systems has raised a new interest towards more "exotic", non perturbative situations. I shall in particular mention a short note of 1896 where Poincaré proposed using the variational principle in a global way ${ }^{7}$ in order to find new (relative) periodic solutions of the planar ThreeBody Problem, a method which, as predicted 8 by Poincaré, has turned out to be fruitful [C2].

Warning! The descendence of Poincaré's works around the Three-Body Problem, and in particular The New Methods, is so vast, essentially the whole theory of dynamical systems, that drastic choices had to be made which, necessarily, reflect my own taste. Another solution would have been to imitate "Pierre Menard, autor del Quijote" [Bo]. A third one was chosen by P.J. Melchior who, announcing in the journal "Ciel et Terre" (Vol. 74, p. 290, 1958) the reprinting by Dover (in French, times were different then!) of The New Methods, concludes after some short lines of presentation which essentially amount to giving the subtitles of each volume:

L'ouvrage est en fait trop célèbre et trop bien connu des spécialistes pour que soit nécessaire ici une analyse qui ne pourrait être que fort longue $]^{9}$

\footnotetext{
${ }^{5}$ If one wants to use for equations (2) the geometric way of representation which we have used up to now, one must consider $x_{1}, x_{2}, \ldots, x_{n}$ as the coordinates of a point in an $n$-dimensional space. In this case, Geometry is no more than a language that may be more or less advantageous; it is no longer a representation addressing the senses. Nonetheless, we will occasionally be led to employ this language.

${ }^{6}$ From now on, quoted as The New Methods.

${ }^{7}$ The so-called "direct method" in the Calculus of Variations.

${ }^{8}$ See the quotation in 13.2

${ }^{9}$ The work is in fact too famous and too well-known by specialists to necessitate here an analysis which could not be but very lengthy.
} 


\section{General problem of dynamics}

\subsection{Equations of the $N$-Body Problem}

Denoting by $\vec{r}_{i}, i=0, \ldots, N-1$, the positions of the $N$ bodies ${ }^{10}$ in the ambient Euclidean space $\left(\mathbb{R}^{2}\right.$ or $\left.\mathbb{R}^{3}\right)$ and by $\|$.$\| the Euclidean norm, and setting for convenience$ the gravitational constant equal to 1 , the equations are ${ }^{11}$

$$
m_{i} \frac{d^{2} \vec{r}_{i}}{d t^{2}}=\sum_{j \neq i} \frac{m_{i} m_{j}\left(\vec{r}_{j}-\vec{r}_{i}\right)}{\left\|\vec{r}_{j}-\vec{r}_{i}\right\|^{3}}, \quad i=0, \ldots, N-1 .
$$

Since one can simplify both sides by $m_{i}$, the equations are still meaningful even if some of the masses vanish (Restricted Problems). These equations define a vector field in the phase space (whose coordinates are positions $\overrightarrow{r_{i}}$ and velocities $\overrightarrow{v_{i}}$ of the bodies):

$$
\frac{d \vec{r}_{i}}{d t}=\vec{v}_{i}, \quad \frac{d \vec{v}_{i}}{d t}=\sum_{j \neq i} \frac{m_{i} m_{j}\left(\vec{r}_{j}-\vec{r}_{i}\right)}{\left\|\vec{r}_{j}-\vec{r}_{i}\right\|^{3}}, \quad i=0, \ldots, N-1
$$

For the Three-Body Problem in $\mathbb{R}^{3}$, the problem in $\mathbb{R}^{2}$, and the Restricted Problem, the dimensions of the phase space are respectively $(18,12$ and 4) before reduction of symmetries, $(7,5,3)$ after reduction and fixing of the total energy (see 2.4):

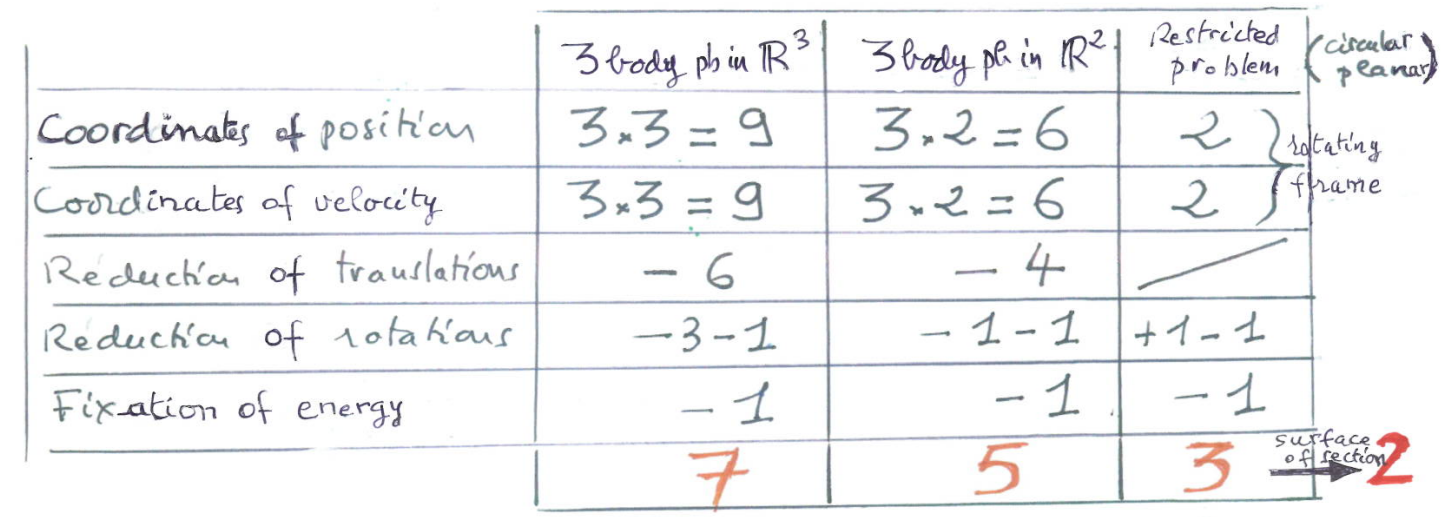

The Hamiltonian (or canonical) form of the equations is obtained by replacing the velocities $\vec{v}_{i}$ by the momenta $\vec{\pi}_{i}=m_{i} \vec{v}_{i}$ and introducing the Hamiltonian (or total energy) $F$. Denoting by $q=\left(\vec{r}_{0}, \ldots, \vec{r}_{N-1}\right)$ the coordinates of position and by $p=\left(\vec{\pi}_{0}, \ldots, \vec{\pi}_{N-1}\right)$ the coordinates of momenta, and looking at the Non-Restricted Problem (for the Restricted Problem, the analogue of $F$ is the Jacobi constant which contains a term coming from the rotation of the frame in which the problem is naturally studied), we get ${ }^{12}$

$$
\frac{d p_{k}}{d t}=-\frac{\partial F}{\partial q_{k}}, \quad \frac{d q_{k}}{d t}=\frac{\partial F}{\partial p_{k}}, \quad \text { where } \quad F=\sum_{i} \frac{1}{2 m_{i}}\left\|\vec{\pi}_{i}\right\|^{2}-\sum_{i<j} \frac{m_{i} m_{j}}{\left\|\vec{r}_{j}-\vec{r}_{i}\right\|} .
$$

\footnotetext{
${ }^{10}$ Being mainly interested in the sequel in the planetary problem, we single out the mass $m_{0}$ which, being that of the Sun, will dominate the other ones.

${ }^{11}$ For a nice, and sometimes surprising, story of these equations, see Alb1].

${ }^{12}$ The sign convention is not Poincaré's own in his statement of the general problem of dynamics (see 2.5 actually, Poincaré is not consistent in his naming of the variables and hence in his choices of signs: denoting by $(x, y)$ the coordinates in phase space, the actions are sometimes represented by $x$ and sometimes by $y$ ).
} 
(Beware that the index $k$ takes values from 1 to 9 if we are looking at the Three-Body Problem in $\mathbb{R}^{3}$.)

The simplest case of interest in classical astronomy is the planetary problem ${ }^{13}$, or $1+2$ body problem, where two planets revolve around a much more massive Sun (a mass ratio $m_{i} / m_{0}=1 / 1000$ in the case of Jupiter). If moreover, as we shall suppose, the two orbits of the planets stay far apart ${ }^{14}$, the equations may be seen as a small perturbation of the limit equations where each planet revolves around the Sun along a Keplerian ellipse unperturbed by the other one. Thus, the first volume of The New Methods starts by recalling the solution of the Two-Body Problem or, what amounts to the same, the Kepler Problem or attraction by a fixed center.

\subsection{Kepler Problem: Delaunay and Poincaré coordinates}

When there are only two bodies, the relative position vector $\vec{r}=\vec{r}_{1}-\vec{r}_{0}$ satisfies the so-called Kepler equation 15

$$
\frac{d^{2} \vec{r}}{d t^{2}}=-\frac{\left(m_{0}+m_{1}\right) \vec{r}}{\|\vec{r}\|^{3}}, \quad \text { with Hamiltonian } \quad h=\frac{1}{2 m}\|\vec{\pi}\|^{2}-\frac{m M}{\|\vec{r}\|},
$$

where we have denoted $M=m_{0}+m_{1}$ and the choice of the reduced mass $m=\frac{m_{0} m_{1}}{m_{0}+m_{1}}$ leaves the potential unchanged. We are only interested here in elliptic solutions, that is motions with negative energy $h$. Introducing Delaunay coordinates

$$
x=(L, G, \Theta), y=(l, g, \theta)
$$

(in the case of the problem in $\mathbb{R}^{3}$, see Figure 1 and [S1] for details ${ }^{16}$ the (still canonical) equations become simply

$$
\frac{d x_{k}}{d t}=-\frac{\partial F}{\partial y_{k}}, \quad \frac{d y_{k}}{d t}=\frac{\partial F}{\partial x_{k}}, \quad \text { where } \quad F=F(x)=-\frac{m^{3} M^{2}}{2 L^{2}} .
$$

Thus the only non-constant quantity is $l$ which varies proportionally to the time and one recovers the three Kepler laws. That the Hamiltonian does not depend on the angles $y=(l, g, \theta)$ but only on the actions $x=(L, G, \Theta)$ means that the Delaunay variables are action-angle coordinates for the Kepler Problem; that it does not depend on $G$ and $\Theta$ reflects the degeneracy of the Kepler Problem: the angles $g$ and $\theta$ stay constant, which means that the ellipse along which the motion takes place has no precession (according to Bertrand's theorem this characterizes Newton's equations and the harmonic oscillator equations among all laws of attraction which are powers of the distance and even all central force laws).

Delaunay coordinates have a major defect for the planetary problem: they do not extend to Keplerian orbits whose eccentricity and inclination vanish. This led Poincaré to introduce a symplectic system of coordinates $((\Lambda, \lambda),(\xi, \eta),(p, q))$, which is obtained from the Delaunay coordinates by, first, making a linear change of coordinates:

$$
\left\{\begin{array}{c}
\Lambda=L, \quad H=L-G, \quad Z=G-\Theta, \\
p \lambda=l+g+\theta, \quad h=-g-\theta, \quad \zeta=-\theta .
\end{array}\right.
$$

\footnotetext{
${ }^{13}$ There is also the lunar problem, to which I shall allude when discussing the Restricted Problem.

${ }^{14}$ This excludes in particular the Lagrange equilateral solutions for which the limit orbits share the same value of the semi-major axis.

15 which, of course, Kepler never wrote.

${ }^{16}$ Neither the sign conventions nor the constants are the same as those of Poincaré who worked in conformal symplectic coordinates and not in symplectic ones.
} 
and then considering the pairs $(H, h)$ et $(Z, \zeta)$ as planar symplectic polar coordinates:

$$
\begin{cases}\sqrt{2 H} \cos h=\xi, & \sqrt{2 H} \sin h=\eta \\ \sqrt{2 Z} \cos \zeta=p, & \sqrt{2 Z} \sin \zeta=q\end{cases}
$$

Called today Poincaré coordinate $\$ 17$, these coordinates are pertinent in the neighborhood of zero eccentricity $(H=0)$ or zero inclination $(Z=0)^{18}$. The conjugate variables $(\Lambda, \lambda)$ respectively describe the semi-major axis (i.e., the energy) and the mean longitude (i.e., the position of the body on its elliptic trajectory), while the conjugate variables $(\xi, \eta)$ describe the position of the ellipse within its plane and its eccentricity, and the set of conjugate variables $(p, q)$ (not to be confused with the position and momentum coordinates introduced in 2.1) describe the position of the plane containing the ellipse and its inclination with respect to the reference plane.

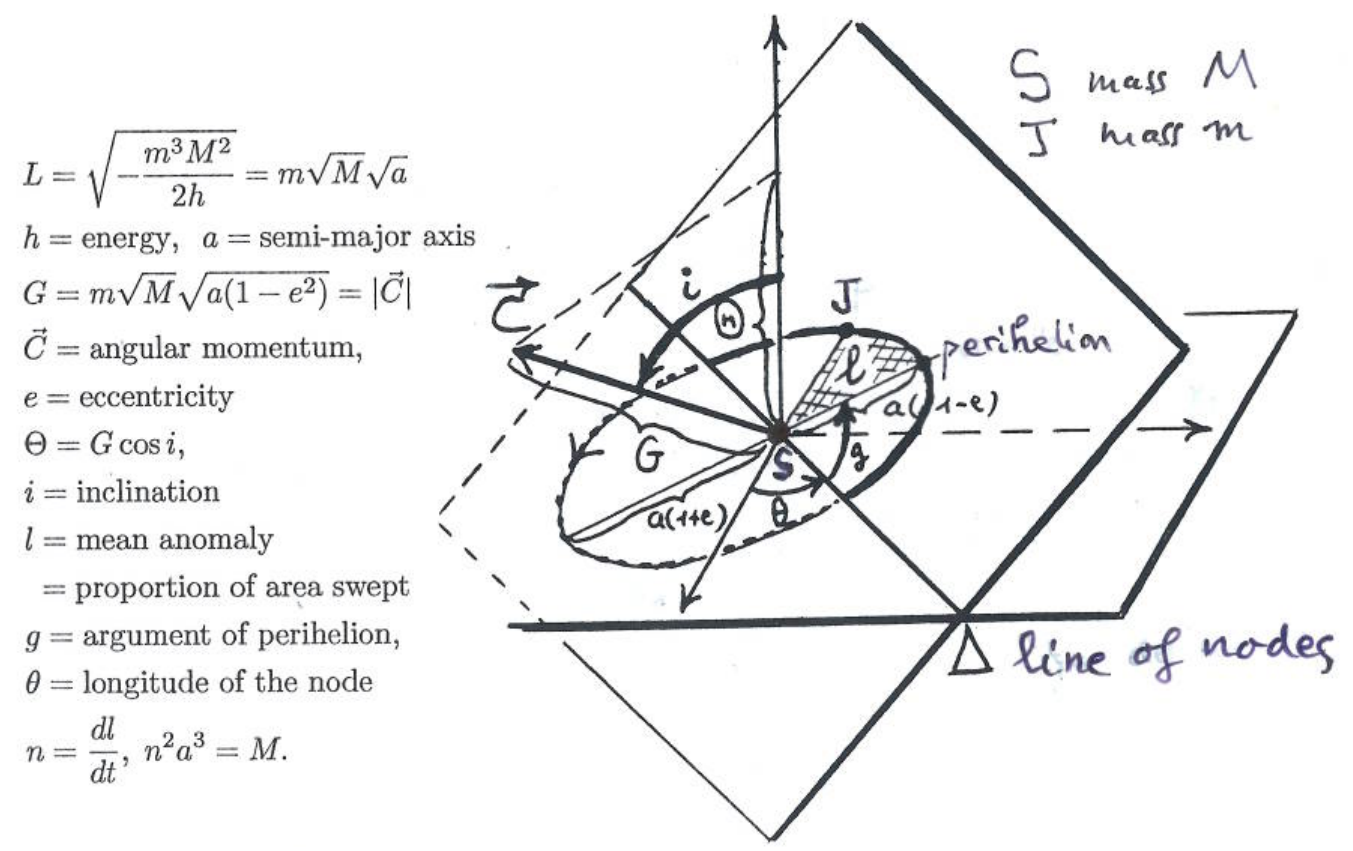

Figure 1: Delaunay coordinates.

\subsection{Planetary problem: heliocentric coordinates}

Reducing the Galilean symmetry by the use of Jacobi coordinates (as Poincaré did in the first chapter of The New Methods) or canonical heliocentric coordinates (that he will introduce only in 1896, see [S1] for a detailed study of both) one writes the planetary problem as a perturbation of two uncoupled fictitious Kepler Problems.

Let us choose for instance the canonical heliocentric coordinates:

$$
\begin{cases}\vec{Q}_{0}=\vec{r}_{0}, & \vec{Q}_{i}=\vec{r}_{i}-\vec{r}_{0}, i=1,2, \\ \vec{P}_{0}=\vec{\pi}_{0}+\vec{\pi}_{1}+\vec{\pi}_{2}, & \vec{P}_{i}=\vec{\pi}_{i}, i=1,2 .\end{cases}
$$

\footnotetext{
${ }^{17}$ The coordinates we have defined are adapted to solutions which turn in the positive, prograde, direction. One can introduce in the same way Poincaré coordinates adapted to retrograde motions.

${ }^{18}$ In the first volume of the Lessons [P12], Poincaré gived a detailed proof of the analyticity of these coordinates.
} 
The Hamiltonian

$$
\sum_{i=0}^{2} \frac{\left|p_{i}\right|^{2}}{2 m_{i}}-\sum_{0 \leq i<j \leq 2} \frac{m_{i} m_{j}}{\left|q_{j}-q_{i}\right|}
$$

of the $1+2$ body problem becomes (after setting $P_{0}=0$, which amounts to fixing the center of mass)

$$
\left\{\begin{array}{l}
\mathcal{H}\left(\vec{P}_{1}, \vec{P}_{2}, \vec{Q}_{1}, \vec{Q}_{2}\right)=\mathcal{H}(\vec{P}, \vec{Q})=\mathcal{H}_{0}(\vec{P}, \vec{Q})+\mathcal{H}_{1}(\vec{P}, \vec{Q}) \\
\mathcal{H}_{0}=\sum_{i=1}^{2}\left|\vec{P}_{i}\right|^{2}\left(\frac{1}{2 m_{0}}+\frac{1}{2 m_{i}}\right)-\sum_{i=1}^{2} \frac{m_{0} m_{i}}{\left|\vec{Q}_{i}\right|} \\
\mathcal{H}_{1}=\mathcal{H}_{1 p}+\mathcal{H}_{1 c}=-\frac{m_{1} m_{2}}{\left|\vec{Q}_{2}-\vec{Q}_{1}\right|}+\frac{\vec{P}_{1} \cdot \vec{P}_{2}}{m_{0}}
\end{array}\right.
$$

The equations (written in a concise way which, as Poincaré would say, "ne pouvait d'ailleurs tromper personne" 19

$$
\frac{d \vec{P}_{i}}{d t}=-\frac{\partial \mathcal{H}}{\partial \vec{Q}_{i}}, \quad \frac{d \vec{Q}_{i}}{d t}=\frac{\partial \mathcal{H}}{\partial \vec{P}_{i}}
$$

may be interpreted as describing the motion of two fictitious planets around a fixed center. The motions corresponding to the unperturbed Hamiltonian $H_{0}$ are those of two uncoupled Kepler Problems for which the mass of the fixed center and the mass of the planet are respectively

$$
\bar{M}_{i}=m_{0}+m_{i} \text { and } \bar{m}_{i}=\frac{m_{0} m_{i}}{m_{0}+m_{i}}, \quad i=1,2 .
$$

The perturbing function is the sum of a principal part $H_{1 p}$ and a complementary part $H_{1 c}$. It remains small as long as $m_{0}$ is much larger than $m_{1}, m_{2}$ and the two unperturbed ellipses are well separated.

Recall the conservation of angular momentum 20 .

$$
\vec{C}=\sum_{i=0}^{2} m_{i} \vec{r}_{i} \wedge \frac{d \vec{r}_{i}}{d t}=\sum_{i=0}^{2} \vec{r}_{i} \wedge \vec{\pi}_{i}=\sum_{i=0}^{2} \vec{Q}_{i} \wedge \vec{P}_{i}=\sum_{i=1}^{2} \vec{Q}_{i} \wedge \vec{P}_{i}
$$

In $\mathbb{R}^{3}$, the (invariant) plane orthogonal to $\vec{C}$ (when $\vec{C} \neq 0$ ) is called the Laplace plane. The expression of the angular momentum as the sum of the angular momenta of the two fictitious bodies with position $\vec{Q}_{i}$ and momentum $\vec{P}_{i}, i=1,2$, shows that the Laplace plane and the two planes spanned respectively by $\left(\vec{Q}_{1}, \vec{P}_{1}\right)$ and $\left(\vec{Q}_{2}, \vec{P}_{2}\right)$ have a common intersection, called the line of nodes as in Figure 2. We may now introduce Delaunay coordinates $\left(L_{1}, G_{1}, \Theta_{1}, l_{1}, g_{1}, \theta_{1}\right),\left(L_{2}, G_{2}, \Theta_{2}, l_{2}, g_{2}, \theta_{2}\right)$, for each one of the Kepler Problems ${ }^{21}$. As Poincaré explains in the first chapter of [P2] $]^{22}$.

\footnotetext{
${ }^{19}$ could not anyway mislead anyone (compare with the beginning of section 341 of The New Methods).

${ }^{20}$ The angular momentum is a bivector but, in $\mathbb{R}^{3}$ (resp. $\mathbb{R}^{2}$ ) endowed with its canonical orientation and canonical Euclidean structure, it may be identified with a vector (resp. a real number).

${ }^{21}$ which give slightly different masses $\tilde{M}_{1}$ and $\tilde{M}_{2}$ to the Sun.

${ }^{22}$ Since Poincaré used Jacobi coordinates and numbers 1,2,3 the bodies, I needed to change the mass of the Sun in the quotation which originally had $m_{1}+m_{2}$. Astronomers used to dislike heliocentric coordinates because the fictitious ellipses are not osculating the real trajectories, but this is not significant.
} 
Si alors, à un instant quelconque, les forces appliquées à la première masse fictive venaient à disparaître, et qu'elles soient remplacées par l'attraction d'une masse $m_{0}+m_{1}$ placée à l'origine, cette masse se mouvrait suivant les lois de Képler et les éléments de ce mouvement képlérien seraient $L, G, \Theta, l, g$ et $\theta .23$

Warning! We shall choose, as is usual (see for instance [Ti, Cha]), to define $\theta_{j}$ as the longitude of the ascending node of the $j$ th planet. This implies $\theta_{2}-\theta_{1}=\pi \bmod 2 \pi$. On the contrary, in section 16 , Poincaré writes that $\theta_{1}=\theta_{2}$ without making explicit the fact that there is a choice.

Setting

$$
x=\left(L_{1}, G_{1}, \Theta_{1}, L_{2}, G_{2}, \Theta_{2}\right), y=\left(l_{1}, g_{1}, \theta_{1}, l_{2}, g_{2}, \theta_{2}\right),
$$

the equations are still canonical with a Hamiltonian of the form

$$
\frac{K_{1}}{L_{1}^{2}}+\frac{K_{2}}{L_{2}^{2}}+R(x, y)
$$

where $K_{1}, K_{2}$ are constants and the perturbing function $R$ depends on all the variables.

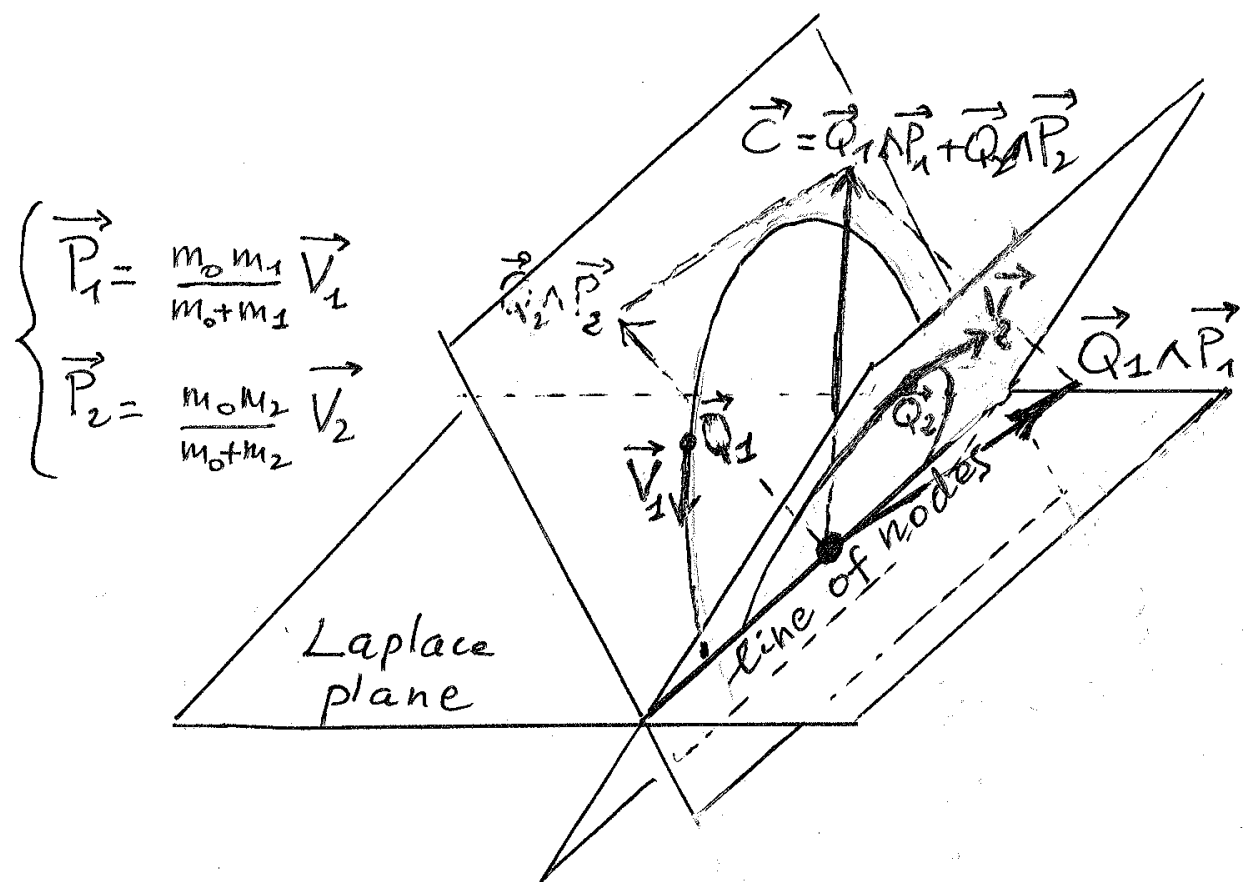

Figure 2: The fictitious Keplerian motions at some instant.

\subsection{Reduced problem}

When fixing the direction of the angular momentum to be vertical in the preceding section, we have already performed a partial reduction ${ }^{24}$ of the rotational symmetry,

\footnotetext{
${ }^{23}$ If, at some instant, the forces which are applied to the first fictitious mass happened to vanish, and if they were replaced by the attraction of a mass $m_{0}+m_{1}$ located at the origin, this mass would move according to Kepler laws and the elements of this Keplerian motion would be $L, G, \Theta, l, g$ and $\theta$.

${ }^{24}$ Partial reduction will play an important role in section 11.3
} 
which amounts to restricting the dynamics to the (symplectic) invariant submanifold of the phase space defined by

$$
\theta_{2}-\theta_{1}=\pi, \quad G_{2}^{2}-\Theta_{2}^{2}=G_{1}^{2}-\Theta_{1}^{2} .
$$

Since fixing the norm of the angular momentum is equivalent to setting $\Theta_{1}+\Theta_{2}=C$, taking the time derivative, we get that $\frac{\partial F}{\partial \theta_{1}}+\frac{\partial F}{\partial \theta_{2}}=0$, from which it follows that $F$ depends only on the difference $\theta_{2}-\theta_{1}$. Since this difference is constant, $F$ does not depend on $\theta_{1}, \theta_{2}$ once the angular momentum is fixed, and we can perform a complete symplectic reduction by keeping only the 8 variables

$$
L_{1}, L_{2}, l_{1}, l_{2}, G_{1}, G_{2}, g_{1}, g_{2}
$$

from which one recovers the remaining variables. This is the Jacobi reduction of the node. In the case of the planar problem, the reduced problem is obtained by taking the 6 variables

$$
L_{1}, L_{2}, l_{1}, l_{2}, H=G_{1}, h=g_{1}-g_{2} .
$$

\subsection{General problem of dynamics}

Introducing a small parameter $\mu$ and supposing that $m_{i}=\mu \bar{m}_{i}, i=1,2$, where $m_{0}, \bar{m}_{1}, \bar{m}_{2}$ are of order 1 , and dividing the Hamiltonian (reduced or not reduced) by $\mu$, one gets a Hamiltonian $F_{0}(x)+\mu F_{1}(x, y)$ of the form which Poincaré, in section 13 of chapter I of [P2], calls the general problem of dynamics:

Nous sommes donc conduit à nous proposer le problème suivant:

Etudier les équations canonique ${ }^{25}$

$$
\frac{d x_{i}}{d t}=\frac{d F}{d y_{i}}, \quad \frac{d y_{i}}{d t}=-\frac{d F}{d x_{i}},
$$

en supposant que la fonction $F(x, y, \mu)$ peut se développer suivant les puissances d'un paramètre très petit $\mu$ de la manière suivante;

$$
F=F_{0}+\mu F_{1}+\mu^{2} F_{2}+\cdots,
$$

en supposant de plus que $F_{0}$ ne dépend que des $x$ et est indépendant des $y$ et que $F_{1}, F_{2}, \cdots$ sont des fonctions périodiques de période $2 \pi$ par rapport aux $y \stackrel{26}{16}$

In other words, the system is a small perturbation of the completey integrable system associated with $F_{0}(x)$, for which $(x, y)$ are action-angle coordinates and whose solutions are:

$$
x_{i}=x_{i}^{0}, \quad y_{i}=n_{i}^{0} t+y_{i}^{0}, \text { where } n_{i}^{0}=-\frac{d F_{0}}{d x_{i}}\left(x^{0}\right) .
$$

${ }^{25}$ Here, as I already said, Poincaré's sign conventions are different from the ones I have chosen.

${ }^{26}$ Hence we are led to propose the following problem: study the canonical equations

$$
\frac{d x_{i}}{d t}=\frac{d F}{d y_{i}}, \quad \frac{d y_{i}}{d t}=-\frac{d F}{d x_{i}}
$$

while supposing that the function $F(x, y, \mu)$ may be expanded in powers of a very small parameter $\mu$ in the following way;

$$
F=F_{0}+\mu F_{1}+\mu^{2} F_{2}+\cdots,
$$

with moreover the hypothesis that $F_{0}$ depends only on the $x$ variables and is independent of the $y$ and that $F_{1}, F_{2}, \cdots$ are $2 \pi$-periodic functions of the $y$ 's. 
For this completely integrable system, the phase space, which is the product $\mathbb{T}^{N} \times \mathbb{R}^{N}$ (coordinates $(y, x))$ of a torus $\mathbb{T}^{N}=\mathbb{R}^{N} /(2 \pi \mathbb{Z})^{N}=(\mathbb{R} / 2 \pi \mathbb{Z})^{N}$ by a vector space ${ }^{27}$ is foliated by $N$-dimensional invariant tori $x=x^{0}=\left(x_{1}^{0}, \cdots, x_{N}^{0}\right)$ on which the integral curves are quasi-periodic with frequencies $n_{i}$ which depend only on $x^{0}$. If this dependance is effective, there is a dense subfamily of these tori bearing dense solutions ${ }^{28}$ and another dense subfamily of these tori which are foliated by periodic solutions. This fact lies at the root of the complexity of the perturbations of such systems; we shall give in the next section an intuitive explanation of why it is so.

\subsection{The basic intuition}

The main idea underlying Poincaré's work in The New Methods is that an invariant torus of an integrable approximation which is the union of periodic solutions (a so-called completely resonant torus) has no dynamical significance and hence has a great probability of being destroyed by a general enough perturbation, giving rise to "generic" (i.e., with non-zero exponents, see section 6.1) periodic solutions which are the main reason for non-integrability (see section 6.2 ) and for the divergence of Lindstedt series with variable frequencies (see section 6.3)

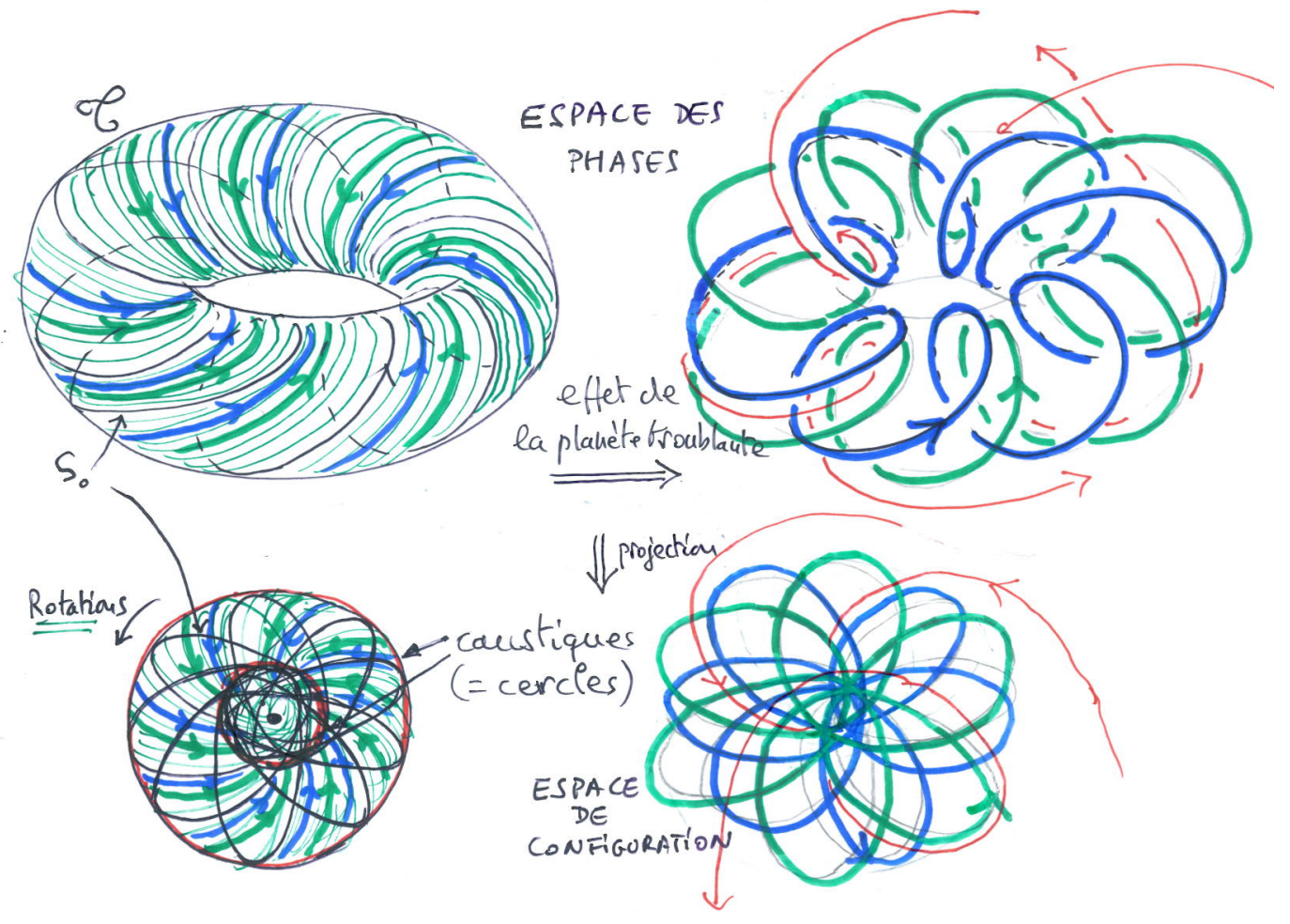

Figure 3: The breaking of a resonant torus.

In contrast, results of the KAM type dwell on the fact that, being the closure of any one of the solutions it bears, a non-resonant quasi-periodic invariant torus has dynamical significance and hence has some chance of resisting a small enough generic perturbation (and indeed it does, provided the solutions are "sufficiently dense", see section 11).

\footnotetext{
${ }^{27}$ or, more conceptually, the cotangent bundle of a torus (the $y_{i}$ are angular configuration coordinates on the torus and the $x_{i}$ are momentum coordinates).

${ }^{28}$ More precisely, each one of these tori is the closure of any one of the solutions it contains.
} 


\section{Next approximation: Lagrange's and Laplace's secular system}

Since Newton's study of the slow motion of the Moon's node and studies by Euler, Clairaut and d'Alembert of the slow motion of its apogee, the long-term evolution of eccentricities and of inclinations, perihelia and nodes is a fundamental object of observation. Because of the degeneracy of the Kepler Problem, the time scale of their evolution is much slower than that of the semi-major axes and mean longitudes ${ }^{29}$ This makes it reasonable, in the first approximation, to force the decoupling of these two kinds of variations by averaging out the fast oscillations. Doing so, one gets a closed system, the averaged system or (first-order) secular system, which describes the long-term evolution of the shape and position of the ellipses osculating to the trajectories of the planets. The linearization at the circular and horizontal motions of this system was studied with great success by Lagrange and Laplace and is at the origin of the first "stability result" of a planetary system. On the other hand, the numerical studies of Jacques Laskar have shown the relevance of the secular system (in fact a higher-order version) for long-term simulations of the evolution of the solar system (see [Las1]). In his studies of periodic and quasi-periodic solutions of the Three-Body Problem, Poincaré had to rely on properties of the averaged system for which he referred to Tisserand [Ti]. In this section, I give the necessary notions on this system.

\subsection{Kepler degeneracy: fast and slow variables}

The very simplicity of the solutions of the Kepler Problem is one main source of complexity of the Three-Body Problem (at least of the $1+2$ body problem): indeed, all Kepler elliptic motions with the same energy are periodic with the same period. This corresponds to the fact that the Hamiltonian depends only on one of the action variables $x$, namely $L$, and implies that, for the Kepler Problem in $\mathbb{R}^{3}$, the invariant tori foliating a negative energy manifold are periodic solutions, i.e., of dimension 1 instead of being of dimension 3 as would be the case "in general": the situation is completely resonant. In fact, using a Poincaré return map (see section 9.3), the dynamical study of the Kepler Problem in a given negative energy may be reduced to the identity map. This degeneracy disappears for the Restricted Problem (see section 9.1) where the unperturbed system is the Kepler Problem in a rotating frame. For the planetary problem, the difficulty is solved by finding better, and in particular non-degenerate, approximations called the secular systems which give a first approximation to the slow variation of the variables $\left(G_{1}, \Theta_{1}, G_{2}, \Theta_{2}, g_{1}, \theta_{1}, g_{2}, \theta_{2}\right)$ over the time scale of centuries, hence their name of slow (or secular) variables while $\left(L_{1}, L_{2}, l_{1}, l_{2}\right)$ are called fast variables.

When eccentricities and inclinations are close to 0 , one replaces Delaunay coordinates by Poincaré coordinates (see 2.2), the fast variables being now $\left(\Lambda_{1}, \Lambda_{2}, \lambda_{1}, \lambda_{2}\right)$ and the slow ones being conveniently written in complex notation

$$
\left\{\begin{array}{l}
u_{j}=\xi_{j}+i \eta_{j}=\sqrt{2 H_{j}} e^{i h_{j}}=\sqrt{\Lambda_{j}} e_{j} e^{-i\left(g_{j}+\theta_{j}\right)}+\cdots \\
z_{j}=p_{j}+i q_{j}=\sqrt{2 Z_{j}} e^{i \zeta_{j}}=\sqrt{\Lambda_{j}} i_{j} e^{-i \theta_{j}}+\cdots
\end{array}\right.
$$

\footnotetext{
${ }^{29}$ In fact, it can happen that "short-term" periods become comparable to "long-term" ones: an example is the great inequality, whose period is approximately 900 years, of Jupiter and Saturn, due to a near resonance in their mean motions (see Las1 for the fascinating story of the explanation of this inequality).
} 


\subsection{Averaged system}

Given the Hamiltonian

$$
F\left(\Lambda_{1}, \Lambda_{2}, \lambda_{1}, \lambda_{2}, u_{1}, u_{2}, z_{1}, z_{2}\right)=F_{0}\left(\Lambda_{1}, \Lambda_{2}\right)+\mu F_{1}\left(\Lambda_{1}, \Lambda_{2}, \lambda_{1}, \lambda_{2}, u_{1}, u_{2}, z_{1}, z_{2}\right)
$$

of the planetary problem, one defines the averaged Hamiltonian (or first-order secular Hamiltonian) by averaging with respect to the fast angles $\lambda_{1}, \lambda_{2}$ :

$\tilde{F}_{\left(\Lambda_{1}, \Lambda_{2}\right)}\left(u_{1}, u_{2}, z_{1}, z_{2}\right)=F_{0}\left(\Lambda_{1}, \Lambda_{2}\right)+\frac{\mu}{(2 \pi)^{2}} \int_{\mathbb{T}^{2}} F_{1}\left(\Lambda_{1}, \Lambda_{2}, \lambda_{1}, \lambda_{2}, u_{1}, u_{2}, z_{1}, z_{2}\right) d \lambda_{1} d \lambda_{2}$.

Once $\Lambda_{1}$ and $\Lambda_{2}$ are fixed, the averaged system is defined on the space of all pairs of ellipses with the same focus and the same value of the semi-major axes. Forgetting the fast variations around the average, the system describes the slow evolution of the eccentricities and the longitudes of the perihelia $\left(u_{1}, u_{2}\right)$ of the two ellipses on the one hand, of the inclinations and the longitudes of their nodes $\left(z_{1}, z_{2}\right)$ on the other hand. The study of this system in the neighborhood of circular coplanar ellipses with the same orientation played a major role in the classical studies of the problem by astronomers and mathematicians, especially at the end of the 18th and beginning of the 19th century with the fundamental works of Lagrange and Laplace. We shall see how it appears naturally as an approximation to the true equations when studying in 5.5 and 5.6 the way Poincaré handles the Lindstedt series.

In terms of the original cartesian coordinates, on checks that

$$
\tilde{F}=F_{0}-\frac{1}{(2 \pi)^{2}} \int_{\mathbb{T}^{2}} \frac{m_{1} m_{2}}{\left|Q_{2}-Q_{1}\right|} d \lambda_{1} d \lambda_{2}
$$

Indeed, the reader will check that the average of the complementary part of the perturbing function vanishes. The constant $F_{0}$ does not affect the dynamics, hence the secular Hamiltonian describes the evolution of the shapes and positions of massive ellipses whose mass would be allocated according to Kepler's second law. By construction, it does not depend on the angles $\lambda_{i}$ and hence its flow fixes the $\Lambda_{i}$, that is the semi-major axes of the ellipses. This is the first part of the Laplace stability theorem. From now on, we shall consider the averaged Hamiltonian as defined on a domain of $\mathbb{C}^{4}=\mathbb{R}^{8}$ (coordinates $\left(u_{1}, u_{2}, z_{1}, z_{2}\right)$ depending on "parameters" $\Lambda_{1}, \Lambda_{2}$ which are fixed in a "sensible" way (that is in such a way that the two ellipses never get too close to each other, which ensures that the perturbing function is indeed a small perturbation).

The three area integrals, that is the three components of the angular momentum, are still first integrals of the secular Hamiltonian. If one fixes the angular momentum in the direction of the vertical axis, they become

$$
\left\{\begin{array}{l}
\sum_{j=1}^{n}\left(\Lambda_{j}-\frac{1}{2}\left(\left|u_{j}\right|^{2}+\left|z_{j}\right|^{2}\right)\right)=\text { a positive real constant } \\
\sum_{j=1}^{n} z_{j} \sqrt{2 \Lambda_{j}-\left|u_{j}\right|^{2}-\frac{1}{2}\left|z_{j}\right|^{2}}=0 \in \mathbb{C}
\end{array}\right.
$$

(the reader too lazy to do the computation may look at the first volume of the Leçons de Mécanique Céleste ([P12] section 144). 
The $\Lambda_{j}$ being constants, the first equation defines a sphere $S^{7}$ in $\mathbb{R}^{8}=\mathbb{C}^{4}$ surrounding the singularity at the origin, corresponding to a pair of horizontal circles; this is the second part of Laplace stability theorem: at the secular level, the eccentricities and inclinations remain small for all time if they are small initially. If the radius $\epsilon$ of this sphere is chosen sufficiently small, the submanifold $J^{5}$ of $\mathbb{R}^{8}$ defined by the three equations above is diffeomorphic to the intersection of $S^{7}$ with the complex hyperplane whose equation is $\sum_{i=1}^{2} z_{i} \sqrt{2 \Lambda_{i}}=0$, that is to $S^{5}$. A quick inspection of Poincaré coordinates shows that the groupe of rotations around $O x_{3}$ acts diagonally on $\mathbb{R}^{8}=\mathbb{C}^{4}$ :

$$
e^{i \alpha} \cdot\left(u_{1}, u_{2}, z_{1},, z_{2}\right)=\left(u_{1} e^{i \alpha}, u_{2} e^{i \alpha}, z_{1} e^{i \alpha}, z_{2} e^{i \alpha}\right) .
$$

Hence the quotient $K^{4}$ of $J^{5}$ by this action (which preserves it) is diffeomorphic to the quotient of the round sphere $S^{5}$, that is to the complex projective space $P^{2}(\mathbb{C})$. Note that $K^{4}$ is naturally a submanifold of the quotient $P^{3}(\mathbb{C})$ of $S^{7}$ on which one can use the homogeneous complex coordinates $u_{1}: u_{2}: z_{1}: z_{2}$, and that the invariant submanifold defined by the motions in the horizontal plane coincides with the subspace $P^{1}(\mathbb{C})$ (= the Riemann sphere) obtained by setting $z_{1}=z_{2}=0$ (and on which $u_{1}: u_{2}$ are homogeneous coordinates). Finally, after reduction of the rotation symmetry, the secular spatial (resp. planar) $1+2$ body problem amounts to the study of a Hamiltonian system on a submanifold $K^{4}$ (resp. $K^{2}$ ) of $P^{3}(\mathbb{C})$ $\left(\right.$ resp. $\left.P^{2}(\mathbb{C})\right)$ diffeomorphic to $P^{2}(\mathbb{C})\left(\right.$ resp. $\left.P^{1}(\mathbb{C})\right)$. The complement in $K^{4}$ of the $P^{1}(\mathbb{C})$ corresponding to the horizontal motions is diffeomorphic to the complement of $P^{1}(\mathbb{C})$ in $P^{2}(\mathbb{C})$, that is $\mathbb{C}^{2}=\mathbb{R}^{4}$, and the mapping

$$
D_{\epsilon}=\left\{\left(u_{1}, u_{2}\right) \in \mathbb{C}^{2},\left|u_{1}\right|^{2}+\left|u_{2}\right|^{2}<\epsilon^{2}\right\} \ni\left(u_{1}, u_{2}\right) \mapsto\left(u_{1}, u_{2}, z_{1}, z_{2}\right) \in J^{5}
$$

uniquely defined by the conditions that $z_{1}$ et $z_{2}$ be real and $z_{1}>0$ gives in the quotient a symplectic diffeomorphism of $D_{\epsilon}$ endowed with the sympectic form $d u_{1} \wedge d \bar{u}_{1}+d u_{2} \wedge d \bar{u}_{2}$ onto $K^{4} \backslash P^{1}(\mathbb{C})$ endowed with the symplectic form coming from the reduction. Writing the secular Hamiltonian in such coordinates amounts to performing Jacobi's reduction of the node: geometrically, it corresponds to the identity $\theta_{2}-\theta_{1}=\pi$, which is satisfied when the angular momentum is vertical (compare Figure 2). This identity implies the disappearance of the angles $\theta_{1}$ et $\theta_{2}$ from the expression of the Hamiltonian which, because of its invariance under rotations around the vertical axis, depends only on their difference.

\subsection{Quadratic part and singularities}

Once the angular momentum has been fixed in the vertical direction, the secular Hamiltonian admits two symmetries in addition to the rotations around the vertical axis:

1) the symmetry with respect to a vertical plane, for example the plane $x_{1} 0 x_{3}$, which acts on the longitudes by

$$
\left(g_{1}, g_{2}, \theta_{1}, \theta_{2}\right) \mapsto\left(\pi-g_{1}, \pi-g_{2}, \pi-\theta_{1}, \pi-\theta_{2}\right),
$$

and becomes in the $u, z$ coordinates

$$
\left(u_{1}, u_{2}, z_{1}, z_{2}\right) \mapsto\left(\bar{u}_{1}, \bar{u}_{2},-\bar{z}_{1},-\bar{z}_{2}\right) ;
$$

2 ) the rotation by $\pi$ around the common node, which acts on the longitudes by

$$
\left(g_{1}, g_{2}, \theta_{1}, \theta_{2}\right) \mapsto\left(-g_{1},-g_{2}, \theta_{1}, \theta_{2}\right)
$$


and becomes, after composition with the rotation by $-2 \theta_{1}$ around the vertical axis,

$$
\left(u_{1}, u_{2}, z_{1}, z_{2}\right) \mapsto\left(\bar{u}_{1}, \bar{u}_{2}, \bar{z}_{1}, \bar{z}_{2}\right) .
$$

Composing both and then rotating by $\pi$ around the vertical axis, we get that the secular Hamiltonian is invariant by complex conjugation and by the transformation

$$
\left(u_{1}, u_{2}, z_{1}, z_{2}\right) \mapsto\left(-u_{1},-u_{2}, z_{1}, z_{2}\right)
$$

It follows that the quadratic part of the Taylor expansion of the secular Hamiltonian at its "circular horizontal" singularity has the form

$$
a_{1}\left|u_{1}\right|^{2}+a_{2}\left|u_{2}\right|^{2}+b \operatorname{Re}\left(u_{1} \bar{u}_{2}\right)+c_{1}\left|z_{1}\right|^{2}+c_{2}\left|z_{2}\right|^{2}+d \operatorname{Re}\left(z_{1} \bar{z}_{2}\right),
$$

which one writes classically

$$
\begin{aligned}
Q\left(\xi_{1}, \xi_{2}, \eta_{1}, \eta_{2}, p_{1}, p_{2}, q_{1}, q_{2}\right) & =Q^{\prime}\left(\xi_{1}, \xi_{2}\right)+Q^{\prime}\left(\eta_{1}, \eta_{2}\right) \\
& -Q^{\prime \prime}\left(p_{1}, p_{2}\right)-Q^{\prime \prime}\left(q_{1}, q_{2}\right),
\end{aligned}
$$

where $Q^{\prime}$ et $Q^{\prime \prime}$ are real quadratic forms of two variables known since the 18th century (see [Ti, Cha]):

$$
\left\{\begin{array}{l}
Q^{\prime}(X, Y)=m_{1} m_{2}\left\{\frac{1}{8} B^{(1)}\left(\frac{X^{2}}{\Lambda_{1}}+\frac{Y^{2}}{\Lambda_{2}}\right)-\frac{1}{4} B^{(2)} \frac{X Y}{\sqrt{\Lambda_{1} \Lambda_{2}}}\right\}, \\
Q^{\prime \prime}(X, Y)=m_{1} m_{2}\left\{\frac{1}{8} B^{(1)}\left(\frac{X}{\sqrt{\Lambda_{1}}}-\frac{Y}{\sqrt{\Lambda_{2}}}\right)^{2}\right\}
\end{array}\right.
$$

where the Laplace coefficients $B^{(1)}$ and $B^{(1)}$ depend only on $\Lambda_{1}, \Lambda_{2}$. Keeping only the quadratic terms in the eccentricities $e_{1}, e_{2}$ and the inclinations $i_{1}, i_{2}$, and taking into account that $\theta_{2}-\theta_{1}=\pi$ (see 2.3), one finds that the dominant terms of the secular Hamiltonian are 30 .

$$
m_{1} m_{2}\left[\frac{1}{8} B^{(1)}\left[e_{1}^{2}+e_{2}^{2}-\left(i_{1}+i_{2}\right)^{2}\right]+\frac{1}{4} B^{(2)} e_{1} e_{2} \cos \left(g_{1}-g_{2}\right)\right] \text {. }
$$

Finally, replacing the two equations which fix the direction of the angular momentum by their linearization ${ }^{31} z_{1} \sqrt{2 \Lambda_{1}}+z_{2} \sqrt{2 \Lambda_{2}}=0$, and choosing in the subspace (diffeomorphic to $\mathbb{C}^{3}=\mathbb{R}^{6}$ ) which they define the coordinates $u_{1}, u_{2}, z_{1}$, the restriction of $Q$ becomes the quadratic form

$$
\left\{\begin{array}{r}
Q_{0}=Q^{\prime}\left(\xi_{1}, \xi_{2}\right)+Q^{\prime}\left(\eta_{1}, \eta_{2}\right)-m_{1} m_{2} \frac{B^{(1)} \Lambda_{1}}{8}\left(\frac{1}{\Lambda_{1}}+\frac{1}{\Lambda_{2}}\right)^{2}\left(p_{1}^{2}+q_{1}^{2}\right) \\
=\frac{m_{1} m_{2}}{8}\left\{B^{(1)}\left(\frac{\left|u_{1}\right|^{2}}{\Lambda_{1}}+\frac{\left|u_{2}\right|^{2}}{\Lambda_{2}}\right)-2 B^{(2)} \frac{\operatorname{Re}\left(u_{1} \bar{u}_{2}\right)}{\sqrt{\Lambda_{1} \Lambda_{2}}}\right\} \\
-\frac{1}{8} m_{1} m_{2} B^{(1)} \Lambda_{1}\left(\frac{1}{\Lambda_{1}}+\frac{1}{\Lambda_{2}}\right)^{2}\left|z_{1}\right|^{2} .
\end{array}\right.
$$

\footnotetext{
${ }^{30}$ There are sign discrepancies with the formula that Poincaré attributes to Tisserand in section 48; in particular, having different conventions for inclinations, his formula for mutual inclination is $i_{1}-i_{2}$ instead of $i_{1}+i_{2}$.

${ }^{31}$ These linearized equations are obviously first integrals of the Hamiltonian system defined by $Q$.
} 
It remains to understand the topology of the level hypersurfaces of the restriction of $Q_{0}$ to the ellipsoid defined by the dominant term of the equation fixing the norm of the angular momentum (supposed to be close to its maximal value corresponding to circular horizontal motions), that is

$$
\left|u_{1}\right|^{2}+\left|u_{2}\right|^{2}+\frac{\Lambda_{1}+\Lambda_{2}}{\Lambda_{2}}\left|z_{1}\right|^{2}=\epsilon^{2}
$$

If $\epsilon$ is small, the topology will be the same as for the reduced secular Hamiltonian itself. There are exactly three critical points, corresponding to the eigenplanes of $Q_{0}{ }^{32}$ two of them correspond to horizontal ellipses whose perihelia are in conjunction $\left(\Sigma^{\rightarrow}\right)$ or opposition $\left(\Sigma^{\leftrightarrow}\right)$; the third one $\Sigma^{\odot}\left(u_{1}=u_{2}=0\right)$ corresponds to a pair of circles with non-zero inclination (see figure 4). As singular points of the reduced secular Hamiltonian, $\Sigma^{\odot}$ and $\Sigma^{\leftrightarrow}$ are elliptic while $\Sigma^{\rightarrow}$ is hyperbolic. But as singularities of the Hamiltonian vector field in $K^{4}$, the three singular points are elliptic (i.e., the eigenvalues of their linearized part are imaginary).

Remark. For the planar problem, the averaged system is completely integrable. Indeed, it has two degrees of freedom and two commuting integrals, the energy and the angular momentum. For a global study and the application to the existence of verious type of quasi-periodic solutions of the planar Three-Body Problem, see [Fe1].

\section{Periodic solutions 1) Local existence by continuation}

\subsection{Pénétrer dans une place jusqu'ici réputée inabordable}

The periodic solutions are the subject, in 1884, of the first work of Poincaré on the Three-Body Problem ([P4], announced by a note in the "Compte Rendus de l'Académie des Sciences" in 1883). They appear in section 36 of Chapter III of The New Methods. As a good astronomer, Poincaré called a solution periodic if it becomes such in some rotating frame, as is, in the first approximation, the case of the motion of the Moon seen from the Earth. In other words, a solution $x(t)$ is periodic in this sense if there exists a period $T$ such that for all $t, x(t+T)=\mathcal{R} x(t)$, where $\mathcal{R}$ is a rotation, that is if it is a periodic solution of the system reduced by the rotational symmetry.

These solutions play a key role in the whole work, and their study by continuation methods prefigures aspects of the theory of singularities:

Le problème que nous allons traiter ici est le suivant : Supposons que, dans les équations (1) ${ }^{33}$ les fonctions $X_{i}$ dépendent d'un certain paramètre $\mu$; supposons que dans le cas de $\mu=0$ on ait pu intégrer les équations, et qu'on ait reconnu ainsi l'existence d'un certain nombre de solutions périodiques. Dans quelles conditions aura-t-on le droit d'en conclure que les équations comportent encore des solutions périodiques pour les petites valeurs de $\mu$ ?34

\footnotetext{
${ }^{32}$ Because of the symmetries, they are the same for the Hamiltonian and for its quadratic part $Q_{0}$.

${ }^{33}$ These are the equations $d x_{i} / d t=X_{i}\left(x_{1}, \cdots, x_{n}\right), ; i=1, \cdots, n$.

${ }^{34}$ The problem we are going to address is the following: suppose that, in equations (1), the functions $X_{i}$ depend on some parameter $\mu$; suppose that, when $\mu=0$ one has integrated the equations and recognized the existence of some periodic solutions. Under what conditions shall we be able to conclude that for small values of $\mu$ the equations still possess periodic solutions?
} 
Annoncing the existence of an infinity of periodic solutions of the planar planetary Three-Body Problem when the planetary masses are sufficiently small, Poincaré justified his efforts as follows:

Il semble d'abord que ce fait ne puisse être d'aucun intérêt pour la pratique. En effet, il y a une probabilité nulle pour que les conditions initiales du mouvement soient précisément celles qui correspondent à une solution périodique. Mais il peut arriver qu'elles en diffèrent très peu, et cela a lieu justement dans les cas où les méthodes anciennes ne sont plus applicables. On peut alors avec avantage prendre la solution périodique comme première approximation, comme orbite intermédiaire, pour employer le langage de M. Gyldén.

Il y a même plus : voici un fait que je n'ai pu démontrer rigoureusement, mais qui me paraît pourtant très vraisemblable.

Étant données des équations de la forme définie dans le numéro 13 et une solution particulière quelconque de ces équations, on peut toujours trouver une solution périodique (dont la période peut, il est vrai, être très longue), telle que la différence entre les deux solutions soit aussi petite qu'on le veut, pendant un temps aussi long qu'on le veut. D'ailleurs, ce qui nous rend ces solutions périodiques si précieuses, c'est qu'elles sont, pour ainsi dire, la seule brèche par où nous puissions essayer de pénétrer dans une place jusqu'ici réputée inabordable 35

Then, describing Hill's researches on the Moon, he further explains the importance of the construction of approximating periodic solutions:

Supposons que, dans le mouvement d'un astre quelconque, il se présente une inégalit $\oint^{36}$ très considérable. Il pourra se faire que le mouvement véritable de cet astre diffère fort peu de celui d'un astre idéal dont l'orbite correspondrait à une solution périodique.

Il arrivera alors assez souvent que l'inégalité considérable dont nous venons de parler aura sensiblement le même coefficient pour l'astre réel et pour cet astre idéal ; mais ce coefficient pourra se calculer beaucoup plus facilement pour l'astre idéal dont le mouvement est plus simple et l'orbite périodique.

C'est à M. Hill que nous devons la première application de ce principe. Dans sa théorie de la Lune, il remplace ce satellite dans une première approximation par une Lune idéale, dont l'orbite est périodique. Le mouvement de cette Lune idéale est alors celui qui a été décrit au $n^{\circ} 41$, où nous avons parlé de ce cas particulier des solutions périodiques de la première sorte, dont nous devons la connaissance à M. Hill.

\footnotetext{
${ }^{35}$ It seems at first that this fact cannot be of any interest in practice. Indeed, there is zero probability that the initial conditions of motion be precisely those which correspond to a periodic solution. But it may happen that they differ very little, and this occurs precisely where the old methods no longer apply. We can then use the periodic solution as a first approximation, as an intermediate orbit, to use the language of Mr. Gyldén. There is even more: here is a fact that I could not prove rigorously, but which nevertheless seems very likely to me. Given equations of the form defined in $\mathrm{n}^{o} 13$ and an arbitrary solution of these equations, one can always find a periodic solution (with a period which, admitedly, may be very long), such that the difference between the two solutions be arbitrarily small. In fact, what makes these solutions so precious to us, is that they are, so to say, the only opening through which we can try to enter a place which, up to now, was deemed inaccessible.

36 that is, a deviation from the elliptic motion due to the action of the Sun.
} 
Il arrive alors que le mouvement de cette Lune idéale, comme celui de la Lune réelle, est affecté d'une inégalité considérable bien connue sous le nom de variation ; le coefficient est à peu près le même pour les deux Lunes. M. Hill calcule sa valeur pour sa Lune idéale avec un grand nombre de décimales. Il faudrait, pour passer au cas de la nature, corriger le coefficient ainsi obtenu en tenant compte des eccentricités, de l'inclinaison et de la parallaxe. C'est ce que M. Hill eût sans doute fait s'il avait achevé la publication de son admirable Mémoire ${ }^{37}$

Moreover, we shall see that periodic solutions play a key role in the proof of nonintegrability of the Three-Body Problem. The various objects attached to them are of common use in dynamics: the characteristic exponents are introduced in Chapter IV, the asymptotic solution $\$^{38}$ in Chapter VII, the doubly asymptotic solution ${ }^{39}$ in Chapter XXXIII.

\subsection{The three "sorts"}

In section 39, Poincaré recalls the classification of periodic solutions into three sort:40 from his 1884 paper:

... j'ai été conduit à distinguer trois sortes de solutions périodiques: pour celles de la première sorte, les inclinaisons sont nulles et les eccentricités très petites; pour celles de la deuxième sorte, les inclinaisons sont nulles et les eccentricités finies ; enfin, pour celles de la troisième sorte, les inclinaisons ne sont plus nulles 41

These three sorts are continuations of periodic solutions which exist for the limit problem $F_{0}(x)$ which consists in two uncoupled Kepler Problems. Namely

1) two circular motions in the same plane;

2) two elliptic motions in the same plane with resonant frequencies, perihelia in conjunction or opposition and initial mean longitudes both equal to zero;

3) two circular motions in different planes with resonant frequencies.

In the first sort, the eccentricities of the planets are small and they have no inclination (planar problem); in the limit where the masses vanish, the orbits become circular, which is the singularity of the secular Hamiltonian: if $n_{1}, n_{2}$ are the frequencies of

\footnotetext{
${ }^{37}$ Suppose that, in the motion of some star, there is a very strong inequality. It may happen that the true motion of this star will differ very little from the motion of an ideal star whose orbit would correspond to a periodic solution. It will often happen that the large inequality which we just mentioned will have approximately the same coefficient for the real star and for this ideal star; but this coefficient will be much more easily computed for the ideal star whose motion is simpler and the orbit is periodic. It is to Mr Hill that we owe the first application of this principle. In his Lunar theory, he replaces this satellite in the first approximation by an ideal Moon, whose orbit is periodic. The motion of this ideal Moon is then the one described in section $\mathrm{n}^{\circ} 41$, where we discussed of this special case of periodic solutions of the first sort, which we know thanks to Mr Hill. It then arrives that the motion of this ideal Moon is affected of a very large inequality, well known under the name of variation; the coefficient is approximately the same for the two Moons. Then Mr Hill computes its value for his ideal Moon with a large number of decimals. In order to treat the case of nature, one would need to correct the coefficient obtained in this way by taking into account the eccentricities, the inclination and the parallax. This is most probably what Mr Hill would have done if he had completed the publication of his admirable Memoir.

${ }^{38}$ Today called stable or unstable manifolds, or more generally invariant manifolds.

${ }^{39}$ Today called homoclinic or heteroclinic solutions.

40 in French: les trois sortes.

${ }^{41}$ I was led to distinguish three sorts of periodic solutions: for those of the first sort, the inclinations are equal to zero and the eccentricities are very small, for those of the second sort, the inclinations are equal to zero and the eccentricities are finite; finally, for those of the third sort, the inclinations are no longer equal to zero.
} 
the two circular Keplerian motions, the limit solution has period $T=\frac{2 \pi}{n_{1}-n_{2}}$ : indeed after time $T$ the two bodies have turned respectively by the angles $\frac{2 \pi n_{1}}{n_{1}-n_{2}}$ and $\frac{2 \pi n_{2}}{n_{1}-n_{2}}$ whose difference is $2 \pi$. In terms of the coordinates introduced in 3.1, the existence proof for $\mu \neq 0$ amounts to writing that the expressions $\Lambda_{1}, \Lambda_{2}, u_{1} e^{i \lambda_{1}}, u_{2} e^{i \lambda_{2}}, \lambda_{1}-\lambda_{2}$ take the same values at times 0 and $T$, which expresses that after time $T$ the mutual distances between the bodies have regained their initial value 42 . Writing these as functions of the initial conditions and the period and taking into account the first integrals, Poincaré shows that the continuation of such solutions to small values of $\mu$ is possible provided $n_{1}$ is not an integer multiple of $n_{2}-n_{1}$ (this ensures that the ad hoc Jacobian determinant does not vanish). He also notices that the periodic solutions obtained in this way have necessarily the following property: the bodies are in symmetricat ${ }^{43}$ conjunction. at some time $t_{0}$, and in symmetrical opposition at time $t_{0}+T / 2$.

The most famous example of a periodic solution of the first sort (in the case of the Restricted Problem, that is when one of the masses is infinitesimal, see 9.1) is Hill's intermediate orbit used in his study of the Lunar problem. Describing Hill's result in par. 41, Poincaré corrects a wrong guess of Hill about the global continuation of his solution (see 4.3); see the answer sent by Hill to Poincaré at the address http://www .univ-nancy2.fr/poincare/chp/text/hill18920120.xml.

In the second sort, the inclinations are still zero but the eccentricities are finite; in the limit one gets elliptic motions with the same direction of semi-major axes and conjunctions or oppositions at each half-period. In the third sort, eccentricities are small but inclinations are finite and the limit motions are circular but inclined. Here we are facing a bifurcation problem: indeed, we start from the degenerate and completely integrable situation where families of periodic solutions of the reduced problem which exist for $\mu=0$ are expected to break for $\mu \neq 0$ and give rise to isolated periodic solutions. More precisely, we start from a resonant torus of dimension 2, corresponding to motions along two Keplerian ellipses of the form $l_{1}(t)=n_{1} t+l_{1}^{0}, l_{2}(t)=n_{2} t+l_{2}^{0}$, where the values of $L_{1}$ and $L_{2}$ are chosen so that there exists $T \in \mathbb{R}$ and $k_{1}, k_{2} \in \mathbb{N}$ with $n_{1} T=k_{1} 2 \pi, n_{2} T=k_{2} 2 \pi$.

In what he called "Examen d'un important cas d'exception" "44, Poincaré shows that, when as in the (reduced) planetary problem, the unperturbed Hamiltonian $F_{0}$ depends only on some of the action variables 45 , that is when the phase variables are grouped into two subsets, the fast (or Keplerian) ones and the slow (or secular) ones, the continuation for $\mu \neq 0$ of the unperturbed periodic orbits by periodic orbits of the same period $T$, amounts to finding critical points, either non degenerate or of odd multiplicity (in particular maximum of minimum) of the average $R$ of the perturbing function $F_{1}$ along the unperturbed periodic solutions.

In the planar problem, $R$ depends on the 4 initial values $H^{0}, l_{1}^{0}, l_{2}^{0}, h^{0}$ of the reduced coordinates (see 2.4) (those of $L_{1}, L_{2}$ have already been fixed in order to fix the

\footnotetext{
${ }^{42}$ This is because $u e^{i \lambda}=\sqrt{2(L-G)} e^{i l}$; hence the given functions determine $a_{1}, a_{2}, e_{1}, e_{2}$ (that is the shape of both ellipses), $l_{1}, l_{2}$ (that is the position of the bodies on the ellipses) and $g_{1}-g_{2}$ (that is the relative position of the ellipses).

43 "Symmetrical" means that the velocities are othogonal to the line joining the bodies; this implies a symmetry with respect to this line of the motions of the bodies at times symmetrical with respect to $t_{0}$

${ }^{44}$ Consideration of an important exceptional case.

${ }^{45}$ In this case the Hessian of $F_{0}$ with respect to the action variables vanishes identically, which forbids using the trick of replacing $F_{0}$ by a function $\varphi\left(F_{0}\right)$ of $F_{0}$ whose Hessian does not vanish; introduced by Poincaré this trick allows in some cases (see for instance 9.1 to regain the possibility of using directly the implicit function theorem.
} 
unperturbed frequencies). Moreover, one can fix the phase, that is the origin of time, by setting $l_{1}^{0}=0$, which leaves the three variables $H^{0}, l_{2}^{0}, h^{0}$. In the spatial case, it depends on the 6 initial values of the reduced coordinates which can be reduced to the five $G_{1}^{0}, G_{2}^{0}, l_{2}^{0}, g_{1}^{0}, g_{2}^{0}$.

At this point, Poincaré notices that, provided one makes the ansatz that at some time (chosen to be $t=0$ ) the bodies are in symmetric conjunction, that is if in addition to $l_{1}^{0}$ we suppose that $l_{2}^{0}=g_{1}^{0}=g_{2}^{0}=0$, the dominant term of the expansion in eccentricities of $R$ is nothing but the reduced secular Hamiltonian ${ }^{46}$. Hence he is able to relate the existence of periodic solutions of the second and third sort to the singularities of the reduced secular system. He then appeals to the computations of the quadratic part of the averaged Hamiltonian done by the "founders of Celestial Mechanics" as they are reproduced in Tisserand's treatise ${ }^{47}$ [Ti] (see 3.3) to compute the critical points of the reduced Hamiltonian. This allows him in particular to show the existence of periodic solutions of the third sort different from those of the second sort, i.e., with non zero inclinations. In his characteristic style, he concludes:

Cela ne veut pas dire qu'il n'existe pas également des solutions périodiques de la troisième sorte pour lesquelles il n'y ait pas de conjonction symétrique ; il se pourrait en effet que, la fonction $R$ admît d'autres maxima ou minima que ceux qui correspondent au cas de $l_{0}^{\prime}=g_{0}=g_{0}^{\prime}=0$. Il y aurait donc lieu de revenir sur cette question ${ }^{48}$

Notice that this ansatz is crucial to make precise the link with the singularities of the secular system; indeed, because of the assumed resonance between the mean motions of the planets, the secular system is not a good approximation to the actual system.

Figure 4 is an attempt to represent the unperturbed periodic solutions from which the second and third sort periodic solutions bifurcate: the 8-dimensional reduced phase space of the spatial Three-Body Problem is fibered over the 4-dimensional reduced secular space $K^{4}$, diffeomorphic to the complex projective plane $P_{2}(\mathbb{C})$. In the representation, I have replaced $K^{4}$ by its "real part", diffeomorphic to the real projective plane $P_{2}(\mathbb{R})$, obtained by gluing along their boundary (diffeomorphic to a circle) a Moebius band and a disc. This is not completely stupid as, because of the symmetries of the secular Hamiltonian (invariance under complex conjugacy, see 3.3), the critical points we are interested in are contained in this real part. The fibers of the projection on $K^{4}$ are diffeomorphic to $\mathbb{T}^{2} \times \mathbb{R}^{2}$. Once a resonant pair of values of the semi-major axes (more precisely a resonant pair $\left(\Lambda_{1}, \Lambda_{2}\right)$ ) has been fixed, the fibers become 2-tori, parametrized by the mean longitudes $\left(\lambda_{1}, \lambda_{2}\right)$ and each of these tori is filled by a family of periodic solutions. The ansatz amounts to choosing one (in fact two, corresponding to opposition of conjunction) of these periodic solutions. Among those, the ones lying over the critical points of the reduced

\footnotetext{
${ }^{46}$ More precisely, one must also avoid those mean motion resonances which give rise to periodic terms of order $\leq 2$ in eccentricities, the first case being the $(1,1)$-resonance, that is $n_{1}=n_{2}$, which contributes a periodic term whose coefficient is of order 0 in eccentricities.

${ }^{47}$ Mentioning this work in the introduction of the Lessons, Poincaré writes (see 6.4): I had not to do again what he had done and done well.

${ }^{48}$ This does not mean that solutions of the third sort for which there is no symmetric conjunction do not exist; it could indeed be the case that the function $R$ possesses other maxima or minima that the ones which correspond to the case of $l_{0}^{\prime}=g_{0}=g_{0}^{\prime}=0$. Hence one should come back to this question.
} 
secular system will be ones for wich Poincaré proves that they can be continued after the perturbation is turned on.

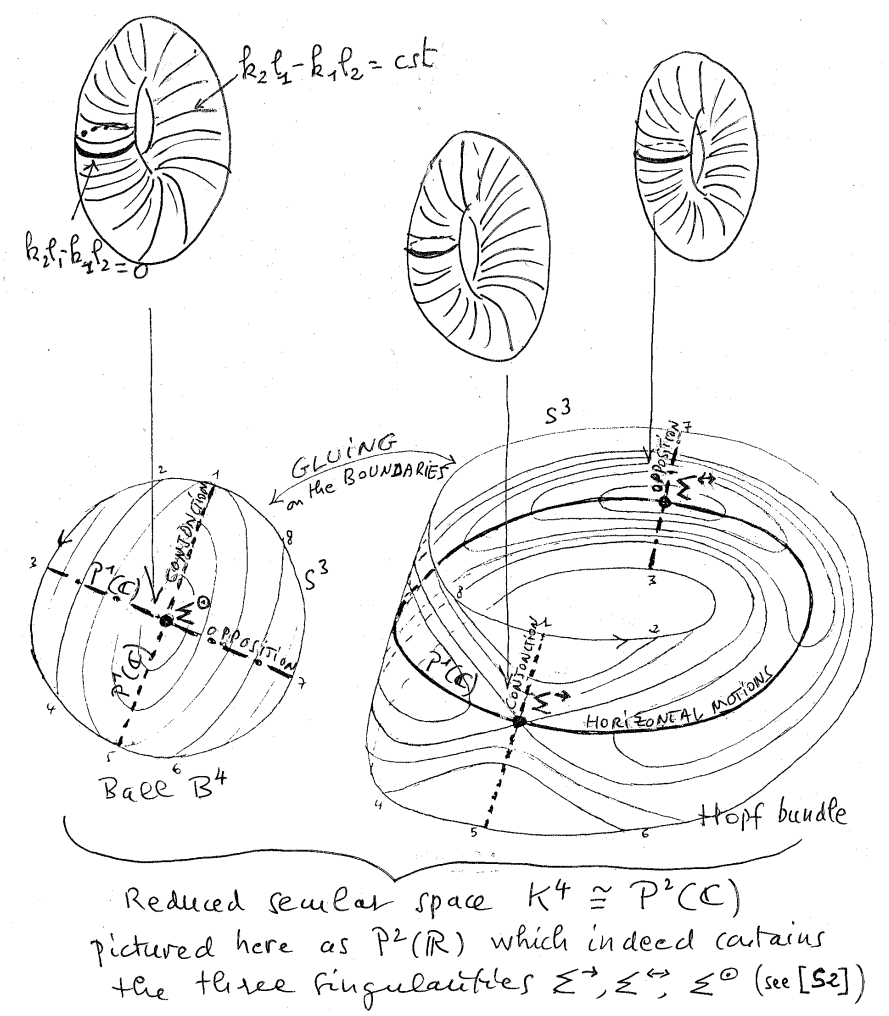

Figure 4: Unperturbed solutions at the origin of 2nd and 3rd sort orbits.

\section{3 and some others}

For example the ones in the neighborhood of an equilibrium position, mentioned in sections 51 and 52. This is one the few times Poincaré mentions a relative equilibrium, namely the Euler ones $L_{1}, L_{2}$ of the Restricted Problem (see Figure 12), where the Moon would stay at constant distance of the Earth and always be in opposition or conjonction. He shows the existence in the neighborhood of these equilibria of periodic solutions (all this of course in the rotating frame, see 91) which agree with Hill's intuition even if Hill's reasoning (continuation of his solution 4.2) was wrong.

\section{Quasi-periodic solutions 1) Formal aspects: Lindstedt series}

Il y a entre les géomètres et les astronomes une sorte de malentendu au sujet de la signification du mot convergence ${ }^{49}$

The first chapter of the second volume of The New Methods is devoted to the definition of what Poincaré calls an asymptotic series. It opens with a clarification of the different conceptions geometers and astronomers have of the notion of convergence, the astronomers paying essentially attention to the way the first terms decrease or increase (least term summation). Well aware of the practical side of the question, he specifies:

\footnotetext{
${ }^{49}$ There is between geometers and astronomers some sort of misunderstanding about the meaning of the word convergence.
} 
Les deux règles sont légitimes: la première, dans les recherches théoriques; la seconde, dans les applications numériques. Toutes deux doivent régner, mais dans deux domaines séparés et dont il importe de bien connaître les frontières.

Les astronomes ne les connaissent pas toujours d'une façon bien précise, mais ils les franchissent rarement; l'approximation dont ils se contentent les maintient d'ordinaire beaucoup en deçà; d'ailleurs leur instinct les guide et, s'il les trompait, le contrôle de l'observation les avertirait promptement de leur erreur 50

The excellent survey Ra1 by J.P. Ramis renders unnecessary for me to further discuss Poincaré's conceptions on divergent series. I shall just mention the introduction of the Lessons [P12], where Poincaré studies the "ancient methods" without paying attention to the problems of convergence. Alluding to The New Methods, he says:

D'autre part, dans ces Volumes, j'ai poussé l'approximation beaucoup plus loin que ne l'exige la pratique ; j'ai pu ainsi faire ressortir des circonstances tout à fait imprévues, dont l'importance analytique est très grande, mais qui n'ont aucun intérêt pout l'astronome praticien, et n'en acquerront que le jour où la précision des observations sera beaucoup plus grande qu'aujourd'hui, ou quand on voudra comparer des observations s'étendant sur une longue suite de siècles ${ }^{51}$

If one is content with replacing centuries by hundreds of millions of years and observations by numerical simulations, J. Laskar's works (see [Las1, Las2]) attest the relevance of this sentence.

The search for quasi-periodic solutions of the equations, while a purely mathematical problem (behaviour of the solutions in infinite time!) has nevertheless astronomical significance if one is interested in the behaviour on very long periods of time ${ }^{52}$ It is intimately linked with the method of averaging already discussed in section 3 . Indeed, averaging or (normal forms) and secular systems will appear naturally in what follows.

Paraphrasing Poincaré, I must say that my way of exposing these chapters will differ quite a lot from Poincaré's exposition but that the series I shall obtain are the same as his. Also, I shall not discuss the attributions: Newcomb? Lindstedt? Gyldén? Delaunay? Bohlin? Let us say Poincaré for simplicity, if not completely accurate, this does not seem to be outrageously wrong. The interested reader may look in particular at $[\mathrm{PC}]$ and at the transparencies of a conference by P. Nabonnand (see [Na]) on the correspondence between Lindstedt and Poincaré.

\footnotetext{
${ }^{50}$ The two rules are legitimate: the first, in theoretical researches; the second, in the numerical applications. Both must reign, but within two distinct domains the boundaries of which it is important to know well. Astronomers do not always know them very precisely, but they seldom cross them; the approximation they are satisfied with usually maintains them much further; besides, their instinct guides them and, if it did cheat them, the control by the observation would quickly warn them of their error.

${ }^{51}$ On the other hand, in these Volumes, I pushed the approximation much further than needed in practice; this allowed me to put forward quite unforeseen circomstances, the analytical importance of which is very high, but which do not have any interest for the practitionning astronomer, and will become of interest only when the precision of observations will be much higher than today, or when one will be willing to compare observations taking place along a long sequence of centuries.

${ }^{52}$ Because of velocities close to the velocity of light are involved, this is particularly relevant in the study of the motion of particles in an accelerator, the relevant period of time becoming relatively short; see [M4].
} 


\subsection{What is a Lindstedt series?}

The second volume of The New Methods of Celestial Mechanics is devoted to the the study of the perturbation series, the main tool used by astronomers in order to "solve" the equations of motion. Immediately after a chapter dedicated to the notion of asymptotic expansion, for the study of which I refer to [Ra1, Poincaré shows the existence of quasi-periodic "solutions" of equations (1), of the form

$$
\left\{\begin{array}{c}
x=x^{0}+\mu \Phi_{1}(w)+\mu^{2} \Phi_{2}(w)+\cdots, \\
y=w+\mu \Psi_{1}(w)+\mu^{2} \Psi_{2}(w)+\cdots,
\end{array}\right.
$$

where the $\Phi_{j}$ and the $\Psi_{j}$ are mappings from $\mathbb{T}^{N}$ to $\mathbb{R}^{N}$ and

$$
w=\left(w_{1}, w_{2}, \cdots, w_{N}\right), \quad w_{i}(t)=\bar{w}_{i}(\mu)+n_{i}(\mu) t .
$$

Such solutions look like what a family depending on $\mu$ of solutions (2) of a family of integrable systems (1) defined by $F_{0}(\chi, \mu)$ (the notations are those of 2.5 with $(x, y)$ replaced by $(\chi, w)$ and the parameter $\mu$ added) would become after a family of "coordinate changes" (see Figure 5):

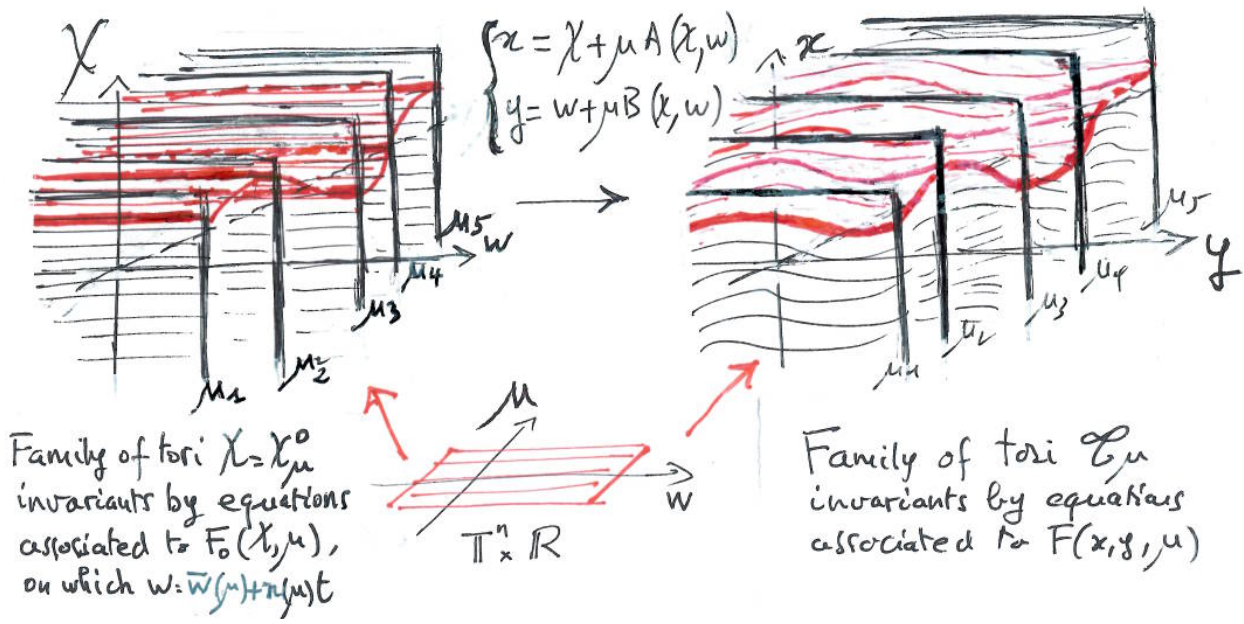

Figure 5: Families of invariant tori defined by Lindstedt series.

Using the implicit function theorem, one finds that the corresponding integral curves are confined for each $\mu$ to a torus $\mathcal{T}_{\mu}$ of the form

$$
x=x^{0}+\mu x^{1}(y)+\mu^{2} x^{2}(y)+\cdots
$$

invariant by the flow of the equations (1) defined by $F(x, y, \mu)$. In particular, they define motions in which the bodies stay forever in past and future at finite distance from each other without any collision ever happening. The gran of salt, which explains the brackets, is that the "solutions" and the "coordinate changes" are all given by formal series in $\mu$, the convergence of all of which would contradict Poincaré's non-integrability result. The novelty is that these series are devoid of the disturbing secular terms, containing powers of the time $t$ outside the sines and cosines, which, growing without limits, did spoil the series given by the "old methods" and appear now as mere artefacts of the Taylor expansion ${ }^{53}$. Poincaré calls these "solutions" Lindstedt series because the fist terms had been obtained by Lindstedt, but he is the first who proves their existence, as he himself explains :

\footnotetext{
${ }^{53}$ It is fair to quote d'Alembert who, in 1759, writes somewhat bitterly in volume 9 of the Encyclopédie [En] C6]
} 
Mais il y a une autre difficulté plus grave; on constate aisément que la méthode est applicable dans les premières approximations, mais on peut se demander si l'on ne sera pas arrêté dans les approximations suivantes; M. Lindstedt n'avait pu l'établir rigoureusement et conservait même à ce sujet quelques doutes. Ces doutes n'étaient pas fondés et sa belle méthode est toujours légitime; je l'ai démontré d'abord par l'emploi des invariants intégraux dans le Bulletin astronomique, t. III, p. 57, puis, sans me servir de ces invariants, dans les Comptes rendus, t. CVIII, p. 21. ${ }^{54}$

\subsection{Different types of Lindstedt series}

In order to understand the problems of convergence of the Lindstedt series, one needs to understand the choice one has when defining them. Consider again the case when

$$
F(x, \mu)=F_{0}(x)+\mu F_{1}(x)+\cdots
$$

does not depend on the angles $y$. For any value of $\mu$, the phase space $\mathbb{T}^{n} \times \mathbb{R}^{n}$ is foliated by invariant tori of the form $x=$ cst. A Lindstedt series is obtained in this case by chosing for each $\mu$ one of these invariant tori, depending nicely on $\mu$. This leads to three main types: series with variable frequencies, series with fixed frequencies, or series with fixed energy and fixed projective frequencies. All this is implicit in Poincaré who, nevertheless choses as does Lindstedt, the series with variable frequencies because they are those which are the most opposed to those with fixed frequencies which are given by the old methods. We shall write

$$
\omega(x, \mu)=\frac{\partial F}{\partial x}(x, \mu)=\omega^{0}(x)+\mu \omega^{1}(x) \cdots
$$

the frequency vector. If the initial conditions $(\bar{x}, \bar{y})=\left(x^{0}, y^{0}\right)$ are fixed independently of $\mu$, one obtains a family of quasi-periodic solutions of the family of equations $\left(H_{\mu}\right)$, whose frequencies $n(\mu)=\omega\left(x^{0}, \mu\right)$ depend in general of $\mu$. It is the most trivial example of a Lindstedt series.

If on the contrary one wants the frequencies to be fixed, that is independent of the parameter $\mu$, one must chose initial conditions $(\bar{x}(\mu), \bar{y}(\mu))$ satisfying the

\footnotetext{
“Je ne dois pas oublier d'ajouter $1^{\circ}$. que ma méthode pour déterminer le mouvement de l'apogée, est très-élégante \& très-simple, n'ayant besoin d'aucune intégration, $\&$ ne demandant que la simple inspection des coefficients du second terme de l'équation différentielle; $2^{\circ}$. que j'ai démontré le premier par une méthode rigoureuse, ce que personne n'avoit encore fait, E̛ n'a même fait jusqu'ici, que l'équation de l'orbite lunaire ne devoit point contenir d'arcs de cercle; si on ajoute à cela la maniere simple $\mathscr{G}$ facile dont je parviens à l'équation différentielle de l'orbite lunaire, sans avoir besoin pour cela, comme d'autres géometres, de transformations 8 d'intégrations multipliées ; $\mathcal{E}$ le détail que j'ai donné ci-dessus de mes travaux $\mathcal{E}$ de ceux des autres géometres, on conviendra, ce me semble, que j'ai eu plus de part à la théorie de la lune que certains mathématiciens n'avoient voulu le faire croire".

"I must not forget to add $1^{\circ}$. that my method for determining the motion of the lunar apgee, is very elegant and very simple, needing no integration, and requiring only the simple inspection of coefficients of the second term of the differential equation; $2^{\circ}$. that I was the first to prove by a rigorous method, something that no one had yet accomplished, \& that has not been accomplishd until now, that the lunar orbit equation need contain no arcs at all; if one adds to this the simple and easy way by which I obtain the differential equation of the Lunar orbit, without having, as other geometers, to use for that multiple transformations and integrations, and the detail given above of my works and of the works of the other geometers, I think one will agree that I have more contributed to the Lunar theory than some mathematicians had wanted people to believe."

${ }^{54}$ But there is another, more serious, difficulty; one checks easily that the method can be applied in the first approximations, but one can ask if one will not be stopped in the next approximations; M. Lindstedt had not been able to prove it and he was even somewhat doubting it. These doubts were not founded and his beautiful method is always legitimate; I first proved it in the Bulletin astronomique, t. III, p. 57, by using the integral invariants, then without using them in the Comptes rendus, t. CVIII, p. 21.
} 
equation

$$
\omega(\bar{x}(\mu), \mu)=\omega\left(x^{0}, 0\right)=\omega^{0}\left(x^{0}\right)=n^{0}, \quad \bar{x}(0)=x^{0} .
$$

The implicit function theorem grants the possibility of such a choice for small enough $\mu$ as long as the matrix

$$
\frac{\partial \omega}{\partial x}\left(x^{0}, 0\right)=\frac{\partial \omega^{0}}{\partial x}\left(x^{0}\right)=\frac{\partial^{2} F_{0}}{\partial x^{2}}\left(x^{0}, 0\right)
$$

is invertible (one says that the Hamiltonian $F_{0}$ is isochronically non degenerate at the point $\left.x^{0}\right)$.

Finally, if one wants the energy to be independent of $\mu$, one can only fix the frequencies up to a common scaling factor, i.e., projectively; it is enough to choose initial conditions satisfying

$$
\left\{\begin{array}{l}
\omega(\bar{x}(\mu), \mu)=\lambda(\mu) n^{0} \\
F(\bar{x}(\mu), \mu))=\text { constante. }
\end{array}\right.
$$

This is possible as soon as

$$
\left(\begin{array}{cc}
\frac{\partial^{2} F_{0}}{\partial x^{2}}\left(x^{0}\right) & \frac{\partial F_{0}}{\partial x}\left(x^{0}\right) \\
\operatorname{tr} \frac{\partial_{0}}{\partial x}\left(x^{0}\right) & 0
\end{array}\right)
$$

is invertible, that is as soon as, at the point $x^{0}$, the Hamiltonian $F_{0}$ is isoenergetically non-degenerate.

\subsection{From the old methods to the new ones and back}

Let us consider now a Hamiltonian $F(x, y, \mu)$ which is still completely integrable but without supposing that $(x, y)$ be action-angle coordinates for $F$. That is, we suppose that they come from action-angle coordinates $(\chi, w)$ through the symplectic change of coordinates

$$
x_{i}=\chi_{i}+\mu \cos w_{i}, \quad y_{i}=w_{i}, \quad i=1, \cdots, n,
$$

which transforms $F$ into

$$
C(\chi, w)=C_{0}(\chi)+\mu C_{1}(\chi)\left(\text { with necessarily } C_{0}=F_{0}\right),
$$

whose solutions are quasi-periodic, of the form

$$
\chi=\bar{\chi}, \quad w=\bar{w}+\varpi(\bar{\chi}, \mu) t=\bar{w}+\varpi^{0}(\bar{\chi}) t+\mu \varpi^{1}(\bar{\chi}) t .
$$

One obtains in this way the solutions of the Hamiltonian system $\left(H_{\mu}\right)$ associated with $F$ in the form of Lindstedt series of one of the three forms:

1) Series with variable frequencies $\varpi\left(x^{0}, \mu\right)=n^{0}+\mu n^{1}$ :

$$
x=x^{0}+\mu \cos \left(y^{0}+n^{0} t+\mu n^{1} t\right), \quad y=y^{0}+n^{0} t+\mu n^{1} t .
$$

2) Series with fixed frequencies $n^{0}=\varpi\left(x^{0}, 0\right)$ :

$$
x=x^{0}+\mu\left(\alpha_{1}+\cos \left(y^{0}+n^{0} t\right)\right)+\mu^{2} \alpha_{2}+\cdots, \quad y=y^{0}+n^{0} t .
$$

3) Series with fixed energy and fixed projective frequencies:

$$
x=x^{0}+\mu\left[\alpha_{1}+\cos \left(y^{0}+\lambda(\mu) n^{0} t\right)\right]+\mu^{2} \alpha_{2}+\cdots, \quad y=y^{0}+\lambda(\mu) n^{0} t .
$$


The use of the strange notations

$$
\bar{\chi}(\mu)=x^{0}+\mu \alpha_{1}+\mu^{2} \alpha_{2}+\cdots, \quad \bar{w}(\mu)=y^{0}
$$

for the initial conditions, is motivated by the choice of staying as close as possible to Poincaré's notations.

All these series are of the form

$x=\bar{\chi}(\mu)+\mu \cos \left(y^{0}+\varpi^{0}(\bar{\chi}(\mu)) t+\mu \varpi^{1}(\bar{\chi}(\mu)) t\right), \quad y=y^{0}+\varpi^{0}(\bar{\chi}(\mu)) t+\mu \varpi^{1}(\bar{\chi}(\mu)) t$.

If we expand them in series of powers of $\mu$, one obtains "old-fashioned" series

$$
\left\{\begin{aligned}
x= & x^{0}+\mu\left(\alpha_{1}+\cos \left(y^{0}+n^{0} t\right)\right) \\
& +\mu^{2}\left[\alpha_{1}-\left(\frac{\partial \varpi^{0}}{\partial x}\left(x^{0}\right) \cdot \alpha_{1}+n^{1}\right) t \sin \left(y^{0}+n^{0} t\right)\right]+O\left(\mu^{3}\right), \\
y= & y^{0}+n^{0} t+\mu\left(\frac{\partial \varpi^{0}}{\partial x}\left(x^{0}\right) \cdot \alpha_{1}+n^{1}\right) t+O\left(\mu^{2}\right)
\end{aligned}\right.
$$

which contain secular terms and are developed into the fixed frequencies $n^{0}=\varpi^{0}\left(x^{0}\right)$. Knowing that Lindstedt had originally tried to suppress these secular terms by letting the frequencies vary but maintaining fixed the initial conditions, one could think that it is the shift from fixed to variable frequencies which makes the difference between old and new methods but what we have just done shows that the same aim may be attained a priori via an infinite number of ways, and in particular by keeping expansions with fixed frequencies: it suffices to make the initial conditions depend on $\mu$ in a well chosen way. For example, the first step must eliminate the term

$$
\frac{\partial \varpi^{0}}{\partial x}\left(x^{0}\right) \cdot \alpha_{1}+n^{1}
$$

which is the term of order 1 in $\mu$ of $\varpi(\bar{\chi}(\mu), \mu)$.

Remark. The fact that the mapping $(\chi, w) \mapsto(x, y)$ is symplectic is easily checked directly but it is better to notice that it admits the generating function (another invention of Poincaré) $S(\chi, y)=\chi \cdot y+\mu \sum \sin y_{i}$, which means that

$$
x=\frac{\partial S}{\partial y}(\chi, y), \quad w=\frac{\partial S}{\partial \chi}((\chi, y), \quad \text { that is } \quad d S(\chi, y)=x \cdot d y+w \cdot d \chi,
$$

from which follows the identity $0=d^{2} S=d x \wedge d y-d \chi \wedge d w$.

\subsection{One torus or a foliation by tori? (Poincaré's ambiguities)}

In the above examples, each solution of $\left(H_{\mu}\right)$ is quasi-periodic of the form $\left(L_{\mu}\right)$. In the phase space $D \times \mathbb{R}^{n}$, the closure of the integral curve defined by a convergent Lindstedt serie is, when $\mu$ is fixed, a torus $\mathcal{T}_{\mu}$ whose dimension lies between 1 (periodic orbit) if there exists a non zero real number $T$ and integers $p_{i}$ such that for all $i$ one has $n_{i}(\mu) T=p_{i}$, and $n$ (quasi-periodic orbit of general type) if no resonance exists between the frequencies $n_{i}(\mu)$, that is is there is no relation of the form

$$
k \cdot n(\mu)=\sum_{i=1}^{n} k_{i} n_{i}(\mu)=0
$$


in which $k=\left(k_{1}, k_{2}, \cdots, k_{n}\right)$ are integers among which at least one is different from zero. Therefore, we are naturally led to look, as Poincaré did, not for formal particular solutions of equations $\left(H_{\mu}\right)$, but for formally invariant tori of these equations. Moreover, the structure of the solutions $\left(L_{\mu}\right)$ shows that, at the formal level, these tori should admit angular coordinates $\theta=\left(\theta_{1}, \cdots, \theta_{n}\right)$ such that the restriction of the equations becomes a constant vector field: in the non-resonant case where the closure of the integral curve $\left(L_{\mu}\right)$ has dimension $n$, such coordinates $\theta$ are obviously $\left(w_{1}, w_{2}, \cdots, w_{n}\right)$ which satisfy

$$
\frac{d w_{i}}{d t}=n_{i}(\mu), \quad i=1, \cdots, n .
$$

Together with the density in the torus of the integral curves of the flow, this last property is a major constraint on an invariant torus of a Hamiltonian system: indeed, we notice after Michael Herman that the form $\Omega$ induced on such a torus by the symplectic form

$$
d x \wedge d y=\sum_{i=1}^{n} d x_{i} \wedge d y_{i}
$$

which is invariant under the flow of equations $\left(H_{\mu}\right)$, is necessarily constant in the $\theta$ coordinates:

$$
\Omega=\sum_{i=1}^{n} \sum_{j=1}^{n} c_{i j} d \theta_{i} \wedge d \theta_{j} .
$$

But, the standard symplectic form $d x \wedge d y$ being the coboundary of the Liouville form $x \cdot d y=\sum_{i=1}^{n} x_{i} d y_{i}, \Omega$ itself is a coboundary and, being constant, it cannot but vanish identically. Finally, in the non-resonant case, we are looking for a formally invariant $n$-torus on which the form induced by the symplectic form vanishes. Today, such a torus is said to be Lagrangian. The very structure of $\left(L_{\mu}\right)$ shows that these tori must be looked for close to the tori $\left\{x^{0}\right\} \times \mathbb{T}^{n}$, that is as the graphs of a formal mapping $\Sigma_{\mu}$ from $\mathbb{T}^{n}$ to $D$. Being Lagrangian is then equivalent to the existence of a mapping $y \mapsto S_{\mu}(y)$ whose derivative is $\Sigma_{\mu}$ :

$$
\Sigma_{\mu}(y)=\frac{d S_{\mu}}{d y}(y)
$$

The classical Hamilton-Jacobi theory tells us that the only condition which $S_{\mu}$ must satisfy is to be a global solution of the Hamilton-Jacobi equation

$$
F\left(\frac{d S_{\mu}}{d y}(y), y, \mu\right)=c(\mu),
$$

where $c(\mu)$ is a constant (recall that morally, $\mu$ is fixed). This is a simple translation of the fact that such invariant Lagrangian submanifolds are nothing but geometric solutions of the Hamilton-Jacobi equation. Unfortunately in the absence of other hypotheses, a Lagrangian torus $\mathcal{T}_{\mu}$ which is the graph of the derivative $\Sigma_{\mu}$ of a global solution $S_{\mu}$ of the Hamilton-Jacobi equation, has no reason to possess coordinates such that the Hamilton equations $\left(H_{\mu}\right)$ become a constant vector field (i.e., a quasiperiodic one). This last property is only ensured in case the torus belongs to an $n$-parameter family $\chi=\left(\chi_{1}, \chi_{2}, \cdots, \chi_{n}\right)$ of such tori, defined by a complete solution 
$S_{\mu}(\chi, y)$ of the Hamilton-Jacobi equation:

$$
F\left(\frac{\partial S_{\mu}}{\partial y}(\chi, y), y, \mu\right)=C(\chi, \mu)
$$

This is because such a complete solution is the generating function of a symplectic diffeomorphism $\mathcal{D}_{\mu}$

$$
(\chi, w) \mapsto(x, y),
$$

implicitely defined by the identity

$$
d S_{\mu}=w \cdot d \chi+x \cdot d y
$$

and in the coordinates $(\chi, w)$ the Hamiltonian $F(x, y, \mu)$ takes the form $C(\chi, \mu)$ independent of the angles $w$, hence is completely integrable: Our torus being defined by $\chi=\bar{\chi}$ and parametrized by the angles $w$, the equations of motion read

$$
\frac{d w}{d t}=\frac{\partial C}{\partial \chi}(\bar{\chi}, \mu)
$$

Of course, the only thing which we used is the existence of the family for $\chi$ in an infinitesimal neighborhood of $\bar{\chi}$ : it is in fact enough to be able to take a derivative with respect to $\chi$ at the point $\bar{\chi}$. This remark will play a fondamental role in the next section.

On these points, Poincaré is slightly ambiguous: looking for a non-resonant torus, he makes it depend on parameters with respect to which he takes derivatives in order to find the frequencies. He does not care, at least explicitely, about the possibility of encountering denominators equal to zero in the coefficients of the series which define the generating function ${ }^{55} S_{\mu}(\chi, y)$. Nevertheless, one can make sense of all this thanks to Émile Borel who taught us how to take the derivative of a function defined on a Cantor set.

\subsection{Existence of the Lindstedt series in the non-degenerate case}

Warning! My presentation, though close to Poincaré's, differs in that I immediately address the individual convergence of the coefficients of the series, while in ChapterX, Poincaré treats them also as formal series, ${ }_{56}^{56}$ waiting Chapter XIII for all convergence questions.

We are looking for a "function" $S_{\mu}(\chi, y)=S(\chi, y, \mu)$ of the form

$$
S(\chi, y, \mu)=\chi \cdot y+\sum_{i=1}^{\infty} \mu^{i} S_{i}(\chi, y)
$$

whose partial derivatives $\frac{\partial S_{i}}{\partial \chi}$ et $\frac{\partial S_{i}}{\partial y}$ are $2 \pi$-periodic in the variables $y_{j}$, and which satisfies formally an equation of the form

$$
F\left(\frac{\partial S}{\partial y}(\chi, y, \mu), y, \mu\right)=C(\chi, \mu)=C_{0}(\chi)+\sum_{i=1}^{\infty} \mu^{i} C_{i}(\chi)
$$

\footnotetext{
${ }^{55}$ This is because, contrarily to what we do in the next section, he works with formal series whose coefficients themselves are formal series coming from the multiplication of formal series.

${ }^{56}$ which he multiplies as formal series with formal series coefficients ...
} 
The choice as terms of order 0 terms in $\mu$ of $\chi \cdot y$, that is of a generating function of the Identity, is dictated by the fact that, as $F(x, y, 0)$ is independent of the angles $y$, there is no need to modify this first term. Note that the word "function" must be taken with a gran of salt as we deal with formal expressions for which no domain of definition is given. Our goal is indeed to give all this a meaning.

The periodicity constraints imply that, up to an arbitrary function of $\chi$ (corresponding to a harmless angular phase shift), the fuction $S_{i}$ may be written

$$
S_{i}(\chi, y)=\alpha_{i} \cdot y+s_{i}(\chi, y),
$$

where $\alpha_{i}=\left(\alpha_{i, 1}, \cdots, \alpha_{i, n}\right)$ belongs to $\mathbb{R}^{n}$, and $s_{i}$ is $2 \pi$-periodic with mean value zero in the variables $y_{j}$.

The identification of powers of $\mu$ in the series expansion of $\left(H J_{\mu}\right)$ gives then

$$
\omega^{0}(\chi) \cdot \frac{\partial S_{1}}{\partial y}(\chi, y)+F_{1}(\chi, y)=C_{1}(\chi), \quad\left(E_{1}(\chi)\right)
$$

where we have used the notation

$$
\omega^{0}(x)=\frac{\partial F_{0}}{\partial x}(x)
$$

for the frequency vector of the unperturbed Hamiltonian $F_{0}(x)$.

The parameter $\chi=x^{0}$ being fixed, the determination of a particular invariant torus (depending on $\mu$ ) is possible as long as $x^{0}$ is sufficiently non resonant, that is as long as it belongs to one of the subsets $D_{K, \nu}$ of $D$ defined as follows:

$$
D_{K, \nu}=\left\{x^{0} \in D, \forall k \in \mathbb{Z}^{n}-\{0\},\left|k \cdot \omega^{0}\left(x^{0}\right)\right| \geq \frac{K}{|k|^{\nu}}\right\} .
$$

The Fourier series of $s_{1}\left(x^{0}, y\right)$,

$$
s_{1}\left(x^{0}, y\right)=\sum_{k=\left(k_{1}, \cdots k_{n}\right) \in \mathbb{Z}^{n}-\{0\}} \sigma_{k}\left(x^{0}\right) e^{i k \cdot y},
$$

is uniquely determined from that of $F_{1}\left(x^{0}, y\right)$,

$$
F_{1}\left(x^{0}, y\right)=\sum_{k=\left(k_{1}, \cdots k_{n}\right) \in \mathbb{Z}^{n}} f_{k}\left(x^{0}\right) e^{i k \cdot y},
$$

by the identities

$$
\sigma_{k}\left(x^{0}\right)=\frac{f_{k}\left(x^{0}\right)}{i k \cdot \omega^{0}\left(x^{0}\right)} .
$$

It is not difficult to see that, as the coefficients of the Fourier series of an analytic function decrease quicker than any power of $|k|$ and as the increase of the small denominators $i k \cdot \omega^{0}\left(x^{0}\right)$ is polynomial in $|k|$, the series so obtained defines an analytic function of $y$ as long as $x^{0}$ belongs to one the the $D_{K, \nu}$.

Concerning $\alpha_{1}$ and $C_{1}\left(x^{0}\right)$, they must be chosen in such a way as to satisfy

$$
C_{1}\left(x^{0}\right)-\omega^{0}\left(x^{0}\right) \cdot \alpha_{1}=\frac{1}{(2 \pi)^{n}} \int_{\mathbb{T}^{n}} F_{1}\left(x^{0}, y\right) d y,
$$

where the right-hand side is the averaged system, analogue in the non-degenerate case of the one studied in section 3.2 . 
One resolves in the same way, by induction, the equations which come from the identification of the terms in $\mu^{j}, j \geq 2$ in the series expansion of $\left(H J_{\mu}\right)$ : the identities are of the form

$$
\omega^{0}(\chi) \cdot \frac{\partial S_{j}}{\partial y}(\chi, y)+\Phi_{j}(\chi, y)=C_{j}(\chi)
$$

where the term $\Phi_{j}(\chi, y)$ is a polynomial in the $\frac{\partial S_{i}}{\partial y}(\chi, y), i<j$, whose coefficients are analytic in $\chi, y$.

Once chosen the $\alpha_{j}$, one obtains, for any $x^{0}$ belonging to one of the $D_{K, \nu}$, a formal series in $\mu, S\left(x^{0}, y, \mu\right)$, whose all coefficients are analytic functions of $y$, and a formal series in $\mu, C\left(x^{0}, \mu\right)$. The problem is now to "differentiate" these series with respect to $\chi_{1}, \cdots, \chi_{n}$.

Taking formally the derivative of the equation $\left(E_{j}(\chi)\right)$ with respect to the parameter $\chi_{k}$, one obtains new equations

$$
\omega^{0}(\chi) \cdot \frac{\partial^{2} S_{j}}{\partial \chi_{k} \partial y}(\chi, y)+\frac{\partial \omega^{0}}{\partial x}(\chi) \cdot \frac{\partial S_{j}}{\partial y}(\chi, y)+\frac{\partial \Phi_{j}}{\partial \chi_{k}}(\chi, y)=\frac{\partial C_{j}}{\partial \chi_{k}}(\chi),
$$

of the same form as above. Solving inductively these equations and the ones corresponding to higher derivatives, one determines, for each element $x^{0}$ of $D_{K, \nu}, S_{j}(\chi, y)$ and the $C_{j}(\chi)$ as formal series ${ }^{57}$ in $\left(\chi-x^{0}\right)$ (that is in the $\left(\chi_{i}-x_{i}^{0}\right), i=1, \cdots, n$ ) whose coefficients are analytical in $y$, which satisfy the equations $\left(E_{j}(\chi)\right)$.

As we already noticed, this is enough ${ }^{58}$ to ensure the quasi-periodicity of the motion on the formal Lagrangian torus defined by the equation $x=\frac{\partial S}{\partial y}\left(x^{0}, y, \mu\right)$. One deduces the existence of Lindstedt series, implicitely defined by

$$
\left\{\begin{array}{l}
x=x^{0}+\sum_{i=1}^{\infty} \mu^{i} \alpha_{i}+\sum_{i=1}^{\infty} \mu^{i} \frac{\partial s_{i}}{\partial y}\left(x^{0}, y\right) \\
y+\sum_{i=1}^{\infty} \mu^{i} \frac{s_{i}}{\chi}\left(x^{0}, y\right)=w=\bar{w}(\mu)+\frac{\partial C}{\partial \chi}\left(x^{0}, \mu\right) t
\end{array}\right.
$$

If, as Poincaré does, one choses to equal all the $\alpha_{i}$ to zero, one obtains Lindstedt series with variable frequencies. If, on the contrary, one choses the $\alpha_{i}$ in such a way ${ }^{59}$ that $\frac{\partial C_{i}}{\partial \chi}\left(x^{0}\right)$ vanishes for all $i \geq 1$, one obtains Lindstedt series with fixed frequencies.

Remarks. 1) In [Pos], Pöschel shows that the $S_{i}(\chi, y)$ (resp. the $\left.C_{i}(\chi)\right)$ are $C^{\infty}$ functions in the sense of Whitney on each closed subset $D_{K, \nu} \times T^{n}\left(\operatorname{resp} . D_{K, \nu}\right)$. This is equivalent to saying that they may be extended (in a non unique way) to $C^{\infty}$ functions on the whole of $D \times \mathbb{T}^{n}$ (resp. D). The first result if this type, for mappings, is due to V. Lazutkin [Laz].

2) At the end of section 147, Poincaré notices that the construction may be done without appealing to infinite sums at intermediary stages by truncating the Fourier series far enough to be entitled to putting the rest in the small perturbation:

${ }^{57}$ Notice that, for $j=1$, the two formal series in $\left(\chi-x^{0}\right)$,

$$
C_{1}(\chi)-\omega^{0}(\chi) \cdot \alpha_{1} \quad \text { and } \quad \frac{1}{(2 \pi)^{n}} \int_{\mathbb{T}^{n}} F_{1}(\chi, y) d y
$$

are identical. In particular, $C_{1}(\chi)$ is defined and analytic for all $\chi$.

${ }^{58}$ Obviously, order 1 is already enough.

${ }^{59}$ This is possible as soon as the unperturbed Hamiltonian $F_{0}$ is isochronically non-degenerate (see 5.2 . 
Voici donc ce qu'on sera conduit à faire: dans la série $F_{1}$, tous les termes, sauf un nombre fini d'entre eux, pourront être regardés comme du même ordre de grandeur que $\mu$; mais il y en aura qui seront du même ordre de grandeur que $\mu^{2}$, d'autres, plus petits encore, qui seront du même ordre de grandeur que $\mu^{3}$, etc. Dans les autres séries $F_{2}, F_{3}$, on trouvera de même des termes de ces divers ordres de grandeur.

Nous pourrons donc écrire en général

$$
F_{i}=F_{i, 0}+F_{i, 1}+F_{i, 2}+\cdots+F_{i, k} \cdots
$$

$F_{i, k}$ représentant ceux des termes de $F_{i}$ qui peuvent être regardés comme du même ordre de grandeur que $\mu^{k}$. Ces termes sont en nombre fini. Cette manière de décomposer $F_{i}$ comporte évidemment un assez grand degré d'arbitraire 60

This trick, which has the inconvenient of losing trace of the analyticity in the parameter, was used by Arnold in [A1, A3].

\subsection{Coping with the degeneracies ...}

At the end of section 128, Poincaré speculates that it is the degeneracy of the ThreeBody Problem which prevented Lindstedt from discovering the possibility of writing down series with fixed frequencies. And indeed such series cannot exist because, as the precessions of perihelia and nodes vanish with the perturbation, ${ }^{61}$ the slow frequencies must depend on the parameter $\mu$, i.e., one can only expect Lindstedt series whose frequencies are of the form $\left(\omega^{0}\left(x^{\prime 0}\right), \mu \omega^{1}\left(x^{0}\right)\right)$, where $x^{0}=\left(x^{\prime 0}, x^{\prime \prime 0}\right)$ is the decomposition of the action into fast and slow.

In order to show that Lindstedt series still exist in this case, Poincaré starts Chapter $\mathrm{X}$ by noticing that their existence in the non degenerate case applies to the averaged system in the neighborhood of circular and horizontal motions of the two planets:

On peut faire des principes du Chapitre précédent une application importante à l'étude de certaines équations que les astronomes ont souvent considérées 62

Indeed, after replacing the symplectic polar coordinates $H_{1}, H_{2}, Z_{1}, Z_{2}, h_{1}, h_{2}, \zeta_{1}, \zeta_{2}$ defined in 2.2 and 2.3 , by analogue coordinates which transform the quadratic part of the secular Hamiltonian into a sum of squares, and taking as a small parameter $\epsilon$ the distance to the origin (that is to zero eccentricities and inclinations), one can find a formal (in $\epsilon$ ) change of coordinates which eliminates the angles at any order provided the coefficients of the squares admit no relation with integer coefficients. Poincaré comments:

Ainsi on peut satisfaire formellement aux équations qui définissent les variations séculaires par des séries trigonométriques de la forme de MM. Newcomb et Lindstedt ... Ce résultat aurait été envisagé par Laplace ou Lagrange comme établissant complètement la stabilité du système solaire. Nous sommes plus difficiles aujourd'hui parce que la convergence

\footnotetext{
${ }^{60}$ The translation is an exercise for the reader.

${ }^{61}$ what Arnold calls a proper degeneracy.

${ }^{62}$ One can make of the principles of the former chapter an important application to the study of some equations that the astronomers have often considered.
} 
des développements n'est pas démontrée; le résultat n'en est pas moins important 63

This is nothing but putting the secular system into Birkhoff normal form (see [AKN]), that is finding a forma ${ }^{64}$ change of coordinates transforming the Poincaré coordinates $u_{j}=\xi_{j}+i \eta_{j}, z_{j}=p_{j}+i q_{j}$ into coordinates

$$
\tau_{j}+i \sigma_{j}=\sqrt{2 \rho_{j}} e^{i \omega_{j}}, j=1, \ldots, 4,
$$

such that the secular Hamiltonian depends only of their moduli and not on their arguments:

$$
H_{1}=\sum_{j=1}^{4} A_{j}\left(\tau_{j}^{2}+\sigma_{j}^{2}\right)+\sum_{1 \leq j, k,}^{4} B_{j, k}\left(\tau_{j}^{2}+\sigma_{j}^{2}\right)\left(\tau_{k}^{2}+\sigma_{k}^{2}\right)+\cdots
$$

The first step is transforming the quadratic part of the averaged system into a sum of squares by an orthogonal change of coordinates. Remembering the special form of the quadratic part (see 3.3) one sees that one can at this level make two independent linear changes of coordinates, one with the horizontal part (perihelia, eccentricities) to which correspond the horizontal frequencies $A_{1}$ and $A_{2}$, and the other with the vertical part (nodes and inclinations) (to which correspond the vertical frequencies $A_{3}$ and $\left.A_{4}\right)$.

Once the formal Birkhoff normal form is built (supposing there is no resonances between the $A j$ ), one eliminates the fast angles by a formal change of coordinates similar to the one used in the non degenerate case. This is possible because replacing $F_{0}$ by $F_{0}+\mu<F_{1}>$ (where $<F_{1}>$ is the result of averaging the fast angles in $F_{1}$ ) has removed the degeneracy. One could also have started by eliminating the fast angles independently of the form of the secular part, a process to which is attached today the name of Von Zeipel. In all cases, one has to stay outside a neighborhood of the set of values of the fast actions (here the semi-major axes) for which there is a mean motion resonance, in which case some combinations of the fast angles become slow and cannot be eliminated by averaging; this is the domain of Bohlin series (see 7), where resonant tori break into lower dimensional tori and their asymptotic manifolds.

We have in fact disregarded the difficulty of very small eccentricities and inclinations: indeed, the singular point of the secular system which corresponds to a pair of horizontal and circular ellipses gives rise in the full system to 2-dimensional invariant tori which after reduction become periodic solutions of the first sort (see 4.2). In order to be able to write Lindstedt series defining formal invariant Lagrangian tori very close to these (i.e., corresponding to quasi-periodic solutions whose eccentricities and inclinations are very small) it is necessary to center the symplectic polar coordinates one uses on these 2-dimensional tori. This is exactly what Poincaré does in his chapter XII.

\footnotetext{
${ }^{63}$ Hence one can formally satisfy the equations defining the secular variations by trigonometrical series similar to those of MM. Newcomb and Lindstedt ... This result would have been considered by Laplace or Lagrange as completely establishing the stability of the solar system. We are more demanding nowadays, because the convergence of these expansions is not proved; the result is nevertheless important.

${ }^{64}$ For the planar Three-Body Problem, the secular system can be reduced to a 1-degree of freedom system and hence is completely integrable; in this case, the procedure is not only formal.
} 

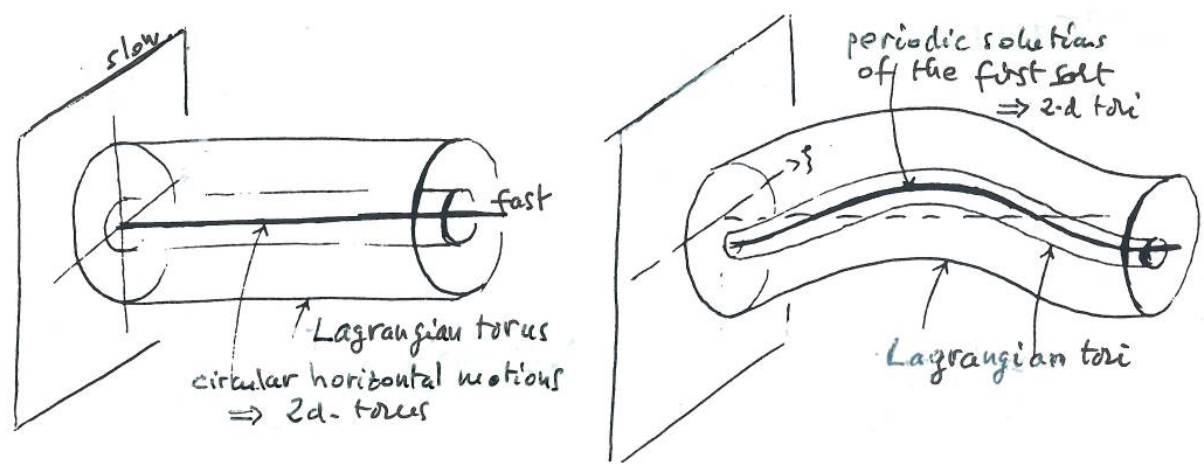

Figure 6: Invariant tori of very small eccentricities and inclinations.

Finally, if $x^{0}=\left(x^{\prime 0}, x^{\prime \prime 0}\right)$ belongs to a suitable subset of the actions, one obtains Lindstedt series whose frequencies are of the form

$$
\left(\omega^{0}\left(x^{\prime 0}\right), \mu \omega^{1}\left(x^{0}\right)\right) .
$$

This is as close as possible to the ones with fixed frequencies obtained in the non degenerate case.

\section{$5.7 \quad$... but forgetting a resonance!}

The method described in the former section works perfectly for the Three-Body Problem in the plane because the averaged system is in fact completely integrable: after reduction by the symmetry of rotation, that is fixing the angular momentum and quotienting by the rotation group, one finds a system with only one degree of freedom, hence integrable. But a problem arises for the spatial problem: describing, at the end of section 132, the application to the Three-Body Problem of the formal integration of the secular system (what we have called "putting the system into Birkhoff normal form"), Poincaré is well aware that, in order to prove the existence of the formal change of variables he must know that, writing, as we have just done, the quadratic part of the averaged system as a sum of squares $\sum_{j=1}^{4} A_{j}\left(\tau_{j}^{2}+\sigma_{j}^{2}\right)$,

La seule hypothèse que nous devions faire, c'est qu'il n'y ait pas entre les quatre constantes $A_{1}, A_{2}, A_{3}, A_{4}$ de relation linéaire à coefficients entiers. La probabilité pour que cette relation existe est nulle, mais on peut encore se demander s'il n'y a pas une relation simple de cette forme qui soit assez près d'être satisfaite pour que les séries ne convergent plus que très lentement. On sait que Le Verrier a discuté cette question, mais il a dû la laisser indécise en ce qui concerne les planètes inférieures, parce que les masses en sont mal connues et que les coefficients $A$ dépendent de ces masses.

Il est clair que tout ce qui précède s'applique, sans qu'on ait rien à y changer, au cas où l'on aurait plus de trois corps 65

\footnotetext{
${ }^{65}$ The only hypothesis we had to make is that, between the four constants $A_{1}, A_{2}, A_{3}, A_{4}$, there be no linear relation with integer coefficients. The probability for such a relation to exist is equal to zero, but one can still ask whether there is no simple relation of this form which would be almost satisfied so that the series would converge very slowly. One knows that Le Verrier discussed this question but he had to leave it unsettled for the inner planets because the masses are not well known and the coefficients $A$ depend on these masses. It is clear that all of the above applies, without having to change anything, in case one would have more than three bodies.
} 
I must confess that I do not know of which series Poincaré says that they would "converge" very slowly, as a priori the averaged system is not integrable in the spatial case, but in any case, the phenomenon to which he assigned zero probability does indeed occur for the spatial problem as one has always two resonances between the secular frequencies $A_{i}$ :

1) the invariance under rotation forces one of the vertical frequencies, say $A_{4}$ to be equal to zero. This does not happen in the planar case because circles are invariant under a rotation of their plane. But in $\mathbb{R}^{3}$, the plane containing a pair of coplanar circles can still be rotated non trivially, which accounts for a symplectic eigenplane with 0 frequency.

2) whatever be the masses, one has

$$
A_{1}+A_{2}+A_{3}+A_{4}=0 .
$$

The first resonance is harmless. Indeed, it would disappear after reduction but there is more: the reduction is unnecessary because one can show that, precisely because of the symmetries of the problem, the terms in the Birkhoff normal form which would be affected by a denominator equal to zero are not present.

The second one is also harmless but in a subtler way. Discovered in full generality (i.e., for the spatial planetary $(1+n)$-body problem for any $n$ ) by Michael Herman, and known today under the name of Herman's resonance, it was implicitely known in the lunar theory since the beginning of the Newtonian theory through the fact that, if one takes into account only the terms of the first-order in the planetary masses, the theory of perturbation gives practically the same value of 18 years for the period of the direct motion of the lunar apogee and the retrograde motion of its line of nodes (see $\mathrm{AA}$ where it is pointed out that Poincaré mentions this resonance in [P7]).

Well aware of the first resonance (but somewhat forgetting it), Poincaré overlooked the second one, which makes his proof of the existence of Lindstedt series in the spatial case incomplete ${ }^{66}$ but, fortunately, the Herman resonance disappears after full reduction of the rotation symmetry, and this allows to restore the proof. More precisely (see [MRL]), in the Three-Body Problem, it spoils the Birkhoff normal form only from terms of order at least 10 in the eccentricities and inclinations and, even more, it does not appear at all in the full formal Birkhoff normal form after the partial reduction which consists in fixing only the direction of the angular momentum (we shall come back to this in section 11). Finally, it has no incidence on the dynamics and moreover, it disappears if one extends the averaging at the second order of the masses ${ }^{67}$ A striking illustration of this last fact is the difference between the value of 18 years for the period of the lunar apogee and the actual value of approximately 9 years 68 This discrepancy had casted a major doubt on the Newtonian theory until Clairaut explained in 1750 that taking into account some terms of higher order [ in the lunar problem, the small parameter $\mu$ is the ratio of mean motions of the Moon and the Sun] did restore the correct value of 9 years ${ }^{69}$

\footnotetext{
${ }^{66}$ It is this same mistake, overlooking the Herman resonance, that Arnold will make in 1963, see section $11 .$. the Oulipo would say that Poincaré had made a mistake by anticipation.

${ }^{67}$ In some sense, sticking to the frequencies at the secular singularity to build expansions is too rigid, in a way as rigid as were the old methods making Taylor expansions at fixed frequencies and hence introducing secular terms.

${ }^{68} \mathrm{On}$ the contrary, the value of 18 years for the period of the node is close to the actual one.

${ }^{69}$ For a full account of this important episode, see d'Al and the recension C6.
} 


\section{Periodic solutions 2) The source of complexity}

We have asserted in section 2.6 that the breaking of periodic invariant tori of the unperturbed system was the main cause of the extreme complexity of most of the solutions of the Three-Body Problem. This complexity is displayed on the one hand in the non-integrability and the divergence of Lindstedt series, both originating from the breaking of periodic invariant tori of the integrable approximations which gives rise to periodic solutions with non-zero exponents, on the other hand in the divergence of Bohlin's series which signs the splitting of separatrices. In the popular account [P6], also reproduced in [Ra1], Poincaré gives a very lucid account of the way he understands the role of periodic solutions:

Les équations différentielles du problème des trois corps admettent un certain nombre d'intégrales qui sont connues depuis longtemps; ce sont celles du mouvement du centre de gravité, celles des aires, celles des forces vives. Il était extrêmement probable qu'elles ne pouvaient avoir d'autres intégrales algébriques; ce n'est cependant que dans les dernières années que M. Bruns a pu le démontrer rigoureusement. Mais on peut aller plus loin; en dehors des intégrales connues, le problème des trois corps n'admet aucune intégrale analytique et uniforme; les propriétés des solutions périodiques et asymptotiques, étudiées avec attention, suffisent pour l'établir. On peut en conclure que les divers développements proposés jusqu'ici sont divergents ; car leur convergence entraînerait l'existence d'une intégrale uniforme 70

\subsection{Variational equations: exponents, stability, asymptotic solutions}

Initiating the study of the stability of periodic orbits, Poincaré introduces their characteristic exponents and their stable and unstable manifolds. The famous mistake in his 1889 prize memoir [P1], where he had thought he had proved stability in the Restricted Problem, is about the intersection of these manifolds (see [BG, Y $]$ ). We shall come back to this topic in section 9 .

Chapter IV begins with the definition of the variational equations. Given a particular solution $x(t)=\left(x_{1}(t), \ldots, x_{n}(t)\right)$ of a differential equation $\frac{d x}{d t}=X(x)$ defined in some domain of $\mathbb{R}^{n}$, the variational equation along $\varphi$ is obtained by writing that $x(t)+\xi$ is also a solution and ignoring the terms in $\xi=\left(\xi_{1}, \ldots, \xi_{n}\right)$ of order 2 or more:

$$
\frac{d \xi_{i}}{d t}=\frac{\partial X_{i}}{\partial x_{1}}(x(t)) \xi_{1}+\cdots+\frac{\partial X_{i}}{\partial x_{n}}(x(t)) \xi_{n} .
$$

In order to stress the importance of these equations, Poincaré recalls that Hill used them in his "admirable theory of the Moon": starting with his periodic solution (see 4.1) which already gave a reasonable value of the inequality called variation,

\footnotetext{
${ }^{70}$ The differential equations of the Three-Body Problem possess a number of integrals which have long been familiar; these are those of the motion of the center of mass, the area integrals, the energy. It was extremely unlikely that they could have other algebraic integrals; it is, however only in the recent years that Mr. Bruns has proved this rigorously. But we can go further; apart from the known integrals, the Three-Body Problem admits no analytic and uniform integral; a careful study of the properties of periodic and asymptotic solutions is enough to establish this. It can be concluded that the various developments proposed so far are divergent; for their convergence would imply the existence of a uniform integral.
} 
the study of the variational equations allowed him to compute the motions of the perigee and the node, and the coefficient of the evection.

Having defined the exponents $\alpha_{j}$ of a linear differential equations with periodic coefficients via Floquet's theory ${ }^{71}$, he turns his attention to the variational equation along a periodic solution of an autonomous differential equation: in this case, the exponents are the logarithms of the eigenvalues of the monodromy $d \varphi_{T}(x(t))$ at any point of the periodic solution; one of them is always equal to zero and moreover, to each independent first integral corresponds a zero eigenvalue and to each continuous symmetry corresponds a zero co-eigenvalue.

In the particular case of the (Hamiltonian) equations of dynamics

$$
\frac{d x_{i}}{d t}=-\frac{\partial F}{\partial y_{i}}, \quad \frac{d y_{i}}{d t}=\frac{\partial F}{\partial x_{i}},
$$

he notices that, writing $(\xi, \eta)$ and $\left(\xi^{\prime}, \eta^{\prime}\right)$ two solutions of the variational equations ${ }^{72}$

$$
\sum_{i=1}^{n} \frac{d}{d t}\left[\eta_{i}^{\prime} \xi_{i}-\xi_{i}^{\prime} \eta_{i}\right]=0,
$$

and analogous determinantal formulæ for $4,6, \ldots, 2 n$ solutions which are nothing but the preservation by the flow of the symplectic form and its powers ${ }^{73}$

Then, recalling that, if a linear transformation preserves a quadratic form, its characteristic polynomial is "reciprocal", he deduces 74 that the characteristic exponents are pairwise equal (in absolute value) and of opposite sign 75

The next step is to study the solutions which start close to the given periodic solution by writing power series for the solutions $\xi(t)$ of the (non linearized) equation $\frac{d}{d t}(x(t)+\xi)=X(x(t)+\xi)$. In chapter VII, the last chapter of the second volume of The New Methods, Poincaré shows the existence and analyticity with respect to a parameter of what we call today stable and unstable manifolds of a periodic solution, respectively associated with the set of exponents with negative or positive real part (the so-called hyperbolic part of the spectrum). He does so by writing down convergent series expansions for the families of solutions they contain. He does not

\footnotetext{
${ }^{71}$ Given a linear differential equation $\frac{d x}{d t}=A(t) x$ where the matrix $A(t)$ is periodic of period $T$ and a fundamental matrix solution $X(t)$, that is an invertible matrix whose columns are a basis of solutions of the equation, one notices that the columns of $X(t+T)$ are also solutions, hence that there exists a monodromy matrix $\mathcal{M}$ such that $X(t+T)=X(t) \mathcal{M}$. The set of exponents is the spectrum, deprived of the obvious eigenvalue 1 , of the monodromy matrix. It is well defined because a change of fundamental matrix solution leads to a conjugate monodromy matrix. Having recalled the theory, Poincaré cannot help adding:

Je n'insiste pas sur tous ces points de détail. Ces résultats sont bien connus par les travaux de MM. Floquet, Callandreau, Bruns, Stieltjes, et, si j'ai donné ici la démonstration in extenso pour le cas général, c'est que son extrême simplicité me permettait de le faire en quelques mots.

I do not insist on this minor point. These results are well known by the works of MM. Floquet, Callandreau, Bruns, Stieltjes and if if I have given here the proof in extenso for the general case, it is because its extreme simplicity allowed me to do it in a few words.

${ }^{72} \xi$ and $\eta$ being respectively the small increases of $x$ and $y$.

${ }^{73}$ For more on this, see section 8.1

${ }^{74}$ The proof is contested by A. Wintner in 1931 but it seems that Wintner forgot that the differential equation with periodic coefficients which is considered is the variational equation along a periodic solution of an autonomous equation.

${ }^{75}$ This theorem is rightly named by Kozlov "Poincaré-Lyapunov theorem". Indeed, in his book Lya, published in 1892 by the mathematical society of Kharkov, Lyapunov proves this theorem and he explains that the result was known to him before the publication of [P1], and that he had communicated it in February 1890 to the Kharkov mathematical society.
} 
discard the central part corresponding to exponents whose real part is zero but is aware that convergence could fail. We shall come back to these invariant manifolds in the particular case of the Restricted Problem in section 9.

Now comes the important fact heralded in section 2.6. for the unperturbed Hamiltonian $F_{0}(x)$ of the general problem of dynamics, the variational equation along any periodic solution is trivial and hence the exponents are all equal to zero. Showing that after adding the small perturbation $\mu F_{1}(x, y)$ periodic solutions with non trivial exponents will appear is at the root of the non-integrability of the problem. Indeed, to the families of periodic solutions of the equations of dynamics, which depend analytically on the small parameter $\mu$, are attached families of asymptotic solutions but, as all the exponents vanish when $\mu=0$, the exponents and the solutions of the variational equations have series expansions not in powers of $\mu$ but in powers of its square root $\sqrt{\mu}$. Poincaré then deduces (after some work!) that the asymptotic solutions may be expressed as series expansions in powers $\sqrt{\mu}$, with coefficients given by series in powers of variables $w_{j}=A_{j} e^{\alpha_{i} t}$ (where the $\alpha_{j}$ are the characteristic exponents of the periodic solution), $e^{i t}$ and $e^{-i t}$; he shows that these series diverg $e^{76}$ but that, while divergent, they are asymptotic series in the sense he will formalize in chapter VIII, which opens the second volume:

On peut donc dire que les séries que nous avons obtenues dans le $\mathrm{n}^{o} 108$ représentent les solutions asymptotiques pour les petites valeurs de $\mu$ de la même manière que la série de Stirling représente les fonctions eulériennes. 77

The intuitive idea behind these expansions is explained on the following figure in the case of 2 degrees of freedom: a continuous family of periodic solutions of the unperturbed problem gives rise to isolated elliptic and hyperbolic periodic solutions and hence, in the first approximation, to penduli of width $\sqrt{\mu}$ (opening of a resonance).

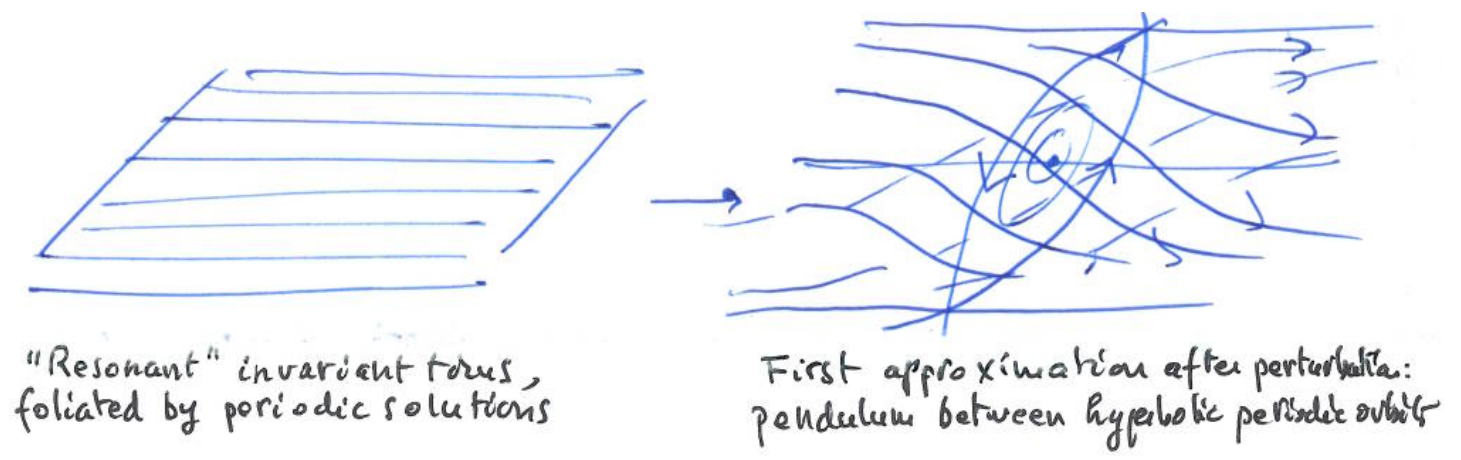

Figure 7: Opening of a resonance.

This is nothing but enriching Figure 3 with invariant manifolds: analytically, it corresponds to the family of integrable Hamiltonians

$$
F_{0}(x)+\mu F_{1}(x, y)=\frac{1}{2}\left(x_{1}^{2}+\cdots+x_{n}^{2}\right)-\mu \omega^{2} \cos y_{1}
$$

We shall come back to this situation when studying Bohlin series in section 7

\footnotetext{
${ }^{76}$ Another proof, based on the divergence of the Bohlin series, will be given at the very end of volume II.

${ }^{77}$ Hence one may say that that the series that we have obtained in $\mathrm{n}^{\circ} 108$ represent the asymptotic solutions for the small values of $\mu$ in the same way as the Stirling series represent the Eulerian functions.
} 


\subsection{Non-existence of uniform first integrals}

Bruns had shown ${ }^{78}$ the non-existence of first integrals of the Newtonian Three-Body Problem algebraic in the velocities, which do not arise from the ones which are consequence of the symmetries of the problem: energy and angular momentum. Using a completely different method, intimately related to the behaviour of periodic solutions, Poincaré shows in chapters V and VI the non-existence of new first integrals analytic in $x, y$ and $\mu$, that is which are analytic in the (sufficiently small) masses of the planets. Comparing his result with Brun's theorem, he writes at the end of section 85:

Le théorème qui précède est plus général en un sens que celui de M. Bruns, ... Mais, en un autre sens, le théorème de M. Bruns est plus général que le mien; j'établis seulement, en effet, qu'il ne peut pas exister d'intégrale algébrique pour toutes les valeurs suffisamment petites des masses; et M. Bruns démontre qu'il n'en existe pour aucun système de valeurs des masses 79

If "generic" periodic solutions (i.e., solutions with non trivial exponents) were dense in the phase space, or even only if they formed a uniqueness set ${ }^{80}$ this would imply analytic non-integrability. This is because the existence of a complete set of almost everywhere independent commuting integrals ${ }^{81}$ implies that every periodic orbit on which the integrals are independent has all its exponents equal to zero. Unfortunately, even if it is likely to be true, this property is not proved, hence the necessity of more analytic methods of proving non-integrability.

The actual proo ${ }^{82}$ rests on a delicate analysis of the abundance of non-zero coefficients in the Fourier expansion in the angles $y$ for the perturbing function $\mu F_{1}+\cdots$,

\footnotetext{
${ }^{78}$ Actually, Brun's paper contained a mistake which was noticed and corrected by Poincaré in [P9]. The conclusion of this paper is one more characteristic example of Poincaré's style:

Le résultat de M. Bruns se trouve confirmé; je suis heureux d'avoir pu compléter son élégante analyse sur un point de détail.

Hence the result of Mr. Bruns is confirmed. I am pleased to have been able to complete his elegant analysis on a small technical point.
}

It has nevertheless some savour to compare this modest ending with the corresponding statement in Poincaré's analysis of his own works:

On sait que Bruns a démontré que le problème des trois corps ne saurait admettre d'autre intégrale algébrique que les intégrales classiques. Malheureusement dans sa démonstration subsistait une lacune grave et particulièrement délicate à combler. J'ai été assez heureux pour mettre la belle et ingénieuse démonstration de M. Bruns à l'abri de toute objection.

One knows that Bruns has proved that the Three-Body Problem could not admit other algebaic integral than the classical ones. Unfortunately a serious gap remained in his proof which was very difficult to fill. I was fortunate enough to put the beautiful and clever proof of Mr. Bruns away from any objection.

For a complete proof of Brun's theorem, see [JT]; for a generalization (not really checked), see $[\mathrm{Pa}$.

${ }^{79}$ The theorem above is in some sense more general than the one of M. Bruns, ... But, in another sense, the theorem of M. Bruns is more general than mine; indeed I prove only that there does not exist algebraic integrals valid for all sufficiently small values of the masses; and M. Bruns proves that such integral exists for no system of values of the masses whatsoever.

80 i.e., a set such that an analytic function which is equal to zero on the set is identically zero.

${ }^{81}$ that is integrals $G, H, K, \ldots$, whose Hamiltonian flows all commute and hence define a possibly singular foliation of the phase space by invariant Lagrangian tori; this is equivalent (see [A2]) to the vanishing of all their Poisson brackets $\{G, H\}=\sum \frac{\partial G}{\partial x_{j}} \frac{\partial H}{\partial y_{j}}-\sum \frac{\partial H}{\partial x_{j}} \frac{\partial G}{\partial y_{j}},\{\mathrm{G}, \mathrm{K}\},\{\mathrm{H}, \mathrm{K}\}, \ldots$

${ }^{82}$ Comparing, in section 85, this proof to the one he had given In the Memoir (see 6.5), Poincaré writes: Dans mon Mémoire des Acta Mathematica (t. XIII), je me suis servi pour établir le même point de l'existence des solutions périodiques et du fait que les exposants caractéristiques ne sont pas nuls. La démonstration que je donne ici ne diffère de celle des Acta que par la forme, mais elle se prête mieux à la généralisation qui va suivre.

In my Memoir of the Acta Mathematica (t. XIII), in order to establish the same point, I used the 
which amounts essentially to proving the "generic" behaviour of periodic solutions, that is the fact that their exponents are non-zero, and forbids them to form continua filling "periodic" invariant tori as is the case when $\mu=0$ (see again 2.6).

In order to show how resonances do appear in the reasoning, let us examine the non-degenerate case, where we suppose that the Hessian of the unperturbed Hamiltonian $F_{0}(x)$ does not vanish, that is when the frequency map $x \mapsto \frac{\partial F_{0}}{\partial x}(x)$ is a diffeomorphism. Supposing that there is an integral $\Phi=\Phi_{0}+\mu \Phi_{1}+\cdots$, Poincaré develops the identity of commutation 83

$$
\{F, \Phi\}=\left\{F_{0}, \Phi_{0}\right\}+\mu\left(\left\{F_{0}, \Phi_{1}\right\}+\left\{F_{1}, \Phi_{0}\right\}\right)+\cdots=0
$$

The first relation implies that $\Phi_{0}$ does not depend on the angles $y$. If the Fourier expansions of $F_{1}$ and $\Phi_{1}$ are respectively

$$
F_{1}(x, y)=\sum_{m \in \mathbb{Z}^{n}} B_{m}(x) e^{i m \cdot y}, \quad \Phi_{1}(x, y)=\sum_{m \in \mathbb{Z}^{n}} C_{m}(x) e^{i m \cdot y}
$$

the second relation means that the Fourier coefficients of $\Phi_{1}$ must satisfy

$$
\forall m, \quad C_{m} \sum m_{j} \frac{\partial F_{0}}{\partial x_{j}}-B_{m} \sum m_{j} \frac{\partial \Phi_{0}}{\partial x_{j}}=0
$$

that is: $\sum m_{j} \frac{\partial \Phi_{0}}{\partial x_{j}}(x)=0$ each time an action belongs to the secular se ${ }^{84}$

$$
\mathcal{S}=\left\{x, \exists m=\left(m_{1}, \ldots, m_{n}\right) ; \sum m_{j} \frac{\partial F_{0}}{\partial x_{j}}(x)=0 \quad \text { and } \quad B_{m}(x) \neq 0\right\}
$$

Notice that, the equation above being homogeneous in $m$, the relevant feature of the perturbing function is not whether an individual Fourier coefficients $B_{m}$ is different from 0 but whether a class contains a non zero coefficient, where $B_{m^{\prime}}$ and $B_{m^{\prime \prime}}$ are in the same class if $m_{1}^{\prime} / m_{1}^{\prime \prime}=m_{2}^{\prime} / m_{2}^{\prime \prime}=\cdots=m_{n}^{\prime} / m_{n}^{\prime \prime}$.

Figure 8 illustrates the definition of a class in the case of two degrees of freedom: the level curves of $F_{0}$ and $\Phi_{0}$ must be tangent at each point of the secular set. If this set is big enough, for example if it is dense or if it is a uniqueness set, this will imply that the level sets of $F_{0}$ and $\Phi_{0}$ coincide, and hence that $\Phi_{0}$ is a function of $F_{0}$. In this case, after substracting $\Phi_{0}$ and dividing by $\mu$ one has a new integral of the form $\Phi_{1}+\mu \Phi_{2}+\ldots$ and the rest of the proof is similar.

existence of periodic solutions and the fact that the characteristic exponents are non zero. The proof I give here differs from the one in the Acta only by the form, but it is more apt to the following generalization.

\footnotetext{
${ }^{83}$ Recall that $\{F, G\}=\sum \frac{\partial F}{\partial x_{j}} \frac{\partial G}{\partial y_{j}}-\sum \frac{\partial G}{\partial x_{j}} \frac{\partial F}{\partial y_{j}}$ is the Poisson bracket.

${ }^{84}$ Poincaré says that a Fourier coefficient $B_{m}$ of the perturbing function becomes secular if the actions $x$ are such that $\sum m_{j} \frac{\partial F_{0}}{\partial x_{j}}(x)=0$.
} 


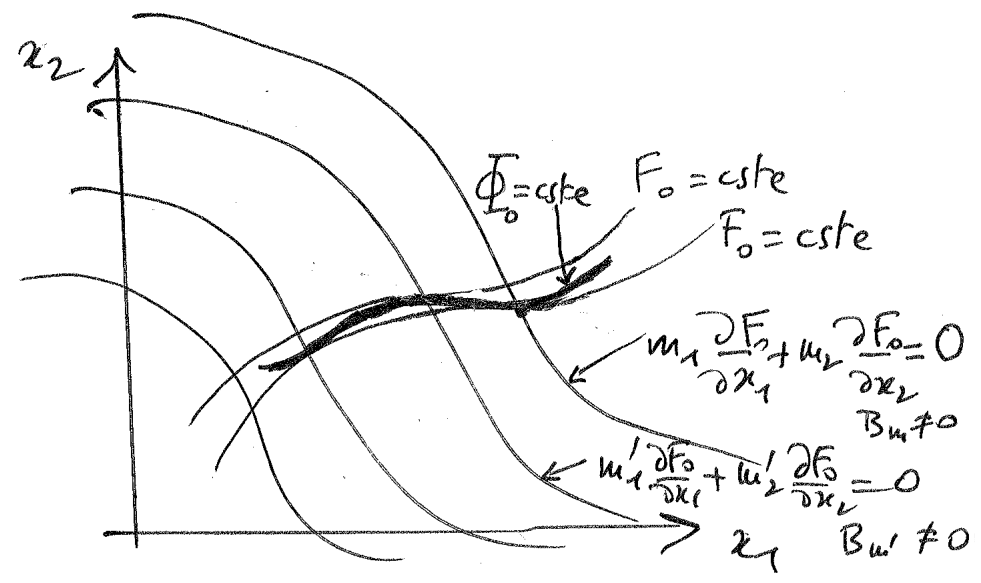

Figure 8: Being tangent along the secular set.

When there are more than two degrees of freedom, the proportionnality of the gradients of $F_{0}$ and $\Phi_{0}$ at $x$ will now take place on the Poincaré set

$\mathcal{P}=\left\{x, \exists m^{(1)}, \ldots, m^{(n-1)} \in \mathbb{Z}^{n}\right.$ independent; $\left.\forall k, \sum_{j} m_{j}^{(k)} \frac{\partial F_{0}}{\partial x_{j}}(x)=0, B_{m^{(k)}}(x) \neq 0\right\}$.

These values $x$ of the actions define a completely resonant (i.e., filled with periodic solutions) torus of the unperturbed system and the fact that the $B_{m^{(k)}}$ are non zero, means that such a torus will be broken by the perturbation. Good references for this, with many examples, are [Koz, $\mathrm{AKN}]$.

As usual, this simple theory applies to the case of the Restricted Three-Body Problem, the one studied in the Memoir ${ }^{85}$ but not to the general Three-Body Problem because of the degeneracy of the Kepler Problem. The relevant Fourier expansions of $F_{1}$ and $\Phi_{1}$ being $\sum B_{m_{1}, m_{2}} e^{i\left(m_{1} l_{1}+m_{2} l_{2}\right)}$ and $\sum C_{m_{1}, m_{2}} e^{i\left(m_{1} l_{1}+m_{2} l_{2}\right)}$, where $l_{1}, l_{2}$ are the two mean anomalies (the fast angles) and $B_{m}, C_{m}$ depend on the fast actions and all the slow variables, the slow variables introduce new terms in the relations between $B_{m}$ and $C_{m}$ and the Poincaré set must be defined in a subtler way. This is done in chapter V, while the long chapter VI , "Développement de la fonction perturbatrice", is devoted to showing that the Fourier coefficients of the expansion in the mean anomalies of the perturbing function ${ }^{86}$ satisfy the conditions established in chapter V: associating to a Fourier expansion the function of complex variables obtained by replacing complex exponentials by complex variables, Poincaré generalises to functions of two complex variables a method by Darboux, first used by Flamme, which relates the behaviour of the high rank Fourier coefficients of a periodic function $\sum_{n \in \mathbb{Z}} B_{n} e^{i n \varphi}$ to the singularities of the complex function $\sum_{n \in \mathbb{Z}} B_{n} z^{n}$. After 66 pages of virtuose use of deformations of integration contours where he stresses the role of the coalescence of singular points of the integrand, he may conclude:

La non-existence des intégrales uniformes se trouve ainsi démontrée. ${ }^{87}$

\footnotetext{
${ }^{85}$ See section 9 the Hamiltonian $F_{0}(L, G)=-\frac{K}{L^{2}}+G$ is degenerate (its Hessian vanishes) but Poincaré's trick of replacing it by $\exp F_{0}$ eliminates the degeneracy without modifying the integrability properties. Moeover, it follows from the form, computed by Leverrier (see Cha ) of he Fourier expansion of the perturbing function, that the secular set is dense, hence the proof of the non-integrability.

${ }^{86}$ In particular the principal part, that is the inverse distance beween the two planets (see section 2.3 .

${ }^{87}$ The non-existence of uniform integrals is thus rigorously proved.
} 


\subsection{Divergence of Lindstedt series}

It is in chapter XIII that Poincaré proves the divergence of the series with varying frequencies. It is essentially a consequence of the proof of the non-integrability result which asserts the breaking of the periodic invariant tori of the unperturbed system: if the Lindstedt series with varying frequencies did converge, this would imply the existence of such periodic tori, hence a contradiction.

Even if he considers it very unlikely, he is aware of the possibility of the convergence of Lindstedt series with fixed frequencies "satisfying some arithmetic conditions"; this is the famous paragraph 149, which I quote in section 11, putting it in the light of Arnold's "theorem" on the stability of planetary systems.

Remark. In his proofs of non-integrability and hence of the divergence of Lindstedt series, Poincaré does not make use of the subtler phenomena of separatrix splitting (see the question at the end of section 10.1). A proof of analytic non-integrability based on the existence of such transversal homoclinic intersections was done by Jürgen Moser in an example due to Sitnikov, with two degrees of freedom [M5].

\subsection{Back to the old methods: the "Lessons of Celestial Mechanics"}

As already mentioned in section 5, Poincaré was well aware of the different aims of geometers and astronomers; he knew perfectly that the divergence of the Lindstedt series did not diminish their practical interest and that the old methods were not to be rejected readily. In 1898, he had published in the "Bulletin Astronomique" P11 a long article explaining how to recover the classical series expansions of the astronomers from the "new ones", insisting in particular on the fact that, according to the purpose being sought (a very precise representation of the position of the celestial bodies during a short time or a less precise representation but during a very long time) the dominant terms in the series are not the same. A testimony of this attitude is the courses given in the Sorbonne and published under the name of Lessons of celestial mechanics [P12 between 1905 and 1910. Much less known 88 than the New Methods, this other great book on Celestial Mechanics is the redaction by people in the audience of courses at the Sorbonne; directly aimed at students, it examines the classical methods of perturbation. In the introduction, Poincaré warns his readers:

Je n'emprunte aux méthodes nouvelles que leurs résultats essentiels, ceux qui sont susceptibles d'une application immédiate, en m'efforçant de les rattacher le plus intimement possible à la méthode classique de la variation des constantes.

D'un autre côté, Tisserand s'est constamment préoccupé de reproduire aussi fidèlement qu'il a pu la pensée des fondateurs de la Mécanique céleste et, en effet, son Livre nous la rend tout entière sous une forme condensée. Je n'avais pas à refaire ce qu'il avait fait et bien fait 89

As The New Methods, the Lessons have three volumes: the first one, General theory of planetary perturbations (1905), starts with a thorough study of the general

\footnotetext{
${ }^{88}$ in particular to the author, which explains the brevity of this section.

${ }^{89}$ To the new methods, I borrow only the essential results, those capable of immediate application, while striving to relate them as closely as possible to the classical method of the variation of constants. On the other hand, Tisserand paid constant attention to reproducing as faithfully as possible the thought of the founders of Celestial mechanics and, indeed, his book restitutes it in a compact form. I had no need to do again what he had done and done well.
} 
properties of the Three-Body Problem and, in particular of the various sytems of coordinates convenient to study the planetary problem; this is followed by a remarkable exposition of the Keplerian elliptic motion, with in particular a complete proof of the analyticity 90 of what we have called the Poincaré coordinates (see 2.2). The chapters are: Lagrange's method; The Restricted Problem; Theory of secular perturbations; Poisson theorem; Symmetries in the developments, periodic solutions; Delaunay's method. The second volume is divided into two parts: 1) Développement of the perturbing function (1907), 2) Theory of the Moon (1909), while the third one is devoted to the Theory of tides (1910).

\subsection{Complexifying Poincaré's method}

The main new tool in order to prove non-integrability results was introduced by Ziglin in [Z]: starting with some particular solution of the equations, the obstruction to the existence of first integrals meromorphic in a neighborhood of this solution is found in the monodromy group of the variational equation along the complexification of the given solution. As Alain Albouy likes to say, Ziglin's method can be viewed as a complexification of the method originally used by Poincaré in his Memoir to prove non-integrability: any first integral was shown to be dependent of the Hamiltonian along any "generic" (i.e., with non trivial exponents) periodic solution. The proof is extremely simple: Let $F(x, y)$ be a Hamiltonian, $\varphi_{t}$ the flow of the corresponding Hamiltonian vector field ${ }^{91} X_{F}$ and $\Phi$ a first integral of these equations ${ }^{92}$ If the solution $\varphi_{t}\left(z_{0}\right)$ is $T$-periodic, i.e., if $\varphi_{T}\left(z_{0}\right)=z_{0}$, one deduces from the constancy of $F$ and $\Phi$ and the chain rule on the one hand, differentiating with respect to $t$ the identity $\varphi_{T} \circ \varphi_{t}\left(z_{0}\right)=\varphi_{t}\left(z_{0}\right)$ on the other hand, that the monodromy matrix $d \varphi_{T}\left(z_{0}\right)$ of the periodic solution $\varphi_{T}\left(z_{0}\right)$ has 2 co-eigenvectors $d F\left(z_{0}\right)$ and $d \Phi\left(z_{0}\right)$ with co-eigenvalue 1 and one eigenvector $X_{F}\left(z_{0}\right)$ with eigenvalue 1:

$$
d F\left(z_{0}\right) d \varphi_{T}\left(z_{0}\right)=d F\left(z_{0}\right), d \Phi\left(z_{0}\right) d \varphi_{T}\left(z_{0}\right)=d \Phi\left(z_{0}\right), d \varphi_{T}\left(z_{0}\right) X_{F}\left(z_{0}\right)=X_{F}\left(z_{0}\right) .
$$

Moreover $d F\left(z_{0}\right) X_{F}\left(z_{0}\right)=d \Phi\left(z_{0}\right) X_{F}\left(z_{0}\right)=0$, from which one deduces that either this periodic solution has at least three exponents equal to zero, and hence is not generic, or the derivatives $d F\left(z_{0}\right)$ and $d \Phi\left(z_{0}\right)$ are dependent. After complexification, the variational equation becomes a linear differential equation along a Riemann surface and Ziglin's criterion of non-integrability involves the non commutation of some elements of the monodromy group of this equation which leads to the branching of solutions. A great advantage of the complexification is that the periodic solution is no more required to be generic. A differential Galois group version of this theorem was developed by Morales and Ramis [MR], and extended to variational equations of higher order by Morales-Ramis-Simó [MRS]. The criterion is the non-abelianity of the Lie algebra of the differential Galois group. Specific applications of these methods to the three (or more)-body problem were given by D. Boucher, J.J. Morales, A. Tsygvintsev, C. Simó, S. Simon, T. Combot, J.A. Weil, ... References will be found in $\mathrm{Au}$ ] and the more recent [Com1. See also Com2 which helps justifying the use of the Morales-Ramis theorem in most of the previous papers.

\footnotetext{
${ }^{90}$ An amusing story: it happenned to me to be the referee of a paper showing the analyticity of Poincaré's coordinates for the Kepler Problem. The result was less strong than Poincaré's and I had simply to refer to the first volume of the Lessons.

${ }^{91}$ If $z=(x, y), X_{F}(z)=\left(-\frac{\partial F}{\partial y}(x, y), \frac{\partial F}{\partial x}(x, y)\right)$ and $\frac{d \varphi_{t}}{d t}(z)=X\left(\varphi_{t}(z)\right)$. Recall that the monodromy is $d \varphi_{T}\left(z_{0}\right)$.

${ }^{92}$ The situation is of course symmetric and amounts to the vanishing of the Poisson bracket $\{F, \Phi\}$.
} 


\section{Resonances 1) Bohlin series}

Resonances play a major role in celestial mechanics; the earliest mathematical studies seem to be those of Laplace on the 4:2:1 resonance between the Jovian satellites Ganymede, Europa and Io, and the 5:2 resonance between Jupiter and Saturn.

The neighborhood of a single resonance is, in the first approximation, well described by the equations of the product of simple rotators by a simple pendulum ${ }^{93}$ As written by Tisserand [Ti]:

If a dynamical system is in a state of resonance, then there exists a linear combination of the angle-variables, the so-called critical argument, that behaves like the angular coordinate of the simple pendulum. Depending on the initial conditions, it either librates about the point of stable equilibrium or it circulates around the clock.

The fundamental fact is that this approximate system is completely integrable, which is no more true in case of multiple resonance (see [GSV]).

\subsection{Circulation, libration, separatrix: what is a Bohlin series?}

Reprenons les hypothèses et les notations du $n^{o} 125$. Nous avons vu que dans l'application de la méthode du $\mathrm{n}^{o} 125$ il s'introduisait des diviseurs de la forme

$$
n_{1}^{0} m_{1}+n_{2}^{0} m_{2}+\cdots+n_{n}^{0} m_{n}
$$

les $m_{i}$ étant entiers ${ }^{94}$

Il en résulte que cette méthode devient illusoire quand l'un de ces diviseurs devient très petit 95

This quotation opens the long chapter XIX, devoted to simple resonances. The simplest illustration of the phenomena which are studied is the birth of a simple pendulum from a linear rotator:

$$
F\left(x_{1}, \ldots, x_{n}, y_{1}, \ldots, y_{n}\right)=\frac{1}{2}\left(x_{1}^{2}+x_{2}^{2}+\cdots+x_{n}^{2}\right)+\mu \cos y_{1} \quad(\mu \geq 0) .
$$

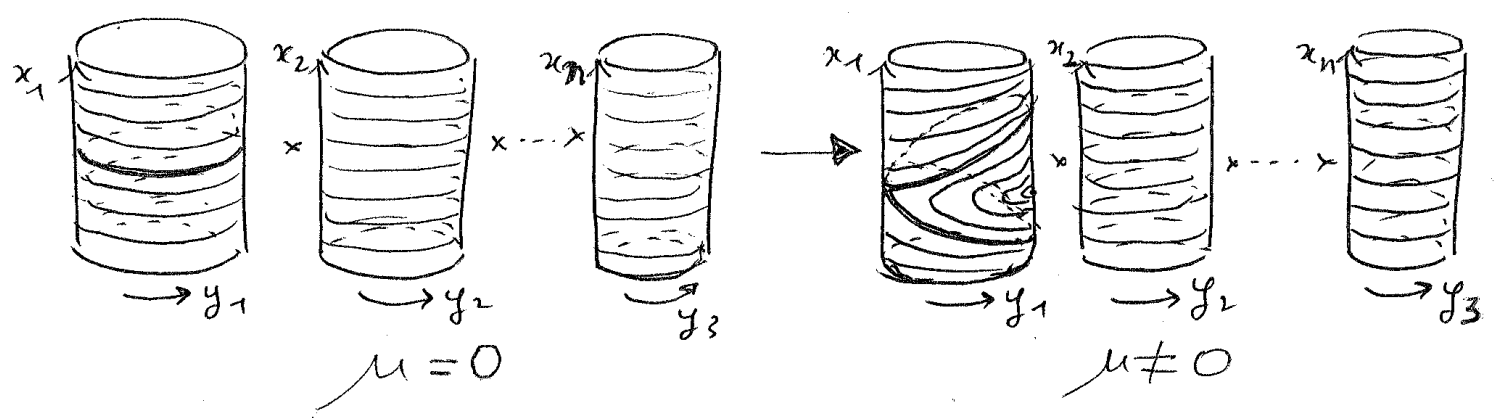

Figure 9: Opening of a simple resonance.

\footnotetext{
${ }^{93}$ Too many physics students ignore the phase portrait of the simple pendulum!

${ }^{94}$ I remind you that with the notations of the general problem of dynamics (see section 2.5) and my sign conventions, $n_{i}^{0}=\frac{\partial F_{0}}{\partial x}\left(x^{0}\right)$ is the frequency of the invariant torus of the perturbed system with action $x=x^{0}$.

${ }^{95}$ Let us come back to the hypotheses and notations of $\mathrm{n}^{o} 125$. We have seen that, in the application of the method of $\mathrm{n}^{o} 125$, small denominators came up, of the form $n_{1}^{0} m_{1}+n_{2}^{0} m_{2}+\cdots+n_{n}^{0} m_{n}$, where the $m_{i}$ are integers. It results that this method become illusive when one of these divisors becomes very small.
} 
This is the case where $x^{0}=\left(0, x_{2}^{0}, \ldots, x_{n}^{0}\right)$ and $\left(m_{1}, m_{2}, \ldots, m_{n}\right)=(1,0, \ldots, 0)$. In other words, the resonant angle is ${ }^{96} \sum m_{i} y_{i}=y_{1}$. Note that the actions $x_{2}, \ldots, x_{n}$ are integrals of the motion. We shall fix $x^{0}=\left(0, x_{2}^{0}, \ldots, x_{n}^{0}\right)$ and write

$$
c_{0}=F_{0}\left(x^{0}\right)=\frac{1}{2}\left(\left(x_{2}^{0}\right)^{2}+\cdots+\left(x_{n}^{0}\right)^{2}\right) .
$$

In this integrable model, three types of solutions coexist, depending on the value $C$ of the energy:

the circulation type, if $C-c_{0}>\mu$;

the libration type, if $-\mu<C-c_{0}<\mu$,

the separatrix type if $C-c_{0}=\mu$.

In the circulation range, the corresponding invariant tori are Lagrangian graphs and hence solutions of the Hamilton-Jacobi equation (compare with section 5.4)

$$
F\left(\frac{\partial S}{\partial y_{1}}(C, y), \frac{\partial S}{\partial y_{2}}(C, y), \ldots, \frac{\partial S}{\partial y_{n}}(C, y), y_{1}, y_{2}, \ldots, y_{n}, \mu\right)=C
$$

The solutions

$$
S(C, y)=x_{2}^{0} y_{2}+\cdots+x_{n}^{0} y_{n} \pm \int \sqrt{2\left(C-c_{0}-\mu \cos y_{1}\right)} d y_{1}
$$

admit an expansion in series of powers of $\mu$ (i.e., Lindstedt series) of the form $S=$ $\pm \sqrt{2 C} \sum_{k} \varphi_{k}\left(y_{1}\right)(\mu / C)^{k}$ but, as Poincaré notices, this expansion becomes useless in practice if $C-c_{0}$ is too close to $\mu$ and the expansion in powers of $\sqrt{\mu}$ has to be preferred.

In the libration range, the same holds true provided we first change variables in a way which turns the corresponding invariant tori into graphs.

For the separatrix type, which corresponds to any action $x^{0}=\left(0, x_{2}^{0}, \ldots, x_{n}^{0}\right)$ at which $n_{1}^{0}=\frac{\partial F_{0}}{\partial y_{1}}\left(x^{0}\right)$ vanishes, the situation is different. Indeed, the solution of the Hamilton-Jacobi equation satisfies

$$
\begin{aligned}
S\left(c_{0}+\mu, y\right) & =x_{2}^{0} y_{2}+\cdots+x_{n}^{0} y_{n} \pm \int \sqrt{2 \mu\left(1-\cos y_{1}\right)} d y_{1} \\
& =x_{2}^{0} y_{2}+\cdots+x_{n}^{0} y_{n} \pm 2 \sqrt{\mu} \int \sin \frac{y_{1}}{2} d y_{1} \\
& =x_{2}^{0} y_{2}+\cdots+x_{n}^{0} y_{n} \pm 4 \sqrt{\mu} \cos \frac{y_{1}}{2} .
\end{aligned}
$$

Notice that $S$ is no more $2 \pi$-periodic but only $4 \pi$-periodic in $y_{1}$. It is the image of an immersed torus which covers twice the circulation tori (see Figure 9). The two solutions are deduced from each other by a translation of $2 \pi$.

\section{2 "Bohlin method" in the non-degenerate case}

What Poincaré calls the Bohlin method, a conceptual improvement of the method used by Delaunay in his study of the motion of the Moon, was discovered in 1888 by

\footnotetext{
${ }^{96}$ This choice of the resonant angle is not a restriction as one can reduce the general case to this one by a linear symplectic change of coordinates.
} 
K. Bohlin Boh and Poincaré independently (see the introduction of the memoir; in The New Methods, Poincaré acknowledges a slight anteriority of Bohlin). Supposing that $F_{0}$ depends on all the action variables 97 and that

$$
n_{1}^{0}=\frac{\partial F_{0}}{\partial x_{1}}\left(x^{0}\right)=0
$$

is the sole linear relation between the frequencies ${ }^{98}$ it consists, in each of the three cases, in searching for solutions of the above Hamilton-Jacobi equation ${ }^{99}$ which are formal series of the form

$$
S=S_{0}+S_{1} \sqrt{\mu}+S_{2} \mu+S_{3} \mu \sqrt{\mu}+\cdots, C=C_{0}+C_{2} \mu+C_{4} \mu^{2}+\cdots
$$

with

$$
\forall i, \frac{\partial S_{0}}{\partial y_{i}}=x_{i}^{0} \quad \text { and } \quad \frac{\partial S_{p}}{\partial y_{i}} \text { periodic. }
$$

Refering to [S3] for an analysis of some of the obscurities in Poincaré's exposition which I shall not discuss in depth, I shall be content with showing how the three types of solutions appear in the equations, insisting more on the separatrix case, which plays a prominent role in sections 10.1 and 10.2 .

An immediate identification leads to the following equations:

$$
\left\{\begin{array}{l}
F_{0}\left(x_{1}^{0}, x_{2}^{0}, \ldots, x_{n}^{0}\right)=C_{0} \\
\sum n_{i}^{0} \frac{\partial S_{1}}{\partial y_{i}}=0 \\
\sum n_{i}^{0} \frac{\partial S_{2}}{\partial y_{i}}+\frac{1}{2} \sum \frac{\partial^{2} F_{0}}{\partial x_{i} \partial x_{k}} \frac{\partial S_{1}}{\partial y_{i}} \frac{\partial S_{1}}{\partial y_{k}}+F_{1}\left(x^{0}, y\right)=C_{2} \\
\sum n_{i}^{0} \frac{\partial S_{3}}{\partial y_{i}}+\frac{1}{2} \sum \frac{\partial^{2} F_{0}}{\partial x_{i} \partial x_{k}} \frac{\partial S_{1}}{\partial y_{i}} \frac{\partial S_{2}}{\partial y_{k}}+\Phi_{3}=0 \\
\sum n_{i}^{0} \frac{\partial S_{4}}{\partial y_{i}}+\frac{1}{2} \sum \frac{\partial^{2} F_{0}}{\partial x_{i} \partial x_{k}} \frac{\partial S_{1}}{\partial y_{i}} \frac{\partial S_{3}}{\partial y_{k}}+\Phi_{4}=C_{4} \\
\ldots
\end{array}\right.
$$

where, following Poincaré, I have denoted by $\Phi_{3}, \Phi_{4}$ known functions and adopted conventions for the summations which the reader will guess easily.

From the hypothesis that $n_{1}^{0}=0$ is the sole linear relation with integer coefficients between the frequencies, we deduce from the second equation that $S_{1}$ is necessarily of the following form:

$$
S_{1}=\alpha_{1} y_{1}+\alpha_{2} y_{2}+\cdots+\alpha_{n} y_{n}+f\left(y_{1}\right)
$$

where the derivative $f^{\prime}$ of $f$ is periodic.

Given a function $U(y)$, all of whose derivatives $\frac{\partial U}{\partial y_{i}}$ are periodic,

$$
U=\sum \alpha_{i} y_{i}+\sum_{p=\left(p_{1}, \ldots, p_{n}\right) \in \mathbb{Q}^{n}} a_{p} \sin \left(\sum p_{i} y_{i}\right)+\sum_{p=\left(p_{1}, \ldots, p_{n}\right) \in \mathbb{Q}^{n}} b_{p} \cos \left(\sum p_{i} y_{i}\right),
$$

\footnotetext{
${ }^{97}$ This will be pertinent in the case of the Restricted Problem.

${ }^{98} \mathrm{By}$ a symplectic change of coordinates one reduces any resonance relation $\sum m_{i} n_{i}^{0}=0, m_{i} \in \mathbb{Z}$, to $n_{1}=0$.

${ }^{99}$ After changing coordinates in the case of libration.
} 
as Poincaré we let

$$
[U]=\sum \alpha_{i} y_{i}+\sum_{p_{1} \in \mathbb{Q}} a_{\left(p_{1}, 0, \ldots, 0\right)} \sin \left(p_{1} y_{1}\right)+\sum_{p_{1} \in \mathbb{Q}} b_{\left(p_{1}, 0 \ldots, 0\right)} \cos \left(p_{1} y_{1}\right),
$$

the sum of the secular and resonant terms in $U$. It follows from this definition that, for any such $U$,

$$
\sum n_{i}^{0} \frac{\partial[U]}{\partial y_{i}}=\sum n_{i}^{0} \alpha_{i}
$$

is a constant. Hence one deduces from the third equation, that (I follow Poincaré's notation for the constants):

$$
\frac{1}{2}\left[\sum \frac{\partial^{2} F_{0}}{\partial x_{i} \partial x_{k}} \frac{\partial S_{1}}{\partial y_{i}} \frac{\partial S_{1}}{\partial y_{k}}\right]=C_{2}^{\prime}-\left[F_{1}\right]\left(x^{0}, y\right),
$$

which, given the expression of $S_{1}$, is an equation of the form

$$
A f^{\prime 2}+2 B f^{\prime}+D=C_{2}^{\prime}-\left[F_{1}\right]\left(x^{0}, y\right)
$$

the coefficients $A, B, D$ depending on the $\alpha_{i}$ which we can choose as we please. Choosing all the $\alpha_{i}$ equal to 0 , this implies $C_{2}^{\prime}=C_{2}$ and the formula defining $f^{\prime}$ becomes:

$$
A f^{\prime 2}=C_{2}-\left[F_{1}\right]\left(x^{0}, y\right) .
$$

The three cases are respectively

circulation: $C_{2}>\max \left[F_{1}\right], \quad$ libration: $C_{2}<\max \left[F_{1}\right], \quad$ separatrix: $C_{2}=\max \left[F_{1}\right]$.

In the separatrix case, the formula $f^{\prime}=\sqrt{A^{-1}\left(C_{2}-\left[F_{1}\right]\right)}$ defines two $4 \pi$-periodic functions of $y_{1}$.

This is only the beginning of the story which leads to proving the existence of the $S_{p}$, whose partial derivatives are $4 \pi$-periodic in $y_{1}$ and $2 \pi$-periodic in $y_{2}, \ldots, y_{n}$. Indeed, some work is needed to show that the constants $C_{4}, \ldots$ can be chosen in such a way that the $\frac{\partial\left[S_{p}\right]}{\partial y_{1}}$ remain finite; this is not obvious because in the equation $\frac{\partial^{2} F_{0}}{\partial x_{1}^{2}}\left(x^{0}\right) \frac{\partial S_{1}}{\partial y_{1}} \frac{\left[S_{p}\right]}{\partial y_{1}}=\cdots$, the term $\frac{\partial S_{1}}{\partial y_{1}}$ vanishes twice per period. Here is the way, at the end of section 209, Poincaré describes the formal penduli defined by the solutions of separatrix type that he has just found for the Hamilton-Jacobi equation:

Nous avons donc pu déterminer des fonctions satisfaisant aux conditions que nous nous étions imposées et nous avons réalisé une véritable généralisation des solutions périodiques. Seulement, tandis que les séries qui définissent les solutions périodiques sont convergentes, il n'en est plus de même de celles dont nous venons de démontrer l'existence, de sorte que cette généralisation n'a de valeur qu'au point de vue du calcul formel ${ }^{100}$

This divergence is crucial for the dynamics; we shall come back to this point in section 10.1 .

\footnotetext{
${ }^{100}$ We have thus been able to determine functions which satisfy the conditions we had imposed and we have accomplished a true generalization of the periodic solutions. But, while the series defining the periodic solutions are convergent, this is no more true of those which we have just proved to exist, so that this generalization has value only from the point of view of formal computation.
} 
Being mainly interested in the sequel in the Restricted Problem, I shall not discuss the degenerate case which occurs for the Non-Restricted Three-Body Problem. But, without surprise, Poincaré studies it in depth in chapter XXI. Arguing that the Lindstedt series still exist in this case, he says:

On peut donc prévoir que la méthode de M. Bohlin est encore applicable aux cas où $F_{0}$ ne dépend pas de toutes les variables de la première série et, en particulier, au problème des trois Corps. Mais l'application soulève quelques questions délicates et je suis obligé d'insister.101

\section{Integral invariants and Poisson stability}

We have already seen in section 6.1 that, when studying the exponents of periodic solutions, Poincaré makes use of the symplectic structure of the equations. But it is in chapter XXII, which opens the third volume of the New Methods that he displays the full consequences of this property.

\subsection{Integral invariants as integrals of the variational equations}

Having proved the non-existence of first integrals besides the classical ones which come from the symmetries of the problem, Poincaré sees the integral invariants as an ersatz: it consists in replacing the equations of motion by the variational equations which, indeed, admit first integrals. Explained in section 242, this filiation is largely forgotten in modern expositions:

Reprenons le système

$$
\frac{d x_{1}}{X_{1}}=\frac{d x_{2}}{X_{2}}=\cdots=\frac{d x_{n}}{X_{n}}=d t .
$$

Nous pouvons former les équations aux variations correspondantes telles qu'elles ont été définies au début du Chapitre IV. Pour former ces équations, on change dans les équations (1) $x_{i}$ en $x_{i}+\xi_{i}$ et l'on néglige les carrés des $\xi_{i}$; on trouve ainsi le système d'équations linéaires

$$
\frac{d \xi_{k}}{d t}=\frac{d X_{k}}{d x_{1}} \xi_{1}+\frac{d X_{k}}{d x_{2}} \xi_{2}+\cdots+\frac{d X_{k}}{d x_{n}} \xi_{n}
$$

Il y a, entre les intégrales des équations (2) et les invariants intégraux des équations (1), un lien intime qu'il est aisé d'apercevoir.

Soit $F\left(\xi_{1}, \xi_{2}, \cdots, \xi_{n}\right)=$ const., une intégrale quelconque des équations (2). Ce sera une fonction homogène par rapport aux $\xi$, et dépendant d'ailleurs des $x$ d'une manière quelconque. Je pourrai toujours supposer que cette fonction $F$ est homogène de degré 1 par rapport aux $\xi$; car s'il n'en était pas ainsi, je n'aurais qu'à élever $F$ à une puissance convenable pour trouver une fonction homogène de degré 1 . Considérons maintenant l'expression

$$
\int F\left(d x_{1}, \cdots, d x_{n}\right)
$$

\footnotetext{
${ }^{101}$ Hence one can predict that M. Bohlin's method is still applicable to the case where $F_{0}$ does not depend on all the variables of the first series, and, in particular, to the Three-Body Problem. But the applications raises some delicate questions and I am forced to insist.
} 
je dis que c'est un invariant intégral du système (1) ${ }^{102}$

and more specifically, in section 255 :

Dans le cas des équations de la Dynamique, il est aisé de former un grand

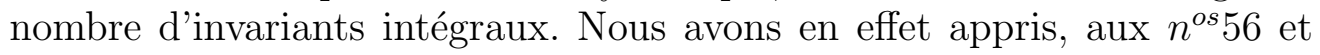
suivants, à former un certain nombre d'intégrales de l'équation aux variations et nous avons appris dans le Chapitre précédent comment on peut en déduire des invariants intégraux.

Une première intégrale (équation 3, t. I, p. 167) est la suivante

$$
\eta_{1}^{\prime} \xi_{1}-\xi_{1}^{\prime} \eta_{1}+\eta_{1}^{\prime} \xi_{1}-\xi_{1}^{\prime} \eta_{1}+\cdots=\text { const. }
$$

L'invariant intégral qu'on en déduit est le suivant

$$
J_{1}=\int\left(d x_{1} d y_{1}+d x_{2} d y_{2}+\cdots d x_{n} d y_{n}\right)
$$

Il est du deuxième ordre et fort important pour ce qui va suivre. ${ }^{103}$

Then, writing determinants which amount to computing the exterior powers of the symplectic form $d x_{1} \wedge d y_{1}+d x_{2} \wedge d y_{2}+\cdots d x_{n} \wedge d y_{n}$, he concludes :

Cependant, parmi tous ce invariants, il y en a un auquel il convient d'attacher une grande importance, c'est le dernier d'entre eux ${ }^{104}$

$$
J_{n}=\int d x_{1} d y_{1} d x_{2} d y_{2} \cdots d x_{n} d y_{n}
$$

This is of course the Liouville theorem, that is the preservation by a Hamiltonian flow of the volume in phase space. Moreover, the interior product of the symplectic form by a particular solution of the variational equation furnishes other invariants: namely, if $\xi=\varphi(t), \eta=\psi(t)$ is a solution of the variational equation, the constancy of $\xi \psi-\eta \varphi$ for any other solution $(\xi, \eta)$, implies that $\int(\psi d x-\varphi d y)$ is a (time dependent) integral invariant. In addition to the ones coming from the derivation of actual integrals of the equations of motion, Poincaré did not fail to notice that the homogeneity of the potential gives such a particular solution: if $q(t)=\left(\vec{r}_{1}(t), \vec{r}_{2}(t), \vec{r}_{3}(t)\right)$ is a solution of the Three-Body Problem, so is $\lambda^{2} q\left(\frac{t}{\lambda^{3}}\right)$ for any $\lambda>0$; taking the derivative with respect to $\lambda$ at $\lambda=1$ gives the required solution. This invariance

\footnotetext{
${ }^{102}$ Let us take back the system (1) $\frac{d x_{1}}{X_{1}}=\frac{d x_{2}}{X_{2}}=\cdots=\frac{d x_{n}}{X_{n}}=d t$. We can form the corresponding variational equations as they were defined at the beginning of Chaper IV. In order to form these equations one changes $x_{i}$ into $x_{i}+\xi_{i}$ in equations (1) and one neglects the squares of the $\xi_{i}$; one finds in this way the system of linear equations (2) $\frac{d \xi_{k}}{d t}=\frac{d X_{k}}{d x_{1}} \xi_{1}+\frac{d X_{k}}{d x_{2}} \xi_{2}+\cdots+\frac{d X_{k}}{d x_{n}} \xi_{n}$. There is, between the integrals of equations (2) and the integral invariants of equations (1), an intimate relation which is easy to see. Let $F\left(\xi_{1}, \xi_{2}, \cdots, \xi_{n}\right)=$ const., be any integral of equations (2). It is a function, homogeneous of degree 1 in the $\xi$ and depending in an arbitrary way on the $x$; indeed, if this was not the case, it would be enough to raise $F$ to a conveniently chosen power in order to obtain a function homogeneous of degree 1 . Now, let us consider the expression $\int F\left(d x_{1}, \cdots, d x_{n}\right)$, I say it is an integral invariant of system (1).

${ }^{103}$ In the case of the equations of Dynamics, it is easy to form a large number of integral invariants. Actually, we have learned in $n^{o s} 56$ and the following, to form a number of integrals of the variational equation and we have learned in the previous chapter how to deduce from them integral invariants. A first integral (equation 3, t. I, p. 167) is the following $\eta_{1}^{\prime} \xi_{1}-\xi_{1}^{\prime} \eta_{1}+\eta_{1}^{\prime} \xi_{1}-\xi_{1}^{\prime} \eta_{1}+\cdots=$ const.. The resulting integral invariant is the following $J_{1}=\int\left(d x_{1} d y_{1}+d x_{2} d y_{2}+\cdots d x_{n} d y_{n}\right)$. It is of second order and very important for what follows.

${ }^{104}$ However, among all those invariants, there is one to which one should attach a great importance, it is the last of them $J_{n}=\int d x_{1} d y_{1} d x_{2} d y_{2} \cdots d x_{n} d y_{n}$.
} 
under rescaling is at the root of the analysis of the behaviour of solutions of the three-body problem near a triple collision, first by Sundman and then more geometrically by R. McGehee thanks to the introduction of a compactification called the collision manifold (see [McG, C7]). It is only in the case of the strong force potential, proportional to the inverse square of the mutual distance that this scaling property becomes a true symplectic symmetry of the equations, implying the existence of the Jacobi integra ${ }^{105}$ (see $[\mathrm{AC}]$ ).

But there is more: in section 261, entitled "The integral invariants and the characteristic exponents", Poincaré deepens the analogy between integrals of the equations of motion and integral invariants, by asking:

On peut se demander s'il existe d'autres invariants intégraux algébriques que ceux que nous venons de former.

On pourrait appliquer, soit la méthode de Bruns, soit celle dont j'ai fait usage aux chapitres IV et V; en effet, les invariants intégraux correspondent, comme nous l'avons vu, aux intégrales des équations aux variations et l'on pourrait appliquer à ces équations les mêmes procédés qu'aux équations du mouvement elles-mêmes 106

Even if the analysis is not complete, because of the difficult control of the genericity of the periodic solutions, it has the interest of indicating probable bounds on the number of integral invariants. Poincaré concludes:

Il est probable que ces invariants nouveaux, dont la discussion précédente n'exclut pas la possibilité, n'existent pas ; mais pour le démontrer, il faudrait recourir à d'autres procédés, par exemple à des procédés analogues à la méthode de Bruns 107

To my knowledge, this has not been pursued explicitely, but see [Mal, Cas].

\section{Remarks.}

1) In the introduction of his first book Leçons sur les invariants intégraux [Ca], directly inspired by the works of Poincaré, Elie Cartan writes :

En définitive, la quantité sous le signe d'intégration dans un invariant intégral de H. Poincaré n'est autre chose qu'une forme différentielle invariante tronquée. Le caractère invariant de l'intégrale complétée est conservé si elle est étendue à un ensemble quelconque d'états, simultanés ou non ${ }^{108}$

The paradigmatic example of what Cartan calls a "completion" is the transformation of the Poincaré integral invariant $\int p d q$ into the Poincaré-Cartan integral invariant or impulsion-energy tensor $\int\left(\sum_{i} p_{i} d q_{i}-H(p, q, t) d t\right)$; both are relative invariants, which means that they have to be integrated on closed loops: the first integral does not change by transport of the loop along the flow lines, either between two fixed

\footnotetext{
${ }^{105}$ Poincaré had read Jacobi's Vorlesungen über Dynamik.

${ }^{106}$ One could ask whether there exists algebraic invariants different from those which we have just formed.

One could apply, either Bruns's method, or the one which I used in Chapters IV and V; indeed, as we saw, the integral invariants correspond to the integrals of the variational equations and one could apply to these equations the very method we applied to the equations of motion themselves.

${ }^{107}$ It is likely that these new invariants, whose possibility is not excluded by the preceding discussion, do not exist; but in order to prove it, we need to resort to other methods, e.g. processes similar to the method of Bruns.

${ }^{108}$ Ultimately, the quantity under the sign of integration in an integral invariant of $\mathrm{H}$. Poincaré is nothing but a truncated invariant differential form. The invariant character of the completed integral is preserved if it is extended to any set of states, simultaneous or not.
} 
instants or at fixed energy; the second one is invariant by any transport of the loop along graphs of flow lines in the $(q, p, t)$ space. But in fact, this was already understood by Poincaré. In section 336 which opens chapter XXIX, studying the variations of the integra ${ }^{109} J=\int_{t_{0}}^{t_{1}}\left(-F+\sum y_{i} \frac{d x_{i}}{d t}\right)$, he writes:

Nous avons supposé jusqu'à présent que les deux limites $t_{0}$ et $t_{1}$ sont données; qu'arrive-t-il si les limites sont regardées comme variables ? ?10

Supposing the Hamiltonian is autonomous and noticing that the partial derivative of the action with respect to a boundary of the time interval is, up to sign, the energy, he concludes:

Si cette constante [l'énergie] est nulle, l'action $J$ est encore minimum si l'on regarde les valeurs initiales et finales des variables $x_{i}$ comme données et quand même on ne regarderait pas comme données les valeurs initiales et finales du temps, $t_{0}$ et $t_{1}{ }^{111}$

Some pages later, in section 341, he will correct this statement in his quite charming characteristic style:

Jusqu'ici, quand j'ai dit, telle intégrale est minimum, je me suis servi d'une façon de parler abrégée, mais incorrecte, qui ne pouvait d'ailleurs tromper personne; je voulais dire, la variation première de cette intégrale est nulle; cette condition est nécessaire pour qu'il y ait minimum, mais elle n'est pas suffisante 112

Finally, recall that the property of the impulsion-energy tensor of being a relative integral invariant characterizes the equations of Hamilton (see the introduction of [Ca] or [A2]): it means that the graphs of the flow lines are tangent to the 1-dimensional kernel of the exterior derivative of $p d q-H d t$.

2) Continuing with the introduction of [Ca], one finds the strong link between the integral invariants in the sense of Poincaré and Cartan and the ones in the sense of Lie, directly relevant to the theory evoked in 6.5:

La notion d'invariant intégral peut être envisagée d'un point de vue un peu différent du point de vue habituel qui est celui de Poincaré, et qui est en somme celui où on s'est plaçé dans ces Leçons. Au lieu de considérer une intégrale multiple attachée à un système d'équations différentielles visà-vis duquel elle jouit d'une propriété d'invariance, on peut la considérer comme attachée à un groupe de transformations par rapport auquel elle est invariante. Les deux points de vue sont du reste connexes. Le dernier est celui auquel s'est placé S. Lie et qui lui a paru pendant quelque temps le seul vrai. Là encore la notion d'invariant intégral joue un rôle important puisque, comme l'auteur l'a montré [Sur la structure des groupes infinis de

\footnotetext{
109 that is, up to sign, the integral of $\sum p_{i} d q_{i}-H d t$.

${ }^{110} \mathrm{Up}$ to now, we have supposed that both limits $t_{0}$ and $t_{1}$ are given; what happens if these limits are considered as variable?

${ }^{111}$ If this constant [the energy] is equal to zero, the action $J$ is still minimum if one considers the initial and final values of the variables $x_{i}$ as given and even if one would not consider as given the initial and final values of the time, $t_{0}$ and $t_{1}$.

${ }^{112}$ So far, when I said, this integral is minimum, I used a shorthand, but incorrect, way of talking, but one which could not mislead anyone; I meaned, the first variation of this integral is zero; this condition is necessary for being a minimum, but it is not sufficient.
} 
transformations, Annales de l'E.N.S. 1904 1905], tout groupe de transformations peut, au besoin par l'adjonction de variables auxiliaires, être défini comme l'ensemble des transformations qui admettent un certain nombre d'invariants intégraux linéaires ${ }^{113}$

\subsection{Poisson stability}

Le mot stabilité a été entendu sous les sens les plus différents, et la différence de ces divers sens deviendra manifeste si l'on se rappelle l'histoire de la Science. Lagrange a démontré qu'en négligeant les carrés des masses, les grands axes des orbites deviennent invariables. Il voulait dire par là qu'avec ce degré d'approximation les grands axes peuvent se développer en séries dont les termes sont de la forme $A \sin (\alpha t+\beta), A, \alpha$ et $\beta$ étant des constantes 114

The title of chapter XXVI is not innocent. Having wrongly stated in the original version of the memoir in 1889 a very strong stability theorem in the Planar Circular Restricted Three-Body Problem, Poincaré needs a stability result which, if much weaker, will turn of considerable importance. ${ }^{115}$ Indeed, based on the recurrence theorem, it is the forerunner of ergodic theory. The Poisson stability alludes to the absence of purely secular terms (i.e., of terms which grow without limits when times goes on) in the planetary semi-major axes at the second order of the classical theory of perturbations (i.e., when neglecting the cubes of the planetary masses) which, at this order of approximation, implies a recurrent behaviour of these semi-major axes (Spiru Haret will show that this property is not shared any more by the approximations of higher orders.) Using an argument of conservation of the volume in a container of finite volume, Poincaré shows that, in the case he is considering, the conservation of the integral invariant implies that a "generic" solution of the Restricted Problem will come back infinitely often in an arbitrarily small neighborhood of a given point of the phase space. In the following quotation (chapter XXVI section 296) Poincaré legitimates his new use of the word "stability":

En résumé, les molécules qui ne traversent $U_{0}$ qu'un nombre fini de fois sont exceptionnelles au même titre que les nombres commensurables qui ne sont qu'une exception dans la série des nombres, pendant que les nombres incommensurables sont la règle. Si donc Poisson a cru pouvoir répondre affirmativement à la question de la stabilité telle qu'il l'avait posée, bien qu'il eût exclu les cas où le rapport des moyens mouvements est commensurable, nous aurons de même le droit de regarder comme démontrée la

\footnotetext{
${ }^{113}$ The notion of integral invariant can be envisaged from a somewhat different point of view of the usual one which is the one of Poincaré, and which is basically the one which we have taken in these lessons. Instead of considering a multiple integral attached to a system of differential equations with respect to which it enjoys an invariance property, it can be considered as attached to a group of transformations with respect to which it is invariant. Indeed, the two views are related. The latter is the one of S. Lie who, for a while, considered it as the only true one. Here too, the notion of integral invariant plays an important role since, as the author showed [On the structure of infinite groups of transformations, Annals of the ENS 1904 1905], any group of transformations may, after adding auxiliary variables if necessary, be defined as the set of transformations that admit some linear integral invariants.

${ }^{114}$ The word stability has been taken in very different meanings, and the difference between these meanings will become obvious if one remembers history of Science. Lagrange has proved that if one neglects the squares of the mases, the major axes of the orbits become invariable. He wanted to say that with this degree of approximation the major axes can be expanded in series whose terms are of the form $A \sin (\alpha t+\beta)$, where $A, \alpha$ and $\beta$ are constants.

${ }^{115}$ In the first version of the Memoir, Poincaré deduced this weaker stability from the actual stability that he thought he had proved.
} 
stabilité telle que nous la définissons, bien que nous soyons forcés d'exclure les molécules exceptionnelles dont nous venons de parler 116

Titled Probabilities, this section 296 has a striking visionary character: disregarding the fears of Joseph Bertrand about the "paradoxes" of continuous probabilities, Poincaré understands clearly that any choice of a regular density will lead to the same notion of negligible sets; in other words, he understands that the notion of zero measure set does not depend on such a choice:

Mais il faut d'abord que j'explique le sens que j'attache au mot probabilité. Soit $\varphi(x, y, z)$ une fonction quelconque positive des trois coordonnées $x, y, z$; je conviendrai de dire que la probabilité pour qu'à l'instant $t=0$ une molécule se trouve à l'intérieur d'un certain volume est proportionnelle à l'intégrale

$$
J=\int \varphi(x, y, z) d x d y d z
$$

étendue à ce volume. . . Nous pouvons choisir arbitrairement la fonction $\varphi$ et la probabilité se trouve ainsi complètement définie. ‥ Nous retombons donc sur les mêmes résultats qui sont ainsi indépendants du choix de la fonction $\varphi$

A beautiful analysis of this part of Poincaré's text can be found in the thesis of Anne Robadey $[\mathrm{Ro}$. See also the pleasant paper $\mathrm{G}$ to get acquainted with possible misinterpretations of the recurrence theorem.

\subsection{From the recurrence theorem to ergodic theory}

The recurrence theorem is a weak stability result but it tells us nothing on the way the solution will come back indefinitely often close to its initial point. It could be in an integrable way, staying on an invariant torus and hence exploring a very small part of the energy hypersurface or else in an ergodic way, filling a dense subset in the energy surface. The ergodic theorem makes this difference quantitatively precise. The notion of ergodicity originates from the works of Boltzmann in statistical mechanics, namely his famous theorem $H$ (1872) which asserts the monotonicity of the entropy in a system formed by a great number of interacting particles, as for example the atoms in a gaz. As noticed by Poincaré and Zermelo, this appears to be in contradiction with Poincaré's recurrence theorem. Here is what Poincaré writes in a short paper titled Le mécanisme et l'expérience, which appeared in 1893 in the Revue de métaphysique et de morale:

\footnotetext{
${ }^{116}$ Summarizing, the molecules which pass through $U_{0}$ only a finite number of times are exceptional in the same way as the rational numbers which are but an exception in the series of numbers, while the irrational numbers are the rule. Hence if Poisson considered that he could reply affirmatively to the question of the stability as he had set it, although he had excluded the case when the mean motions are commensurable, we shall have in the same way the right of considering the stability as proved, although we are forced to exclude the exceptional molecules that we have just mentioned.

${ }^{117}$ But I must first explain the meaning I give to the word probability. Let $\varphi(x, y, z)$ any positive function of the three coordinates $x, y, z$; I shall agree to say that the probability for a molecule to be at time 0 inside a certain volume is proportional to the integral$$
J=\int \varphi(x, y, z) d x d y d z
$$

extended to this volume.... We may choose arbitrarily the function $\varphi$ and the probability is then completely defined. ... So doing, we get again the same results which hence are independent of the choice of the function $\varphi$.
} 
Un théorème facile à établir nous apprend qu'un monde limité soumis aux seules lois de la mécanique, repassera toujours par un état très voisin de son état initial. Au contraire, d'après les lois expérimentales admises (si on leur attribue une valeur absolue et qu'on veuille en pousser les conséquences jusqu'au bout), l'univers tend vers un certain état final dont il ne pourra plus sortir. Dans cet état final, qui sera une sorte de mort, tous les corps seront en repos et à la même température... .

... Cet état ne sera donc pas une mort définitive de l'univers, mais une sorte de sommeil, d'où il se réveillera après des millions de millions de siècles. A ce compte, pour voir la chaleur passer d'un corps froid à un corps chaud, il ne serait plus nécessaire d'avoir la vue fine, la présence d'esprit, l'intelligence et l'adresse du démon de Maxwell, il suffirait d'un peu de patience.

On voudrait pouvoir s'arrêter à cette étape et espérer qu'un jour le télescope nous montrera un monde en train de se réveiller et où les lois de la thermodynamique seront renversées.

Malheureusement d'autres contradictions surgissent. . 118

Boltzmann replies that the time of recurrence being a priori greater than, say, the age of the solar system, this close return is never witnessed in practice for a system as complex as a gaz.

For the apparent contradiction, raised by Poincaré and Zermelo, between Boltzmann's theorem or Landau damping and the recurrence theorem, see [V] section 10. The main arguments are the infinite number of degrees of freedom and the infinite limit of the recurrrence time when the number of particles goes to infinity, the importance of the preparation of the initial condition, ...

The mathematical theory, named ergodic theory, comes to maturity in 1930, with the ergodic theorems of Von Neumann, Birkhoff and Koopman, which are strong dynamical forms of the strong law of large numbers. It goes beyong Poincaré's recurrence theorem in that it makes precise the relation between averages in the phase space and time averages in a dynamical system preserving a measure whose total mass is finite (which one can normalize to a probability measure, that is one with total mass equal to 1). More precisely, a system is said to be ergodic if the measure of any invariant subset is necessarily equal to 0 or 1 , and a consequence of Birkhoff's ergodic theorem is that the mean recurrence time of almost every point in a measurable subset $A$ is inversely proportional to the measure of $A$. A good introduction is the little book by Sinai [Sin].

\footnotetext{
${ }^{118} \mathrm{~A}$ theorem, easy to establish, tells us that a bounded world, only subject to the laws of mechanics, will always revert to a state very close to the original one. Instead, according to the admitted experimental laws (if one assigns to them an absolute value and if we want to push through the consequences), the universe tends toward a final state from which it will not be able to escape. In this final state, which is a kind of death, all bodies are at rest and at the same temperature...

... This condition will not be final death of the universe, but a kind of sleep, from which it will wake up after millions of millions of ages. If so, in order to see the heat move from a cold body to a hot body, it would no longer be necessary to have the sharp view, the presence of mind, the intelligence and the address of Maxwell's demon, a little patience would suffice.

One would wish to be able to stop at this point and hope that, one day, the telescope shows us a world waking up and where the laws of thermodynamics will be overturned.

Unfortunately other contradictions arise....
} 


\section{Stroboscopy 1) Planar Circular Restricted Three-Body Problem}

\subsection{The simplest Three-Body Problem}

En ce qui concerne le problème des trois corps, je ne suis pas sorti du cas suivant: Je considère trois masses, la première très grande, la seconde petite mais finie, la troisième infiniment petite; je suppose que les deux premières décrivent un cercle autour de leur centre de gravité commun et que la troisième se meut dans le plan de ces cercles. Tel serait le cas d'une petite planète troublée par Jupiter, si l'on négligeait l'eccentricité de Jupiter et l'inclinaison des orbites ${ }^{119}$

This vivid description of the so-called Planar Circular Restricted Three-Body Problem in the planetary case introduces the main theme of the memoir On the ThreeBody Problem and the equations of Dynamics which, as we already mentioned, wins in 1889 the prize of the King of Sweden, and makes its 35 years old author known in large circles. The small parameter $\mu$ is ratio of the mass of Jupiter to the mass of the Sun, that is approximately 1/1000. Similar equations rule the motions of the couple Earth-Moon perturbed by the Sun, the small parameter being now the ratio of the distance Earth-Moon to the distance Earth-Sun, that is approximately 1/400. G.W. Hill's works on this last problem strongly influenced Poincaré. In a rotating frame fixing the couple Sun-Jupiter in the planetary case, the couple Earth-Sun in the Lunar case, the equations have the same canonical form as above, $F$ being now the Jacobi constant. With the geodesic flow on an almost spherical surface, which Poincaré studies at the end of his life, these are natural examples of non-integrable Hamiltonian systems with $N=2$ degrees of freedom.

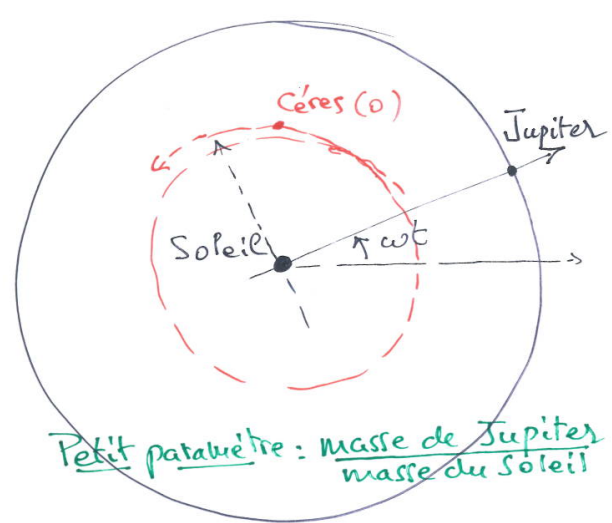

PLANETARY CASE

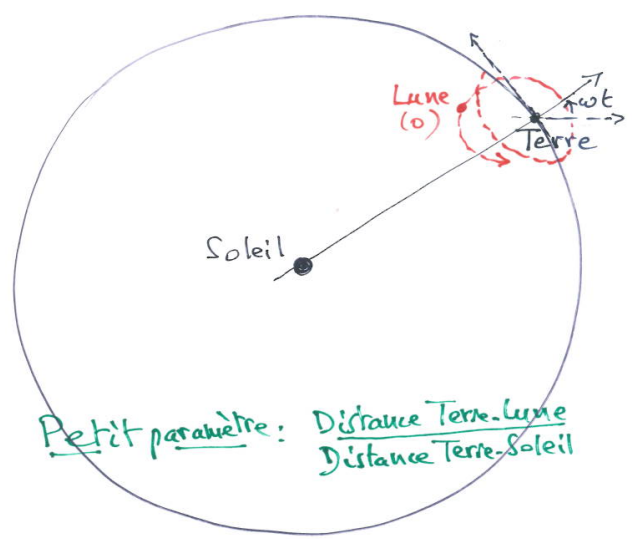

LUNAR CASE

Figure 10: The Restricted Three-Body Problem.

In canonical heliocentri ${ }^{120}$ coordinates, the Hamiltonian (= Jacobi constant) takes the form

$$
F(L, G, l, g)=-\frac{K}{L^{2}}+G+\mu F_{1}(L, G, l, g-t),
$$

\footnotetext{
${ }^{119}$ Concerning the Three-Body Problem, I did not venture away from the following case: I consider three masses, the first one very big, the second one small but finite, the third one infinitesimal; I suppose that the two first describe a circle around their common center of mass and that the third moves in the plane of these circles. Such would be the case of a small planet disturbed (I hope this word may convey the charm of the eighteen century flavoured "troublé") by Jupiter if one was neglecting Jupiter's eccentricity and the inclination of the orbits.

120 in the planetary case, canonical geocentric in the lunar case.
} 
where $K$ is a constant and $g-t$ is the argument of the perihelion of the Keplerian ellipse in the frame which rotates with angular velocity 1 (as assumed by Poincaré).
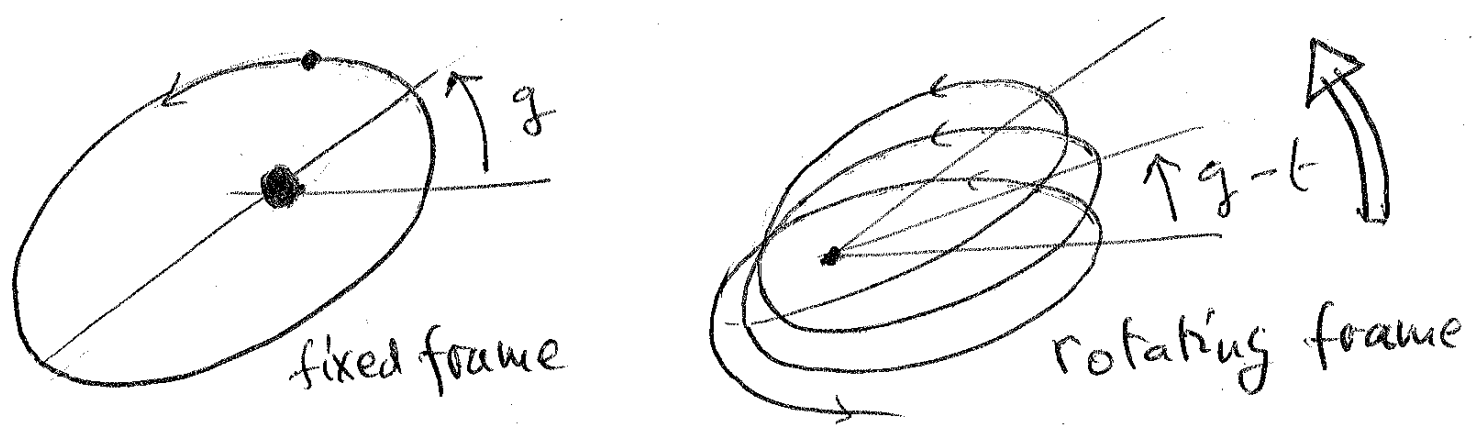

Figure 11

One nice feature is that, if still degenerate (the Hessian of the integrable part $F_{0}(L, G)=\frac{K}{L^{2}}+G$ vanishes), $F$ is easily transformed into a non-degenerate Hamiltonian by Poincaré's trick of replacing it by its exponential expF, which has the same integrability and stability properties. This replacement affects only the law of time; in particular,

the return map (see below section 9.3) is the same and hence non-degenerate.

Finally, as we already said, Delaunay coordinates are not defined in the neighborhood of circular motions and Poincaré replaces them by

$$
x_{1}=L+G, x_{2}=L+G, y_{1}=\frac{1}{2}(l-g+t), y_{2}=\frac{1}{2}(l+g-t),
$$

which are in reality 2-1 (a fact noticed by Poincaré in the Memoir but forgotten by him in The New Methods). A last step, considering these as symplectic polar coordinates, leads to the Poincaré coordinates (see section 2.2$) \sqrt{2 x_{1}} e^{i y_{1}}, \sqrt{2 x_{2}} e^{i y_{2}}$, which are regular at the circular direct or retrograde Kepler orbits. There are still problems near the collision orbits but Poincaré is only interested in the neighborhood of the circular ones.

\subsection{Hill stability}

Stability is the main question addressed by Poincaré in his Memoir (but not the main question asked for the prize, which was to find convergent series expansions of the coordinates of the bodies in case no collision occurs [BG]).

For the 2 degrees of freedom Restricted Problem, this is a question a priori simpler than for the general Three-Body Problem (see the paragraphs on K.A.M. and diffusion). Indeed, Hill had already proved that some kind of stability ocurs when the Jacobi constant is large enough, as shown on Figure 12 where an energy hypersurface and its projection on the configuration space (delimiting the so-called Hill's regions) are drawn, for such values of the Jacobi constant: escape of the infinitesimal mass from the Hill region is impossible but collisions with the closest primary are not excluded. 


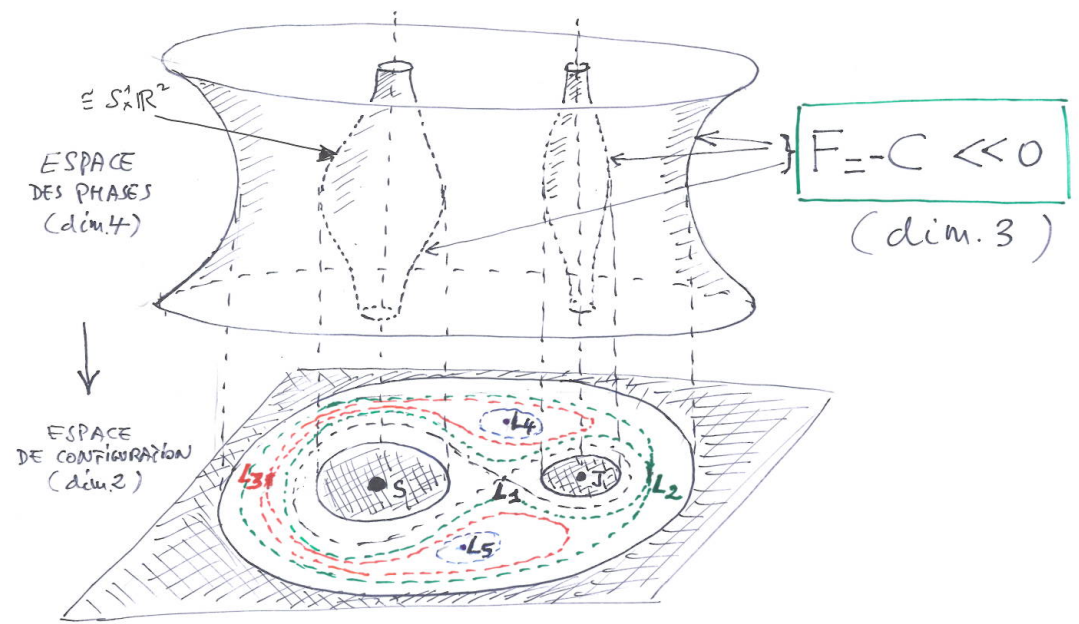

Figure 12: Hill stability.

\subsection{Return map}

In a rotating frame, the planar circular Three-Body Problem becomes an autonomous Hamiltonian system with two degrees of freedom. Fixing the energy (= Jacobi constant) to a high enough value and keeping only the part of the three-dimensional energy hypersurface above the bounded Hill region around the Sun (planetary case) or the Earth (Lunar case), one gets after regularization of the collisions, ${ }^{121}$ a manifold diffeomorphic to $S O(3)$, that is to the real projective space $\mathbb{R} P^{3}$. Poincaré writes down explicit coordinates in the 2-fold covering, diffeomorphic to $S^{3}$ of such a hypersurface deprived of one point, which makes it diffeomorphic to $\mathbb{R}^{3}$.

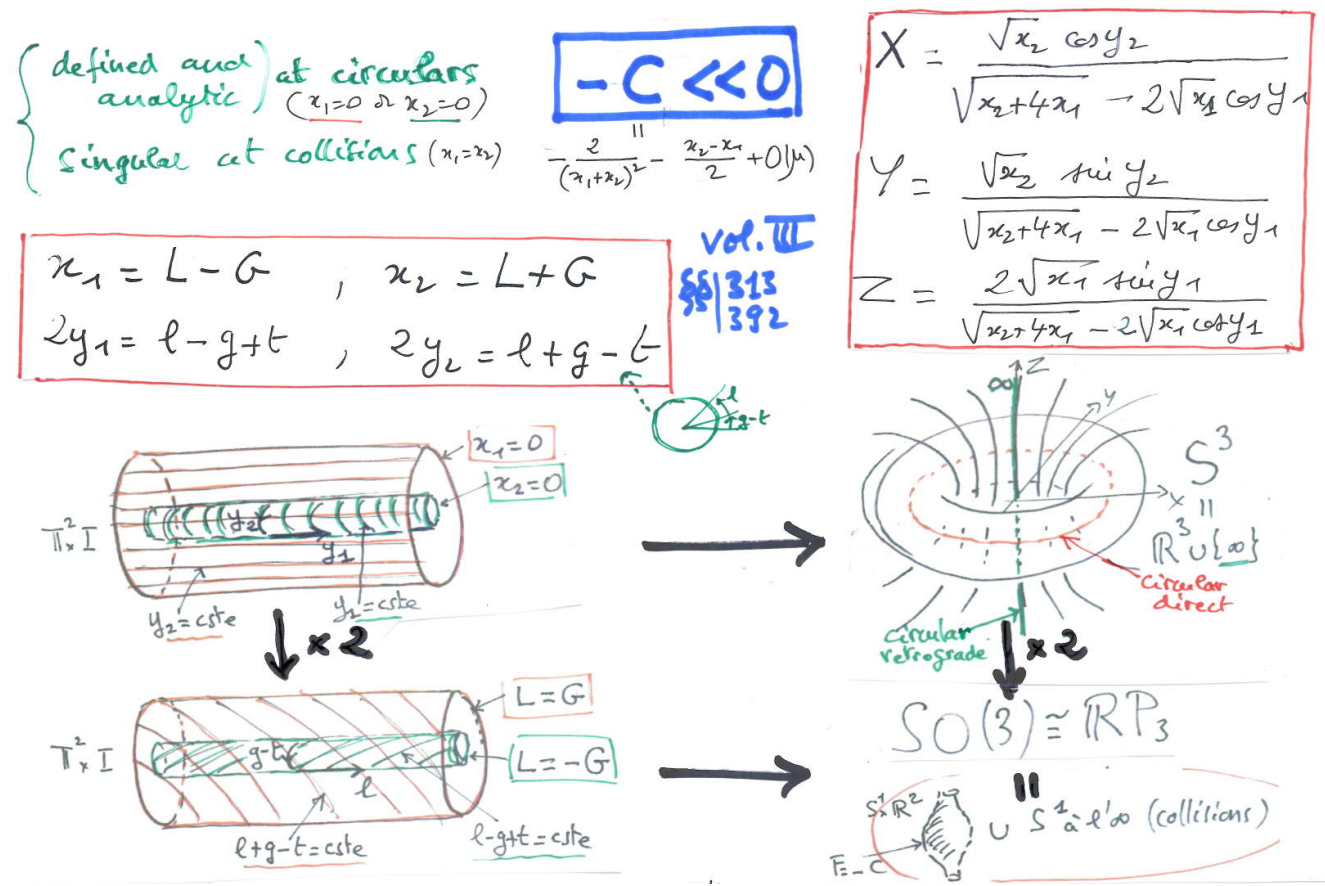

Figure 13: Coordinates in the compactified energy surface.

\footnotetext{
${ }^{121}$ The regularization, due to Levi-Civita $[\mathrm{LC}]$ (using a complex transformation anticipated by Goursat Go] in the same year 1889 as the printing of Poincaré's Memoir), amounts to compactifiying the energy submanifold by the addition of a circle corresponding to every possible direction of the velocities of the colliding bodies (see Co ).
} 
Being interested only in motions of the 0-mass body close to circular (direct or retrograde), Poincaré does not care about the fact that his coordinates are not smooth at collisions 122

In the section 305 of chapter XXVII, Poincaré embeds in this $\mathbb{R}^{3}$ a half-plane with the property that all integral curves, except the retrograde Hill orbit. ${ }^{123}$ cut it transversely an infinite number of times. Such a halph-plane thus becomes a stroboscope; describing the sucession $M_{0}, M_{1}, \ldots$ of points of intersection of a single trajectory, Poincaré writes:

Le point $M_{1}$ sera dit le conséquent de $M_{0}$. Ce qui justifie cette dénomination, c'est que, si l'on considère le faisceau des courbes qui satisfont aux équations différentielles (1); si, par le point $M_{0}$, on fait passer une courbe et qu'on la prolonge jusqu'à ce qu'elle rencontre de nouveau le demi-plan $(y=0, x>$ $0)$, cette nouvelle rencontre aura lieu en $M_{1}{ }^{124}$

Figure 14 shows the surfaces of section and the return maps, both in the unperturbed $\left(F_{0}(L, G)=-\frac{K}{L^{2}}+G\right)$ and perturbed $\left(F_{0}(L, G)=-\frac{K}{L^{2}}+G+\mu F_{1}(L, G, l, g-t)\right)$ cases.
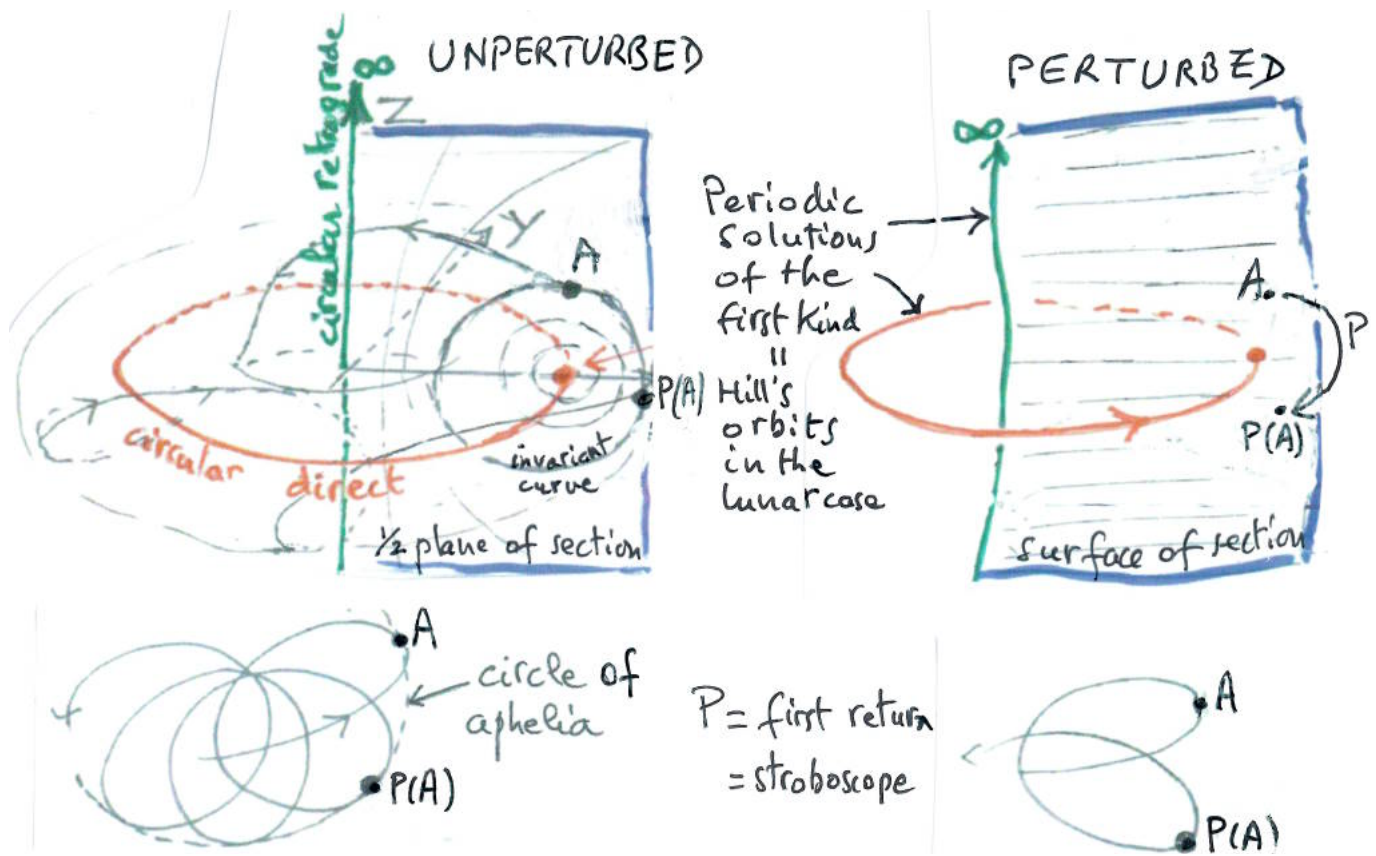

Figure 14: Poincaré retun map.

Hence the whole dynamics of the Restricted Problem with high Jacobi constant in a bounded Hill region is reduced to the study of the orbits under iteration of a mapping (the Poincaré return map) on a surface of section (here a half-plane but more accurately an annulus as described below; this return map may be loosely interpreted as describing the successive positions of the perihelium (or aphelium, which makes easier drawing, see Figure 14) of the osculating Keplerian ellipse of the zero mass body.

\footnotetext{
${ }^{122}$ because $l$ must be defined from the eccentric anomaly $u$ by Kepler formula $l=u-e \sin u$.

${ }^{123}$ The direct and retrograde Hill orbits which constitutes its boundary, are periodic solutions of the first sort which respectively continue the circular, direct and regrograde, Kepler orbit which still exist in the rotating frame. ${ }^{124}$ The point $M_{1}$ will be called the consequent of $M_{0}$. The justification of this denomination is that, if one considers the set of curves satisfying the differential equations (1); if, through the point $M_{0}$, passes a curve and if we extend this curve until it meets again the half-plane $(y=0, x>0)$, this new encounter will take place in $M_{1}$.
} 
In other words, stroboscopy in an energy manifold has turned the continuous dynamical system defined by the equations of motion into a discrete dynamical system, i.e., the continuous time into a discrete time. Doing so, one looses the time parametrization of the solutions but not their asymptotic behaviour: periodic solutions become periodic orbits of the return map, quasi-periodic solutions dense in invariant tori become quasi-periodic orbits dense in invariant curves, ... Finally, the question of stability can be wholly understood on the return map.

Remark. Topologically, it is nicer to put the direct and retrograde Hill orbits on equal footing, and work in the Birkhoff annulus of section depicted on Figure 15. It is obtained from Poincaré half-plane by blowing up the fixed point corresponding to the direct Hill orbit and adding the missing point on the retrograde Hill orbit. It is embedded in $S^{3}$, which is a two-fold cover of the regularized energy manifold; it cuts transversely every solution, except the Hill orbits which are its boundary. Actually, the flow lines defined in a regularized energy surface by the (non-rotating) Kepler Hamiltonian $F_{K e p}=-\frac{K}{L^{2}}$ coincide with the fibers of a Hopf fibration: $S^{3} \rightarrow S^{2}$, where the basis is the space of oriented ellipses (possibly degenerate) with a fixed value of the semi-major axis. Blowing up two fibers (here the Hill orbits) is the simplest ersatz of a global section for this non-trivial fiber bundle. For more details, see [Co, C5]

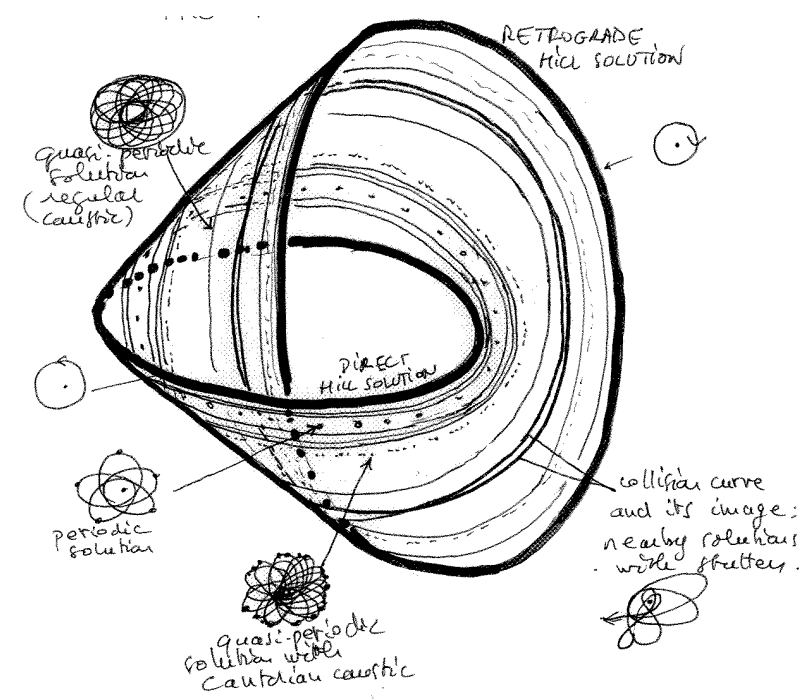

Figure 15: Birkhoff's annulus of section.

\section{Resonances 2) Homoclinic and heteroclinic tangles}

\subsection{Divergence of the Bohlin series and exponentially small splitting}

Let us stick to the Restricted Problem. The periodic solutions of the second sort result from the breaking of invariant tori of the unperturbed system $F_{0}$ filled with periodic solutions. In the surface of section, they correspond to periodic orbits bifurcating from invariant curves of the unperturbed return map filled with periodic orbits; they are in general born in pairs elliptic-hyperbolic when the parameter $\mu$ takes a non-zero value. At first order, the formal Bohlin series describe separatrices of the hyperbolic ones forming penduli and it was precisely the error of Poincaré to have considered these penduli as real (i.e., the Bohlin series to be convergent) 
and hence separating in the three-dimensional energy hypersurface. In the corrected version of the Memoir and in the second and third volume of the New Methods, Poincaré studies the actual phenomenon: if the stable and unstable invariant manifolds coincide formally (i.e., at any order of the theory of perturbations), as shown by the existence of formal Bohlin series in the separatrix case, they do not actually coincide in general.

In chapter XXI (volume II), Poincaré shows by a direct computation the divergence of the Bohlin series in the case of a periodically forced pendulum. The equation is turned into a two degrees of freedom Hamiltonian by introducing the symplectic pair of energy-time $(E, \tau)$ coordinates:

$$
\mathcal{F}(x, E, y, \tau)=E+F(x, y, \tau), \quad F(x, y, \tau)=x^{2}-2 \mu \sin ^{2} \frac{y}{2}-\mu \epsilon \varphi(y) \cos \tau .
$$

The same trick of decoupling the pendulum term and the perturbation term by the introduction of a second parameter will be used by Arnold in his famous example of diffusion [A4 to overcome the so-called "big gap" problem. For $\epsilon=0$, the $2 \pi$ periodic solution $x=E=0, y=0, \tau=t$ has asymptotic solutions of the form

$$
E=0, x= \pm \sqrt{2 \mu} \sin \frac{y}{2}, \tan \frac{y}{4}=C e^{ \pm t \sqrt{2 \mu}} \text {. }
$$

Seeking solutions $S(y, \tau)=S_{0}+\epsilon S_{1}+\epsilon^{2} S_{2}+\cdots$ of the Hamilton-Jacobi equation

$$
F\left(\frac{\partial S}{\partial y}, y, \tau\right)=C=\epsilon C_{1}+\epsilon^{2} C_{2}+\cdots
$$

we find

$$
S_{0}=\mp 2 \sqrt{2 \mu} \cos \frac{y}{2}
$$

while $S_{1}$ must satisfies the equation

$$
\frac{\partial S_{1}}{\partial \tau}+\frac{\partial F_{0}}{\partial x}\left(\frac{\partial S_{0}}{\partial y}\right) \frac{\partial S_{1}}{\partial y}-\mu \varphi(y) \cos \tau=C_{1}
$$

that is

$$
\frac{\partial S_{1}}{\partial \tau}+\left(2 \sqrt{2 \mu} \sin \frac{y}{2}\right) \frac{\partial S_{1}}{\partial y}-\mu \varphi(y) \cos \tau=C_{1},
$$

which is easily integrated by variation of the constants. Writing $S_{1}$ as the real part of a complex function and choosing complex paths to compute the relevant integrals, Poincaré finds two solutions $S_{1}(y, \tau)$ and $S_{1}^{\prime}(y, \tau)$ with the property that $S_{1}$ vanishes for $y=2 \pi$ and $S_{1}^{\prime}$ vanishes for $y=0$. Moreover, he evaluates the difference $S_{1}^{\prime}-S_{1}$ by a simple residue computation; in the case where $\varphi(y)=\sin y$, the computation is explicit; it shows that

$$
S_{1}^{\prime}-S_{1}=O\left(\frac{1}{\sqrt{\mu}} e^{-\frac{\pi}{4 \sqrt{2 \mu}}}\right)
$$

while exponentially small with respect to $\mu$, is different from 0 , which shows the divergence of the Bohlin series obtained by expanding $S$ in powers of $\sqrt{\mu}$. For an interpretation in terms of the so-called Poincaré-Melnikov integral whose non vanishing is equivalent to the non vanishing of $S_{1}^{\prime}-S_{1}$, see for example the chapter 7 of $\mathrm{AKN}$. 
At the very end of the second volume of The New Methods, making the link with his study of the asymptotic solutions in chapter VII (see section 6.1), Poincaré gives a more geometric interpretation of this exponentially small difference in terms of the stable and unstable manifolds of the family of unstable (hyperbolic) periodic solutions which are the continuation of the one for $\epsilon=0$. These invariant manifolds do not coincide but they share a common formal asymptotic expansion in powers of $\sqrt{\mu}$ and this expansion is precisely the Bohlin series. For a thorough study of this example in the light of Ecalle's theory of resurgent functions, see [Sau]; for a more general survey, see Ra2].

\section{2 "... cette figure, que je ne cherche même pas à tracer"}

Poincaré's mistake in the first version of the Memoir (see [BG, Y]) was asserting that the Bohlin series did converge and hence that these asymptotic curves formed penduli and hence true barriers to the dynamics:

Donc les surfaces asymptotiques sont des surfaces fermées. Mais au début de ce travail, nous avons montré que pour établir la stabilité, il suffit de démontrer l'existence de surfaces trajectoires fermées ${ }^{125}$

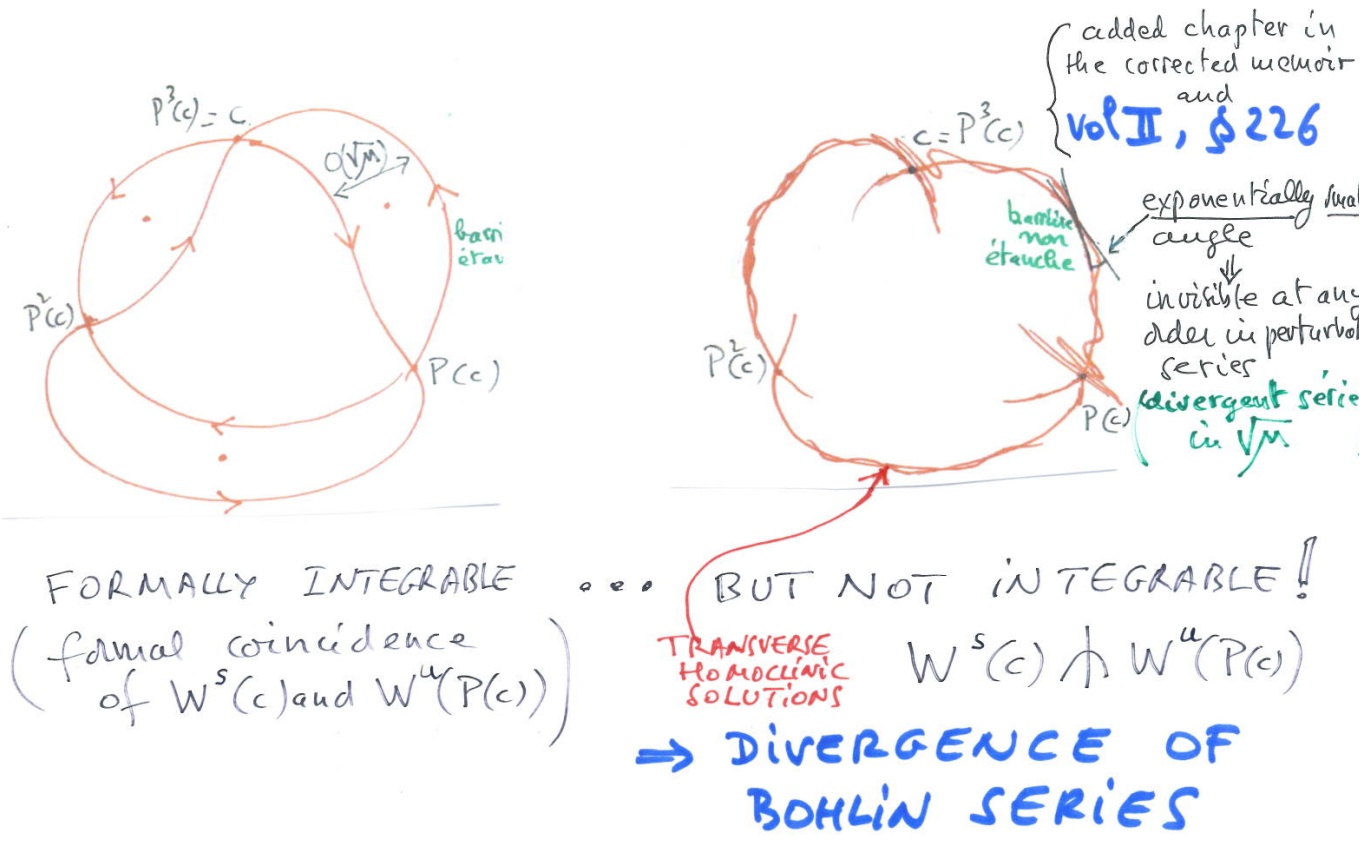

Figure 16: The error in the first version of the Memoir.

The discovery by Poincaré of the geometrical complexity caused by the non-convergence of the Bohlin series shapes our present understanding of the qualitative behaviour of solutions of a differential equation. In the following description, I combine chapters XXVII and XXXIII. Here are the main steps:

1) Showing that the preservation of the symplectic form implies the preservation by the return map of a measure with a smooth density with respect to Lebesgue

\footnotetext{
${ }^{125}$ Hence the asymptotic surfaces are closed surfaces. But at the beginning of this work, we have shown that in order to establish the stability, it is enough to prove the existence of closed surface-trajectories.
} 
and finite total mass ${ }^{126}$ This is done by using explicit formulæ in the coordinates described in Figure 13.

2) Proving that the asymptotic curves which are the intersection with the surface of section of the stable and unstable manifolds of the newborn periodic solutions must intersect (in Figure 17: $A_{0} A_{5}$ and $B_{0} B_{5}$ must intersect); the argument is a beautiful combination of invariance of the measure, topology of the plane and clever use of the fact that the asymptotic curves are exponentially close with respect to the parameter in a certain region, more precisely, that the segments $A_{0} B_{0}$ and $A_{5} B_{5}$ in Figure 17 are exponentially small.

\begin{abstract}
I94 CHAPITRE XXVII.
J'ai fait la figure en supposant, pour fixer les idées, $m_{1}=5$, $m_{2}=2$. La trajectoire fermée $\mathrm{T}$ coupe le demi-plan aux cinq points $\mathbf{M}_{0}, \mathbf{M}_{1}, \mathbf{M}_{2}, \mathbf{M}_{3}, \mathbf{M}_{4}$. Par cette trajectoire passent deux surfaces asymptotiques qui se coupent.

I'intersection de ces surfaces asymptotiques avec le demi-plan se composera de diverses courbes; nous aurons deux courbes se coupant en $\mathbf{M}_{0}$, deux en $\mathbf{M}_{1}$, deux en $\mathbf{M}_{2}$, deux en $\mathbf{M}_{3}$, deux en $\mathbf{M}_{4}$. Toutes ces courbes sont représentées sur la figure.
\end{abstract}

Fig. 7.

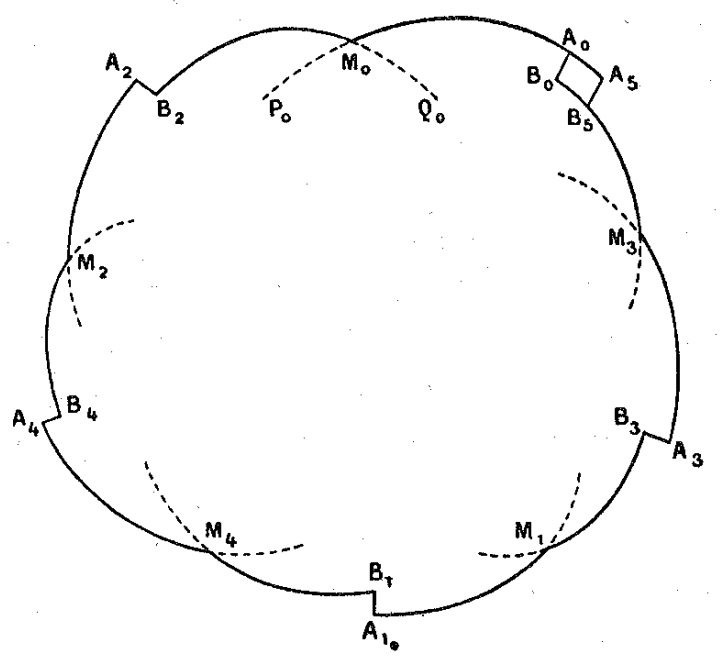

Figure 17: The intersection property (this situation cannot occur).

The upshot is that the asymptotic curves must intersect and at the intersection points, they make exponentially small angles. It seems at first sight that piecing together segments of asymptotic curves, one defines closed curves which could form barriers implying stability of the dynamics as penduli would have done (see Figure 16) but it is not so. Such curves are not invariant and if iterated, they become an incredibly complicated tangle which is not an hermetic barrier but which nevertheless slows down the dynamics: one can get through but only very slowly, hence there is a priori no stability but the instability needs a very long time to be detectable.

\footnotetext{
${ }^{126}$ Of course, Poincaré does not use these words because Measure theory does not exist yet.
} 
Understanding the extreme complexity of the intersections of these two asymptotic manifolds which must fold and stretch more and more when approaching the periodic point, Poincaré writes (one shoud rather say "exclaims"):

Que l'on cherche à se représenter la figure formée par ces deux courbes et leurs intersections en nombre infini dont chacune correspond à une solution doublement asymptotique, ces intersections forment une sorte de treillis, de tissu, de réseau à mailles infiniment serrées ; chacune des deux courbes ne doit jamais se recouper elle-même, mais elle doit se replier sur elle-même d'une manière très complexe pour venir recouper une infinité de fois toutes les mailles du réseau.

On sera frappé de la complexité de cette figure, que je ne cherche même pas à tracer. Rien n'est plus propre à nous donner un idée de la complication du problème des trois corps et en général de tous les problèmes de Dynamique où il n'y a pas d'intégrale uniforme et où les série de Bohlin sont divergentes. ${ }^{127}$ (chapitre XXXIII, section 397).

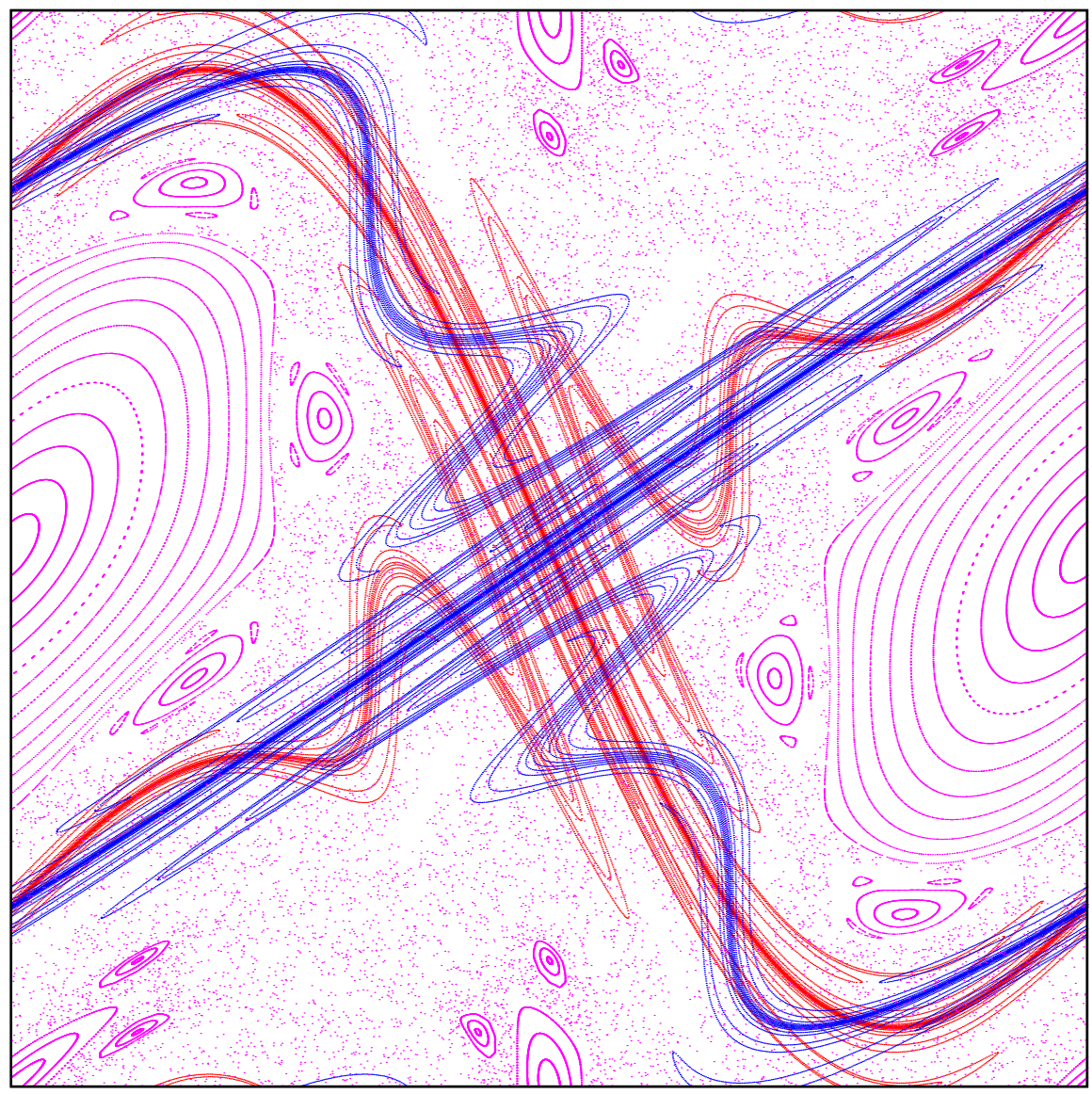

Figure 18: A tangle computed by Carles Simó.

\footnotetext{
${ }^{127}$ Let us try to represent the figure formed by these two curves and their intersections in infinite number, each corresponding to a doubly asymptotic solution, these intersections form a kind of mesh, of fabric, of infinitely tight network; each of the two curves must never intersect itself, but it must fold back on itself in a very complex way in order to cross an infinite number of times all the meshes of the network.

One will be struck by the complexity of this figure, which I do not even try to draw. Nothing is more likely to give us an idea of the complexity of the Three-Body Problem and in general of all the problems of dynamics where there is no uniform integral and where the Bohlin series are divergent.
} 
At the origin of that what some have called (in my opinion, quite inappropriately) "chaos theory", the complexity of the lattice of homoclinic or heteroclinic intersections of stable and unstable manifolds was partly analysed by Smale (see [Sm1, Sm2, C0]), thanks to the methods of symbolic dynamics developed by Hadamard, G.D. Birkhoff, M. Morse, G. Hedlund, ... But, even if the computers give a refined picture (as in Figure 18), understanding all the detals remains difficult.

Now comes a question: what is the relation between the divergence of Lindstedt series and the one of Bohlin series? Does the abundance of Fourier coefficients of high order imply both divergences, which would make the link, emphasized by Ramis in [Ra1, between divergence, non-integrability and ambiguity stronger? The proof of non-integrability given in volume I and the proof of the divergence of Lindstedt series which is a consequence, are based on the breaking of periodic tori which gives rise to hyperbolic (i.e., with non-zero exponents) periodic orbits. The splitting of separatrices, linked to the divergence of the Bohlin series, plays no role in this proof. Would it be possible that in a system similar to the Three-Body system but more degenerate, the analogue of the Lindstedt series would diverge while the analogue of the Bohlin series would converge? Looking at the complex singularities of the equations of separatrices this looks unlikely but how to prove it? A way to reformulate the question is asking whether Poincaré could have deduced the divergence of the Bohlin series from the divergence of the Lindstedt series.

Finally, the error of the first version of the Memoir was extraordinarily fruitful. Phragmen's questions led Poincaré to discover one of the prominent features not only of the non-integrable Hamiltonian systems, but also more generally of many dissipative generic systems. In his study of the way Poincaré was dealing with divergent series, J.P. Ramis Ra1, besides explaining how much of the present studies on resummation of divergent series was foreseen by Poincaré, rightly insists on the fact that the discovery of the homoclinic tangle due to the splitting of the separatrices turns the divergence of the series into a positive phenomenon generating a whole geometry. For a first approach, in the non conservative case, of such structures, in particular Smale's horseshoø ${ }^{128}$ and its connexion with the game of heads and tails, that is in mathematical terms with the Bernouilli shift and symbolic dynamics, I refer to [C]. For an example of analysis of symbolic dynamics type in a Three-Body Problem (namely the Sitnikov problem) see the beautiful book by Jurgen Moser [M5]. For a survey of some symbolic dynamics analysis of complicated motions in the planar Three-Body Problem associated with homoclinic and heteroclinic phenomena near triple collision, see the not less beautiful paper by Rick Moeckel [Moe]. Finally, it may seem appropriate to conclude this part with a sentence by René Thom in [Th] page 132:

Ce qui limite le vrai, ce n'est pas le faux, c'est l'insignifiant 129

\footnotetext{
${ }^{128}$ The horseshoe was not discovered at first in the homoclinic tangle. Smale explains that he discovered this paradigmatic model while trying to understand geometrically a paper by Cartwright and Littlewood which showed that a dynamical system could have persistently an infinite number of periodic orbits. This refuted the conjecture he had made that generically a dynamical system would have only a finite number of such orbits. Note that if true, this would have been a complete contrast with the conservative case and the dream of Poincaré that maybe periodic orbits could be generically dense in the phase space.

${ }^{129}$ That which limits the true is not the false, it is the insignificant.
} 


\section{Quasi-periodic solutions 2) Analytic aspects: K.A.M. stability}

\subsection{New methods, chapter XIII, section 149}

It is in the famous section 149 of chapter XIII that Poincaré addresses the issue of convergence, distinguishing the series "with variable frequencies" from the ones with "fixed frequencies". For the first ones, the divergence originates from the "generic" behaviour of the periodic solutions (a priori, but not a posteriori, independently of the non-integrability), About the second ones, he writes:

Il nous reste à traiter la deuxième question; on peut encore, en effet, se demander si ces séries ne pourraient pas converger pour les petites valeurs de $\mu$, quand on attribue aux $x_{i}^{0}$ certaines valeurs convenablement choisies ... Supposons, pour simplifier, qu'il y ait deux degrés de liberté; les séries ne pourraient-elles pas, par exemple, converger quand $x_{1}^{0}$ et $x_{2}^{0}$ ont été choisis de telle sorte que le rapport $\frac{n_{1}}{n_{2}}$ soit incommensurable, et que son carré soit au contraire commensurable (ou quand le rapport $\frac{n_{1}}{n_{2}}$ est assujetti à une autre condition analogue à celle que je viens d'énoncer un peu au hasard)?

Les raisonnements de ce Chapitre ne me permettent pas d'affirmer que ce fait ne se présentera pas. Tout ce qu'il m'est permis de dire, c'est qu'il est fort invraisemblable 130

The existence of solutions of equations (1) which are quasi-periodic with frequencies satisfying Diophantine conditions was announced ${ }^{131}$ for the first time by A. N. Kolmogorov in $1954[\mathrm{~K}$ ] in the non-degenerate case where the unperturbed Hamiltonian depends effectively on all the actions. Kolmogorov's proof was written by V. I. Arnold [A3] and extended by him to degenerate cases of the type of the planar Three-Body Problem [A1]; a different proof, suitable to the differentiable (i.e., non analytic) case was given by J. Moser [M2], hence the acronym "K.A.M. theory" (see AKN); Finally, the convergence of Lindstedt series with Diophantine fixed frequencies ${ }^{132}$ which follows if one knows the analytic dependence of these solutions with respect to the parameter $\mu$, was proved by Moser in [M2]. The invariant tori have a Whitney $C^{\infty}$ dependence on the frequencies [Laz, Pos]; asked by Kolmogorov, their monogenic dependence on a complex domain containing the Diophantine frequencies has been proved in the particular case of the Standard map (see [CMS]).

Kolmogorov's theorem or Moser's invariant curve theorem apply directly to the Planar Circular Restricted Three-Body Problem to prove the existence of a Cantor set of 2-dimensional invariant Lagrangian tori in each 3-dimensional fixed energy submanifold or equivalently a Cantor set of invariant curves of the Poincaré return map in a surface of section as defined in section 9.3. For the (non restricted) ThreeBody Problem, the degeneracy of the Kepler Problem seriously complicates the situation.

\footnotetext{
${ }^{130}$ It remains to deal with the second question; we can still, in fact, ask whether the series can not converge for small values of $\mu$, when the $x_{i}^{0}$ are given certain suitably chosen values ..... Suppose, for simplicity, that there are two degrees of freedom; could, for example, the series converge when $x_{1}^{0}$ and $x_{2}^{0}$ have been chosen so that the ratio $\frac{n_{1}}{n_{2}}$ is non commensurable, and its square is on the contrary commensurable (or when the ratio $\frac{n_{1}}{n_{2}}$ is subject to a condition similar to the one I have just stated somewhat at random? The arguments of this chapter do not allow me to affirm that such a case will not arise. All I can say is that it is highly unlikely.

131 and, according to Sinai, fully proved in his seminar.

${ }^{132}$ Recall (see 5.6 that such a convergence is possible only in the non-degenerate case.
} 


\subsection{Arnold's theorem for the planar problem}

Arnold's extension [A1] in 1963 of Kolmogorov's theorem was a major breakthrough ${ }^{133}$ in the Three-Body Problem, even if applying only to microscopic values of the planetary masses: for the first time, one had a rigourous proof of the existence in the phase space of the planar Three-Body Problem of a set of positive measure of quasiperiodic motions similar to the ones existing in the completely integrable secular system near its singularity, namely, motions of the planets along almost circular and almost coplanar ellipses slowly precessing along the centuries and whose semi-major axes, eccentricities and inclinations remain close to their initial values.

The strategy is close to Poincaré's strategy for constructing Lindstedt series: using the fact that far from mean motion resonances, the averaged system provides a good approximation to the actual system, Arnold reasons as follows: consider the completely integrable system obtained by averaging the fast angles and truncating at fourth order the Birkhoff normal form ${ }^{134}$ of the secular part at the singularity corresponding to circular and horizontal motions. The Hamiltonian, defined on a subset of $\mathbb{R}^{2} \times \mathbb{T}^{2} \times \mathbb{C}^{2}$ (coordinates $\tilde{\Lambda}, \tilde{\lambda}, \tilde{u}=\left(\tilde{u}_{1}, \tilde{u}_{2}\right)$ ), is of the form

$$
\begin{aligned}
H & =H_{0}(\tilde{\Lambda})+\mu H_{1}(\tilde{\Lambda}, \tilde{u}), \\
H_{1} & =\sum_{1 \leq j \leq 2} A_{j}(\tilde{\Lambda})\left|\tilde{u}_{j}\right|^{2}+\sum_{j, k=1}^{2} B_{j k}(\tilde{\Lambda})\left|\tilde{u}_{j}\right|^{2}\left|\tilde{u}_{k}\right|^{2} .
\end{aligned}
$$

The fast actions $\tilde{\Lambda}$ and the slow ones $\left|\tilde{u}_{j}\right|^{2}$ are first integrals of this system and fixing them defines 4-dimensional Lagrangian invariant tori in the 8-dimensional phase space. Recall (see 5.6) that the restriction of the flow of the averaged system to the subset defined by the condition that $\tilde{\Lambda}$ avoids a neighborhood of the secular set $\mathcal{S}$, can be embedded in the phase space of the planetary problem so as to approximate the actual flow. Provided the frequencies of the invariant tori of this integrable approximation depend in a sufficiently non-degenerate way on the actions, that is if

$$
\operatorname{det} \frac{\partial^{2} H_{0}}{\partial \tilde{\Lambda}^{2}}(\tilde{\Lambda}) \neq 0 \quad \text { and } \quad \operatorname{det} B_{j k} \neq 0
$$

(one then says that there is torsion) one can apply refined KAM techniques to prove the persistence - if $\mu$, that is the planetary masses, is small enough and we stay close enough ${ }^{135}$ to the secular singularity i.e., if the eccentricities and inclinations are small enough - of "sufficiently non resonant" invariant tori of the averaged system. The first determinant is easily seen to be different from zero. For the second one, Arnold checks this in the limit of a vanishing ratio of the semi-major axes and argues that analyticity grants the non vanishing of the determinant except for isolated values of this ratio. In this way, he proves the existence of quasi-periodic solutions with 4 frequencies: the two mean motions which are of order $O(1)$ and the secular frequencies of the two perihelia which are of order $O(\mu)$.

\footnotetext{
${ }^{133}$ It was also a remarkable introduction to the theory of perturbations using "the frightening formal apparatus of dynamics" and even containing some exercises.

${ }^{134}$ which exists provided one avoids a finite number of secular resonances; Arnold used the Birkhoff normal form up to order 6 but this is not necessary (see [CP]).

${ }^{135}$ but not too close, unless we center the Poincaré coordinates on a periodic solution of the first sort as explained in 5.6
} 


\subsection{Dealing with the spatial problem}

We have seen in section 5.7 that two resonances do complicate the spatial ThreeBody Problem which fortunately disappear both after complete reduction of the rotational symmetry. Arnold, well aware of the first one, $A_{4}=0$, proposed indeed to perform Jacobi's reduction (called elimination of the node because it amounts to eliminating the vertical variables) but he did not check himself accurately the presence of torsion. This was done for the first time in 1995 by Philippe Robutel [Rob], using the dedicated computer algebra system TRIP developed by Jacques Laskar LasRob to compute the torsion, and also showing theoretically that it is not zero in the limit of an infinitesimal ratio of the semi-major axes.

For an arbitrary number of planets in $\mathbb{R}^{3}$, two strategies were used in order to rigorously prove Arnold's claim in spite of the resonances (see [Chi, Fe2, Fe3] for surveys):

1) Herman and Féjoz's proof, which Herman explained in detail in his seminar during the late nineties. Due to his untimely death he could not write it fully and the proof was completed and written by Jacques Féjoz [Fe4. The main novelty was to use only the quadratic part of the secular system (i.e., the linearized system) joint with a trick, due to Poincaré and amounting to a reduction, which was to add to the Hamiltonian a small perturbation commuting with it, for example a perturbation proportional to a component of the angular momentum, in order to ensure with a minimum of computations a weak form of non degeneracy ${ }^{136}$. The existence of invariant tori followed from a beautiful normal form theorem ${ }^{137}$, subsuming the degenerate and non degenerate cases as well as the case of invariant tori of non maximal dimension. Finally, an argument of Lagrangian intersection ensured that ergodi ${ }^{138}$ invariant KAM tori of the modified Hamiltonian were still invariant by the original one (but not necessarily ergodic any more: one could just assert that they were ergodic after full reduction).

2) Chierchia and Pinzari's proof $\mathrm{CP}$ which is in a sense closer to Arnold's one. Using a modification of Delaunay coordinates due to Deprit, they notice, following MRL, that both resonances do not imped the construction of a Birkhoff normal form after the partial reduction consisting in the fixation of the direction of the angular momentum, that is restricting the $(1+n)$-body Hamiltonian to an invariant symplectic submanifold of codimension 2 (and hence dimension $6 n-2$ ) of the phase space. Restricting to such a submanifold is the natural way to reduce the non commutative symmetry group $S O(3)$ to one of its maximal tori $S O(2)$, and hence coming back in some sense to the situation of the planar problem. And indeed, the authors show the existence of torsion in the Birkhoff normal form after such a partial reduction. The Arnold KAM mechanism (more accurately a refined version) then gives ergodic invariant KAM tori of dimension $3 n-1$.

To summarize, for the spatial Three-Body Problem, one has the existence of 5-dimensional ergodic KAM tori. The five frequencies are easily identified, coming

\footnotetext{
${ }^{136}$ namely, the non planarity of the frequency map, a condition introduced by Arnold and studied by Pyartli, which is sufficient to ensure that the set of actions whose frequency is Diophantine has a positive Lebsegue measure. In agreement with Arnold's law that, in general, a theorem or a notion is not due to the person whose name it bears, this condition is usually called the Rüsmann condition.

${ }^{137}$ which was in fact already present in Moser's 1967 paper M2 in the more general setting of non necessarily conservative systems.

${ }^{138}$ More precisely "bearing linear flows with dense orbits".
} 
from the mean motions, the precessions of both perihelia and the precession of the common node in the Laplace plane (see 2.3).

Applying the rotation group, that is rotating the direction of the angular momentum, one finds invariant submanifolds of the full phase space which are diffeomorphic to the product $S^{2} \times \mathbb{T}^{5}$ of a sphere by a torus, foliated by the 5 -dimensional invariant tori: in other words, the non-commutative part of the symmetry group acts in a trivial way at the dynamical level. This is not the case of the commutative part: if one reduces completely the rotational symmetry (i.e., if one goes to the quotient by the remaining $S O(2)$-symmetry), one shall find ergodic invariant KAM tori of dimension 4 in the reduced phase space (as in Herman-Féjoz's proof). When lifted to one of the partially reduced submanifolds, one finds again 5-dimensional tori but such tori may be either ergodic or present a resonance between the reduced flow and the rotation 139

\section{Stroboscopy 2) What we understand of the dynamics of the return map}

\subsection{Bifurcations: subharmonics}

All of Poincaré's studies of periodic solutions show a remarkable insight which prefigure the theory of singularities and the studies of bifurcations. He proceeds in general from the "general case" to more and more degenerate ones ${ }^{140}$ and he does not hesitate mixing quantitative and qualitative tools. For example, in order to be sure that the solutions exist he uses the fact that a zero of the derivative at a maximum point of a holomorphic function is necessarily of odd order, relying for that on a theorem proved at the beginning of his thesis which is exactly the Weirerstrass preparation theorem ${ }^{141}$ He then can apply the result of an earlier paragraph asserting that a zero of odd order cannot disappear under small perturbation. Here is a typical sentence, in section 37 :

Une solution périodique ne peut donc disparaître qu'après s'être confondue avec une autre solution périodique.

En d'autres termes, les solutions périodiques disparaissent par couples à la façon des racines réelles des équations algébriques ${ }^{142}$

The solutions of the second kind (second genre, which are born from those of the first kind (themselves divided into the three sorts studied in 4.2), are called today subharmonics. Their study is the object of chapters XXVIII, XXX and XXXI. Poincaré describes them as follows in the analysis of his works:

Nous avons encore les solutions périodiques du deuxième genre; si l'on fait varier de manière continue un des paramètres dont dépend le problème, par exemple l'une des masses, on voit une solution périodique du premier genre se déformer d'une façon continue, sa période restant égale à $T$. A un certain

\footnotetext{
${ }^{139}$ In the same way, ergodic KAM tori of dimension 3 in the reduced phase space of the planar Three-Body Problem (the three frequencies being the two mean motions and the frequency of the difference of perihelia) give rise in the non reduced phase space to 4-dimensional invariant tori which could be resonant.

${ }^{140}$ The thesis of Anne Robadey Ro contains a beautiful study of the notion of "general case" in Poincaré's works on dynamical systems.

${ }^{141}$ Weierstrass has written that he had taught this theorem repeatedly in his courses since 1860 but he published it in 1886. Poincaré defended his thesis in 1879.

${ }^{142}$ Therefore, a periodic solution can disappear only after merging with another periodic solution. In other terms, the periodic solutions disappear by couples as do the real roots of algebraic equations.
} 
moment, cette solution se dédouble pour ainsi dire, ou plutôt se détriple, je veux dire qu'à un certain moment on a trois solutions périodiques très peu différentes; l'une d'elles a encore pour période $T$, les deux autres ont pour période un multiple de $T$. Ce sont les solutions périodiques du deuxième genre (Analyse des travaux) 143

In the case of a two degrees of freedom system, taking a surface of section transverse to the periodic solution of period $T$ under study reduces the problem to the existence of periodic points of an area preserving map in the neighborhood of an elliptic fixed point. In Chapter XXX, the study of these solutions is clearly presented as a companion to the study of asymptotic solutions, in today's vocabulary, it is the study of the dynamics attached to an elliptic periodic solution while the asymptotic solutions were attached to a hyperbolic one (see Figure 19, in a surface of section):
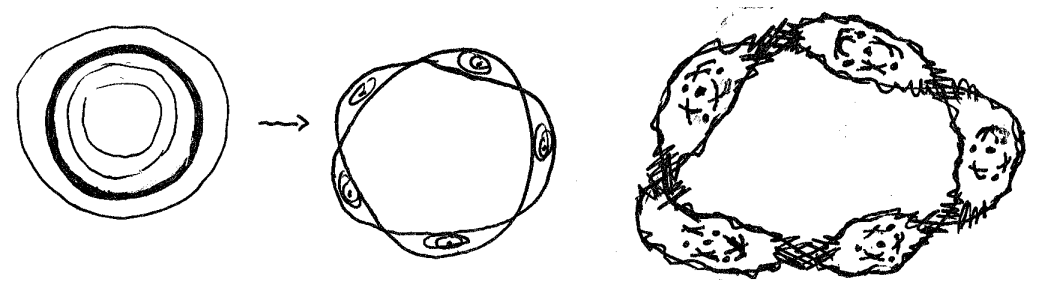

Figure 19: $3 T$-subharmonics around a periodic solution of the second sort.

Dans l'étude des solutions asymptotiques, nous avons supposé que les $\alpha_{k}$ étaient réels et nous avons annulé une des constantes $A$ sur deux.

Pour appliquer ce même résultat à l'étude des solutions périodiques du second genre, nous supposerons au contraire que les exposants $\alpha_{k}$ sont purement imaginaires. ${ }^{144}$

Introducing a parameter $\mu$ which controls the exponents of the periodic solution (in the surface of section, a fixed point wich one can suppose to be always at the origin), he shows that $k T$-subharmonics can bifurcate from the $T$-periodic solution, only in case one exponent of this solution is a multiple of $\frac{2 i \pi}{k T}$ (This happens for $\mu=\mu_{0}$ in Figure 20, drawn in the product of the surface of section and the parameter line).

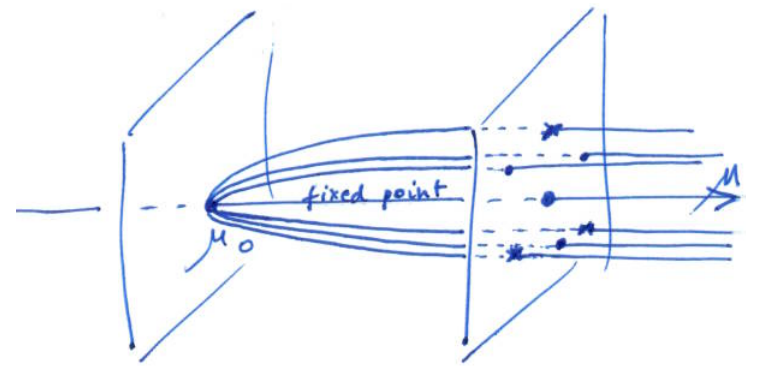

Figure 20: The birth of a pair of subharmonics of period $3 T$.

Moreover, he proves that

\footnotetext{
${ }^{143}$ We have still the periodic solutions of the second kind; if we vary continuously one of the parameters of which depends the problem, for example one of the masses, we see a periodic solution of the first kind deform in a continuous way, the period remaining equal to $T$. At some point, this solution splits into two, or rather three solutions, I mean that at some point we have three periodic solutions which differ only slightly; one of them still has period $T$, the period of the other two is a multiple of $T$. Those are the periodic solutions of the second kind (Analysis of the works).

${ }^{144}$ In the study of asymptotic solutions, we have supposed that the $\alpha_{k}$ were real and we have set half of the constants $A$ equal to zero. In order to apply this result to the study of the second kind periodic solutions, we shall suppose on the contrary that the exponents $\alpha_{k}$ are purely imaginary.
} 
il disparaît toujours autant de solutions stables que de solutions instables 145

and, on the other hand, that

Donc si, pour une certaine valeur de $\mu$, une solution périodique perd la stabilité ou l'acquiert (et cela de telle façon que l'exposant $\alpha$ soit nul) c'est qu'elle se sera confondue avec une autre solution périodique, avec laquelle elle aura échangé sa stabilité ${ }^{146}$

The very existence of the subharmonic solutions is reduced to the existence of critical points of a generating function: as the flow is symplectic, if we fix $T$ and call respectively $\left(\xi_{i}, \eta_{i}\right)$ and $\left(X_{i}, Y_{i}\right)$ the values at $\mathrm{t}=0$ and $t=T$ of $\left(x_{i}(t), y_{i}(t)\right)$,

$$
d S=\sum\left(\left(X_{i}-\xi_{i}\right) d\left(Y_{i}+\eta_{i}\right)-\left(Y_{i}-\eta_{i}\right) d\left(X_{i}+\xi_{i}\right)\right.
$$

is an exact differential. Indeed, being somewhat anachronic, we write

$$
d^{2} S=2\left(d X_{i} \wedge d Y_{i}-d \xi_{i} \wedge d \eta_{i}\right)=0 .
$$

One concludes if $S$ may be considered as a function of the $X_{i}+\xi_{i}$ and $Y_{i}+\eta_{i}$. After a chapter (XXIX, see 13.1) dedicated to the principle of least action (see section 13), Poincaré comes back to the subharmonics:

Je ne puis passer sous silence les rapports entre la théorie des solutions du deuxième genre et le principe de moindre action; et c'est même à cause de ces rapports que j'ai écrit le chapitre XXIX ${ }^{147}$ (see 13.1).

He analyses the way the subharmonics wrap around the $T$-periodic solution and identifies subharmonics with extrema of the action. The rotation number is not far.

But let us come back to the (Planar Circular Restricted) Three-Body Problem and more precisely to section 381 where Poincaré analyses a paper published in 1897 by George Darwin ${ }^{148}$ in the Acta Mathematica which follows numerically a family of periodic solutions of a Restricted Problem (the Sun, an enormous Jupiter and a small body): as he had done with the continuation of Hill's orbit (see 4.2), but arguing on the necessity of an exchange of stabilities, and pushing at the limit the examination of the different possibilities, he concludes

Donc, je conclus que les satellites $A$ instables ne sont pas la continuation analytique des satellites $A$ stables. Mais alors que sont devenus les satellites $A$ stables ?

Sur ce point, je ne puis faire que des hypothèses et, pour pouvoir faire autre chose, il faudrait reprendre les quadratures mécaniques de M. Darwin. Mais si l'on examine l'allure des courbes, il semble qu'à un certain moment l'orbite du satellite $A$ a dû passer par Jupiter et qu'ensuite il est devenu ce que M. Darwin appelle un satellite oscillant. ${ }^{149}$

This is of course a global problem for which there is a lack of tools, but not completely, as the next section shows.

\footnotetext{
${ }^{145}$ It always disappears as many stable solutions than unstable solutions.

${ }^{146}$ Therefore, if for some value of $\mu$, a periodic solution looses or acquires stability (and this in such a way that the exponent $\alpha$ be equal to zero) it is because it will have merged with another periodic solution with which it will have exchanged stability.

${ }^{147}$ I cannot omit to mention the relations between the theory of second kind solutions and the principle of least action; it is even because of these relations that I wrote chapter XXIX.

${ }^{148} \mathrm{~A}$ well-known astronomer, the son of sir Charles Darwin.

${ }^{149}$ Hence I conclude that the unstable satellites $A$ are not the continuation of stable satellites $A$. But then what
} 


\subsection{The last geometric theorem and twist diffeomorphisms of the annulus}

J'ai démontré il y a longtemps déjà, l'existence des solutions périodiques du problème des trois corps ; le résultat laissait cependant encore à désirer ; car si l'existence de chaque sorte de solution était établie pour les petites valeurs des masses, on ne voyait pas ce qui devait arriver pour des valeurs plus grandes, quelles étaient celles de ces solutions qui subsistaient et dans quel ordre elles disparaissaient 150

Poincaré published in 1912, the year of his death, an incomplete proof of what is called today the Poincaré-Birkhoff fixed point theorem [P15]. In a both lucid and moving introduction, he explains that he struggled during two years but could not reach a complete proof. Nevertheless, having proved the theorem in all the special cases he had examined and due to its paramount importance, he decided to submit the problem to geometers.

In 1905, he had already addressed more globally the question of periodic solutions on a problem similar to the Restricted Three-Body Problem but "stripped from the secondary difficulties", namely the geodesics on a convex surface [P14, Ro]. Comparing this question with the one completely understood by J. Hadamard Ha] in the case of negative curvature ${ }^{151}$ he writes:

J'ai donc abordé l'étude des lignes géodésiques des surfaces convexes ; malheureusement, le problème est beaucoup plus difficile que celui qui a été résolu par M. Hadamard. J'ai donc dû me borner à quelques résultats partiels, relatifs surtout aux géodésiques fermées, qui jouent ici le rôle des solutions périodiques du problème des trois corps ${ }^{152}$

The fixed point theorem will be proved by Birkhoff during the year following Poincaré's death [Bi1] (for a radically different proof, see [BH]); it asserts the existence of at least two fixed points of an area preserving twist diffeomorphism ${ }^{153}$ of the annulus $S^{1} \times[0,1]$. The application to the Restricted Three-Body Problem with a large Jacobi constant, comes from the fact that, the return map in the Birkhoff annulus of section ${ }^{154}$ is a monotone twist map ${ }^{155}$ which allows application of the theorem to its iterates in order to get periodic orbits. But in fact, the full force of the theorem is not needed in this case or in the companion case of an area preserving diffeomorphism of the plane in the neighborhood of a generic elliptic fixed point. The main features which are understood are represented on Figure 21 (see [Ze, C9, Le]):

\footnotetext{
happened with the stable satellites $A$ ? About this, I can only make hypotheses and, in order to get something else one shoud continue the mechanical quadratures of M. Darwin. But, if one examines the shape of the curves, it seems that at some moment the orbit of the satellite must have passed near Jupiter and that after that he became what M. Darwin calls an oscillating satellite.

${ }^{150} \mathrm{I}$ showed long ago, the existence of periodic solutions of the Three-Body Problem; nevertheless, the result was still unsatisfactory; for, if the existence of each type of solution was established for small values of the masses, one did not see what would happen for larger values, which ones of these solutions remained and in what order they disappeared.

${ }^{151}$ in which case, the periodic geodesics are indeed dense in the phase space and moreover a full description of the flow with symbolic dynamics is available.

${ }^{152} \mathrm{So}$, I approached the study of geodesic lines of convex surfaces; unfortunately the problem is much more difficult than the one solved by M. Hadamard. Hence I had to content myself with some partial results, mainly concerning closed geodesics, which play here the role of periodic solutions of the Three-Body Problem.

${ }^{153}$ i.e., a diffeomorphism of $S^{1} \times[0,1]$ which admits a lift to a diffeomorphism of $\mathbb{R} \times[0,1]$ which is increasing on one boundary and decreasing on the other.

${ }^{154}$ See Figure 15 and $\mathrm{Co}$.

155 i.e., there exists an identification of the annulus of section to $S^{1} \times[0,1]$ such that the image by the return map of any "vertical" segment $\theta_{0} \times[0,1]$ is a graph over a part of the circle (see [C9, Le] ).
} 


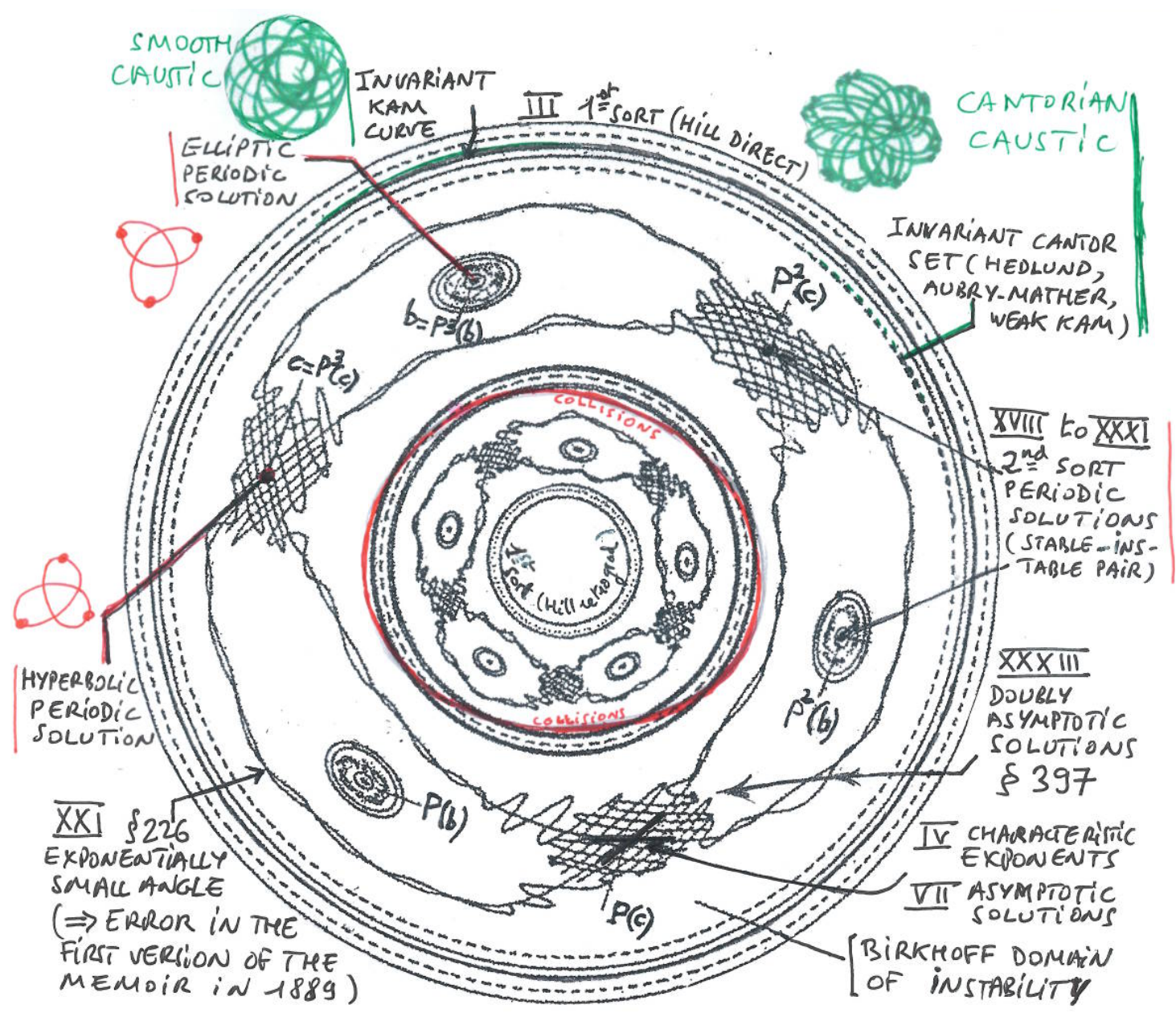

Figure 21: The Planar Circular Restricted Three-Body Problem in the case of a high Jacobi constant (Birkhoff global annulus of section).

1) The KAM invariant curve $\$^{156}$ on which the return map is analytically conjugate to a rotation of angle $2 \alpha \pi$ with $\alpha$ an irrational satisfying a Diophantine condition. They imply STABILITY! Generically, the union of these invariant curves is a set whose transversal struture is Cantor-like. The annuli in between two invariant curves (and not containing in their interior any invariant curve homotopic to the boundary) are called Birkhoff zones of instability. The dynamics in such a zone is quite complicated and certainly not completely understood. The main features are

2) "well-ordered" periodic orbits ${ }^{157}$ obtained by minimization of a generating function; the homoclinic and heteroclinic tangles associated with the invariant manifolds of the hyperbolic periodic orbits (see [Ze]);

3) the Aubry-Mather invariant Cantor sets and the invariant manifolds attached to them, part of which make the weak KAM solutions. These sets, whose existence was initially obtained by S. Aubry and J. Mather, in each case by minimizing the action functional, were shown by A. Katok to be limits of periodic orbits, thanks to the Birkhoff a priori Lipschitz estimates resulting from the monotone twist condition;

\footnotetext{
156 which, in the case of a return map, are the trace of the KAM tori on a surface of section.

157 i.e., circularly ordered as a periodic rotation; thanks to the monotone twist condition, one gets periodic orbits much better controlled than the ones given by the Poincaré-Birkhoff fixed point theorem applied to iterates of the return map.
} 
4) diffusing orbits found by Birkhoff and refined by Mather using a variational apporach, which go from one component of the boundary to the other one and may be imposed to pass through the Aubry-Mather sets in a prescribed way ${ }^{158}$

5) and the same phenomena repeated around the subharmonics, etc etc. But we still do not know whether periodic points are dense or if hyperbolic behaviour (that is non-zero Lyapunov exponents) exist on a set of positive Lebesgue measure.

The Poincaré-Birkhoff fixed point theorem, which makes more global part of the above results, has played a very important role in the development of symplectic topology, in particular through the proofs of various instances of the Arnold conjecture relating the number of fixed points of Hamiltonian diffeomorphisms of compact manifolds and Morse theory. The breakthrough was the paper [CZ by Charles Conley and Eddy Zehnder, followed by a reduction to the finite dimension of their proof by Marc Chaperon Chap, well in the spirit of Poincaré's use of generating functions. Describing this would lead us too far astray, hence I refer to $\mathrm{Au} 2$ for this part of the story and the references.

\subsection{Computer experiments and the geometrization of phase space}

In the twentieth century, an extensive search for families of periodic solutions in the Restricted Three-Body Problem was accomplished, first by mechanical quadratures at the Copenhagen Observatory (Stromgren), later using computers by Hénon at the Nice Observatory, Broucke, and others. The books by Szebehely [Sz], Hénon $[\mathrm{He}$, Bruno $[\mathrm{Br}]$ describe the Planar Circular Restricted Three-Body Problem both theoretically and numerically.

Particularly interesting for mission design are the Halo orbits in the spatial Restricted Problem, which bifurcate from a planar Lyapunov family originating from a collinear relative equilibrium. Other much studied special cases are the collinear problem with the remarkable periodic (regularized) solution discovered by Schubart (see an animation in [C4]) and the isosceles problem, where one body moves on a line, while the two others, with the same mass, move symmetrically on the orthogonal line (resp. plane in the spatial case).

Today, the power of computers allows us to get a pretty good understanding of autonomous 2 degrees of freedom systems like the Restricted Problem or its simplified version called the Hill problem. For example, an extensive study of the phase space of the related Hill's problem was completed by Simó and Stuchi [ST]; computer assisted proofs of the existence of KAM tori (and hence of stability) for realistic (Sun-Jupiter-Ceres or Sun-Jupiter-Victoria), but truncated to a trigonometric polynomial, Restricted Three-Body Problems, were given by Alessandra Celletti and Luigi Chierchia in [CC1, CC2].

Finally, results of C. Simó and his colloborators show the important role played by invariant manifolds or, more generally, of center manifolds of (families of) periodic solutions, in determining the domains of practical stability of celestial bodies. See for instance [Si, SST]. In some sense, this shows that Poincaré was not completely wrong when trying to prove stability from the behavior of these invariant manifolds.

\footnotetext{
${ }^{158}$ The variational techniques having led to these results are currently used in order to prove the generic existence of diffusion in 2.5 or 3 degrees of freedom Hamiltonian systems.
} 


\section{A great principle of physics and some collisions}

In The value of Science [P13], Poincaré puts the Principle of least action on the same footing as the great conservation principles (energy, mass, action-reaction), the principle of degradation of energy and the relativity principle. Due to its global character it may appear at first sight closer to theology than to physics and indeed, in chapter VIII of Science and Hypothesis Poincaré wonders:

L'énoncé même du principe de moindre action a quelque chose de choquant pour l'esprit. Pour se rendre d'un point à un autre, une molécule matérielle soustraite à l'action de toute force mais assujettie à se mouvoir sur une surface, prendra la ligne géodésique, c'est-à-dire le chemin le plus court.

Cette molécule semble connaître le point où on veut la mener, prévoir le temps qu'elle mettra à l'atteindre en suivant tel et tel chemin, et choisir ensuite le chemin le plus convenable. L'énoncé nous la présente pour ainsi dire comme un être animé et libre. Il est clair qu'il vaudrait mieux le remplacer par un énoncé moins choquant, et où, comme diraient les philosophes, les causes finales ne sembleraient pas se substituer aux causes efficientes 159

In [Fey] (volume I, Chap. 26, par. 5: A more precise statement of Fermat's principle), Richard Feynman asks the same question, but with this time the answer given by quantum electrodynamics, that is by the principle of stationary phase:

Instead of saying it is a causal thing, that when we do one thing, something else happens, and so on, it says this: we set up the situation, and light decides which is the shortest time, or the extreme one, and chooses that path. But what does it do, how does it find out? Does it smell the nearby paths, and check them against each other? The answer is, yes, it does in a way.

\subsection{Least action and instability}

Chapter XXIX of The New Methods opens with a crash course in elementary Calculus of variations applied to the action integrals $\int \sum_{i} p_{i} d q_{i}$ (the Maupertuisian action) when fixing the energy and $\int\left(\sum_{i} p_{i} d q_{i}-H d t\right)$ (the Hamiltonian action) when fixing the time interval 160 Of course, in the case of the Three-Body Problem, $\sum p_{i} \frac{d q_{i}}{d t}-H$ is simply the Lagrangian in the disguise provided by the Legendre transformation and I suppose that, if there is a single notion which need not be explained to physicists, it must be the Lagrangian action $\int L d t$.

Then, developing the note [P10] which was based on a simple geometric reasoning, he proves that a periodic solution which locally minimizes the action must be dynamically unstable:

\footnotetext{
${ }^{159}$ The very statement of the principle of least action has something shocking to the mind. To go from one point to another one, a material molecule, taken away from the action of any force, but constrained to move on a surface, will follow the geodesic line, i.e., the shortest path. It seems that this molecule knows the point where one wants it to go, that it anticipates the time needed to reach it along such or such path, and then chooses the most convenient path. In a sense, the statement presents this molecule as a free animated being. It is clear that it would be better to replace it by a less shocking statement where, as philosophers would say, the final causes would not appear to replace the efficient ones.

${ }^{160}$ See Remark 1 at the end of section 8.1
} 
En résumé, pour qu'une courbe fermée corresponde à une action moindre que toutes les courbes fermées infiniment voisines, il faut et il suffit que cette courbe fermée corresponde à une solution périodique instable de la première catégorie 161

In particular, a "generic" closed geodesic on a surface locally minimizes the length among nearby closed loops if and only if it is hyperbolic (and hence unstable), i.e., if its non-trivial exponents are real. For example, on a torus of revolution around the $z$ axis, the intersections of the torus with the horizontal plane of symmetry are respectively unstable for the short one, along which the curvature is negative, and stable for the long one, along which the curvature is positive: along the first one there are no conjugate points while they exist on the second one.

Such assertions hold only for mechanical systems with two degrees of freedom. Indeed, examples studied by Marie-Claude Arnaud in [Ar] show that in higher dimensions, a locally action minimizing periodic solution may possess only two directions of instability transverse to the flow in its energy level.

\subsection{Minimizing the action: the note of $\mathbf{1 8 9 6}$}

In 1896, Poincaré publishes the short note [P5], titled On the periodic solutions and the least action principle. The note of 1897 which was quoted in section 13.1 will have almost the same title, but this one is much more ambitious, even if not directly applicable to the Newtonian force. Indeed, in the analysis of his works, Poincaré writes:

Je suis revenu sur ces solutions périodiques et je les ai étudiées en détail. Les procédés dont je me suis servi pour démontrer leur existence sont très simples et se ramènent au calcul des limites.

Mais on peut arriver à cette démonstration par une voie toute différente, qu'il pourra être souvent utile d'adopter, mais dont je n'ai pas encore tiré tout le parti possible. Supposons par exemple que l'on recherche les géodésiques d'une surface indéfinie présentant la même forme générale qu'un hyperboloïde à une nappe. On sera certain alors qu'il doit y avoir une géodésique fermée (correspondant à une solution périodique) parce que, parmi toutes les courbes fermées que l'on peut tracer sur la surface et qui en font le tour, il doit y en avoir une qui est plus courte que toutes les autres.

Les mêmes principes sont susceptibles d'être appliqués à divers problèmes de Mécanique, grâce au principe de moindre action que l'on peut employer soit sous la forme que lui a donnée Hamilton, soit sous celle que lui a donnée Maupertuis. Je n'ai fait qu'esquisser cette méthode dont il y a sans doute encore beaucoup à tirer 162

\footnotetext{
${ }^{161}$ In short, for a closed curve to correspond to a lesser action than all closed curves which are infinitely close, it is necessary and sufficient that the closed curve corresponds to an unstable periodic solution of the first category.

${ }^{162}$ I came back to these periodic solutions and I studied them in detail. The methods I used to prove their existence are very simple and can be reduced to the calculus of limits. But one can arrive to this proof by a completely different path, which it will often be useful to follow, but which I did not fully exploit. Suppose, for instance, that one is looking for the geodesics of an indefinite surface whose general shape is the one of a one-sheeted hyperboloid. One can be sure that there exists a closed geodesic (corresponding to a periodic solution) because, among all the closed curves going around the surface which one can draw, one must be shorter than the others. Thanks to the Principle of Least Action, used in the form given by Hamilton or in the form given by Maupertuis, the same principles can
} 
Referring to [C2 for more details and references, I quickly describe what Poincaré does: he is looking for relative periodic solutions of the planar Three-Body Problem, i.e., solutions which after the period $T$ are such that the triangle formed by the three bodies is the same up to a rigid rotation around the center of mass (which one can suppose to be fixed). Given a relative loop of configurations of the three bodies in the plane (i.e., a family parametrized by the interval $[0, T]$ of triangles changing their shape and position but keeping their center of mass fixed and coming back to the same triangle up to a rotation $\theta \bmod 2 \pi)$, he calls respectively $\theta, \theta+2 k \pi, \theta+$ $2 l \pi, k, l \in \mathbb{Z}$ the total (i.e., not $\bmod 2 \pi$ ) angles described by the three sides of the triangle during one period. Then, looking for the simplestpossible solutions in this class, he minimizes the Lagrangian action $\int_{0}^{T}\left[\frac{1}{2} \sum_{i} m_{i} \| \frac{d \vec{r}_{i}}{d t}||^{2}+\sum_{i<j} \frac{m_{i} m_{j}}{\left\|\vec{r}_{i}-\vec{r}_{j}\right\|}\right] d t$ of the Three-Body Problem in the subspace of those relative loops for which the integers $k$ and $l$ are fixed. In fact this was fixing the homology class of the loop of relative configurations. It is interesting to recall that the year before, Poincaré had defined the fundamental group. Choosing $k$ and $l$ different from zero implies coercivity i.e., forbids a minimizer to be "at infinity". Actually, it is only around 1930 that the existence of an absolutely continuous minimizer was proved by Tonelli provided coercivity holds, its regularity following from Weierstrass theory; but Poincaré does not even mention the question. He does not mention either the major problem, the possibility for a minimizer of having collisions and hence not being a true solution: it is an important result of Sundman around 1913 that, in a motion of $n$ point masses under Newtonian attraction, if two or more bodies collide at a certain instant $t_{0}$, their mutual distances and mutual velocities satisfy the same estimates as do colliding bodies in the Two-Body Problem

$$
\left\|\vec{r}_{i}-\vec{r}_{j}\right\|=O\left(\left|t-t_{0}\right|^{\frac{2}{3}}\right) \quad \text { and } \quad\left\|\frac{d \vec{r}_{i}}{d t}-\frac{d \vec{r}_{j}}{d t}\right\|=O\left(\left|t-t_{0}\right|^{-\frac{1}{3}}\right)
$$

This relative weakness of the Newtonian attraction implies that the action stays finite even if a collision occurs and leaves the possibility that minimizers have collisions. Well aware of the problem, Poincaré avoids it by supposing from the start a strong force attraction, that is a force function $U$ which is a sum of terms of the form $\frac{\mathrm{kmm}^{\prime}}{\mathrm{r}^{n}}$, with $n$ at least equal to 2. He may then argue later on:

Je dis maintenant que, si $a$ et $c$ se rencontrent, l'action est infinie.

En effet, l'action sera du même ordre de grandeur que $\int 2 U d t$, ou que $\int 2 \sqrt{U} d r$, ou que $2 k m m^{\prime} \int \frac{d r}{r^{\frac{n}{2}}}$, c'est-à-dire infinie si $n \geq 2$. Or, on a $n=2$ si, comme nous le supposons, l'attraction s'exerce en raison inverse du cube des distances 163

This is cheating of course (and also forgetting the possibility of triple collisions), but at the same time very typical of Poincaré's bold exploration of a new domain: going further at a quick pace, while being well aware of the questions to which he should come back ... if time permits.

be applied to various problems of Mechanics. I have only sketched this method from which there is still probably much to get.

${ }^{163}$ I say now that, if $a$ and $c$ meet, the action is infinite. Indeed, the action will be of the same order of magnitude as $\int 2 U d t$, or as $\int 2 \sqrt{U} d r$, or as $2 k m m^{\prime} \int \frac{d r}{r^{\frac{n}{2}}}$, that is infinite if $n \geq 2$. But, one has $n=2$ if, as we suppose, the attraction is proportional to the inverse cube of the distances. 
As we know, there was no time for Poincaré to come back to this question and this note was forgotten during a century. For instance, it is not mentioned by William B. Gordon who, in 1977, shows that minimizers of the action $\int_{0}^{T}\left(\left.\frac{1}{2}\left\|\frac{d \vec{r}}{d t}\right\|\right|^{2}+\frac{k}{\|\vec{r}\|}\right) d t$ of the Kepler Problem in the plane (Newtonian attraction by a fixed center at the origin of $\mathbb{R}^{2}$ ) among loops of index $k$ around the origin, are the Kepler ellipses of period $T$ if $k= \pm 1$ while if $|k| \geq 2$, they lie on the boundary of the chosen set of loops, being the ejection-collision solutions of period $T$, whose action is strictly smaller than the one of the elliptic solutions of period $T / k$ described $k$ times!

Indeed, constraining the homology of the loop of configurations (be it relative or absolute) most of the time leads to minimizers with collisions (there are exceptions found by A. Venturelli, K.C. Chen,...). But, if one replaces the homology constraint by a symmetry constraint, the method becomes remarkably efficient, leading in particular to new classes of periodic or quasi-periodic solutions, the choreographies and the Hip-Hops (see the references in C2, Mont1, Mont2,). The important fact behind this success is Marchal's theorem [Ma2, C8, C5] which asserts that, for the $N$-body problem in the plane or in space, minimizers of the action in given time between two fixed configurations have no collision.

\subsection{Solutions of the second species}

In spite of the fact that, in Poincaré's sketch of the existence of these solutions, there is no appeal to the principle of least action, a recent proof by Bolotin and Negrini $[\mathrm{BN}]$ is based on it, hence the choice to put this section at the end of the one on the principle of least action. Being interested mainly in planetary systems of the type of the solar system, Poincare was not much concerned with collisions ${ }^{164}$ In the note [P5] that was discussed in the previous section, he even surreptitiously changed the law of attraction in order to avoid them. In The New Methods, there is only one chapter, which indeed displays a quite remarkable intuition, where he copes with solutions approaching collisions, in fact only two-body collisions. The idea is simple and beautiful: in the limit of zero masses, the planets follow Keplerian orbits till they have a close encounter (i.e., in the limit, a collision) and then shift to another pair of Keplerian ellipses:

A full symbolic dynamics of such almost collision orbits has been constructed by Bolotin: it implies the existence of solutions with an erratic diffusion of the angular momentum and a much slower one of the Jacobi constant. Existence in the full ThreeBody Problem is proved in $[\mathrm{BN}]$ where one can find references to the previous works in the Restricted (Circular or Elliptic) Problem.

If one replaces almost double collision by almost triple collision, the situation becomes tremendously richer. The way to analyse it is to compactify by adding the so-called McGehee collision manifold (see [McG, C7]). A description of some of the complexity it implies in the phase portrait of the planar Three-Body Problem can be found in the beautiful survey paper by Rick Moeckel [Moe].

\footnotetext{
${ }^{164}$ Nevertheless, as we saw in section 8.1 he understood the scaling invariance of the equations, fundamental in the studies of collisions.
} 


\section{Resonances 3) diffusion}

\subsection{Arnold diffusion}

Kolmogorov's result implies a very strong stability result in the (planar circular) Restricted Problem. Indeed invariant curves are barriers, playing the role that Poincaré had wanted to assign to the separatrices of the hyperbolic periodic orbits in the first version of his Memoir of 1889. Indeed, as noticed by Arnold in the russian version of Poincaré's works, in the case of 2 degrees of freedom, the existence of KAM invariant tori implies the existence of non trivial $C^{\infty}$ integrals.

As is well-known today, the situation is much more complex in the non restricted case where, the number of degrees of freedom being strictly greater than 2, Lagrangian invariant tori do not separate an energy manifold. This leaves room for a very slow diffusion of the trajectories not belonging to the invariant tori: remaining a long time around the KAM tori which are quite sticky, they could a priori escape very far. The first (highly non generic) example of this phenomenon was given by Arnold in 1964 [A4.

Lower bounds on diffusion time for close to integrable Hamiltonian systems were first given by Nekhoroshev [Ne. An example of application to the Three-Body Problem is [Nie. Proving that this phenomenon occurs in a specific (and not ad hoc) problem or even generically is very hard; recent examples for the Three-Body Problem are GK] and [FGKR] for the Planar Elliptic Restricted Three-Body Problem. For the long-term evolution of the full solar system, see [Las1].

\subsection{The oldest open question in dynamical systems}

In the 1998 International congress of mathematicians, which took place in Berlin, Michael Herman [Her] ended his beautiful survey of open problems with the following question:

For the $n$-body problem in space, we will suppose $n \geq 3$.

- The center of mass is fixed at 0 .

- On the energy surface we $C^{\infty}$-reparametrize the flow by a $C^{\infty}$ function $\varphi_{e}$ (after reduction of the center of mass) such that the flow is complete: we replace $H$ by $\varphi_{e}(H-e)=H_{e}$ so that the new flow takes an infinite time to go to collisions ( $\varphi_{e}$ is a $C^{\infty}$ function outside collisions).

Following G.D. Birkhoff [Bi] (who only considers the case $n=3$ and the angular momentum $\neq 0$ ) (see also A.N.Kolmogorov $[\mathrm{K}]$ ), we ask:

Question. Is for every e the non-wandering se ${ }^{165}$ of the Hamiltonian flow of $H_{e}$ on $H_{e}^{-1}(0)$ nowhere dense in $H_{e}^{-1}(0)$ ?

In particular, this would imply that the bounded orbits are nowhere dense and no topological stability occurs.

It follows from the identity of Jacobi-Lagrange that when $e \geq 0$, every point such that its orbit is defined for all times, is wandering.

The only thing known is that, even when $e<0$, wandering sets do exist (Birkhoff and Chazy, see Alexeyev [Ale]).

\footnotetext{
${ }^{165} \mathrm{~A}$ point is wandering if some neighborhood of it, when propagated by the flow of the differential equation, never comes back to intersect itself
} 
The fact that the bounded orbits have positive Lebesgue-measure when the masses belong to a non empty open set, is a remarkable result announced by V.I. Arnold [A1] (Arnold gives only a proof for [the] planar 3-body problem and if the author is not mistaken, Arnold's claim is correct).

In some respect Arnold's claim proves that Lagrange and Laplace, against Newton, are correct in the sense of measure theory and that, in the sense of topology, the above question, in some respect, could show Newton to be correct 166

In order to know the answer of astronomers to this question, the best is to read the report by Jacques Laskar in this seminar [Las1]. Indeed, as he told me in front of a chinese tea, for realistic masses of the planets, instability seems likely, even in the sense of measure!

One may contrast this with the words of Poincaré at the beginning of [P8].

Les personnes qui s’intéressent aux progrès de la mécanique céleste, mais qui ne peuvent les suivre que de loin, doivent éprouver quelque étonnement en voyant combien de fois on a démontré la stabilité du système solaire.

Lagrange l'a établie d'abord, Poisson l'a démontrée de nouveau, d'autres démonstrations sont venues depuis, d'autres viendront encore. Les démonstrations anciennes étaient-elles insuffisantes, ou sont-ce les nouvelles qui sont superflues?

L'étonnement de ces personnes redoublerait sans doute, si on leur disait qu'un jour peut-être un mathématicien fera voir, par un raisonnement rigoureux, que le système planétaire est instable.

Cela pourra arriver cependant; il n'y aura là rien de contradictoire, et cependant les démonstrations anciennes conserveront leur valeur. ${ }^{167}$

In fact, in his conclusion, greatly overestimating the importance of dissipative forces 168 in particular of the tides, he concludes that their action makes illusory, at the astronomical level, any further progress in the mathematical analysis of the abstract problem of stability.

\footnotetext{
${ }^{166}$ It is in the Opticks $[\mathrm{N}]$ that Newton says that God must from time to time set the solar system back into order:

For while Comets move in very excentrick Orbs in all manner of Positions, blind Fate could never make all the Planets move one and the same way in Orbs concentrick, some inconsidereable Irregularities excepted, which may have risen from the mutual Actions of Comets and Planets upon one another, and which will be apt to increase, till this System wants a Reformation.

while, as we saw in 3.3 Lagrange and Laplace show stability at first order by studying the secular system linearized at circular and coplanar motions.

${ }^{167}$ The persons interested in the advances of celestial mechanics but who can only follow them from far, must be somewhat astonished when seeing how many times the stability of the Solar System was proved. Lagrange first proved it, then Poisson, other proofs followed, other are still to come. Were the ancient proofs no sufficient, or is it that the new ones are superfluous? The astonishment of these persons would certainly increase, if they were told that it may happen that some day a mathematician will show, by a rigourous reasoning, that the planetary system is unstable. Nevertheless, this might well happen; and yet there will be no contradiction, and the ancient proofs will keep their value.

${ }^{168}$ Indeed, according to Las2, in the solar system (but not in some extrasolar systems) the conservative diffusion of trajectories greatly dominates the dissipative forces.
} 


\section{Surprises of a eulogy}

On December 15th 1913, Gaston Darboux pronounces at the Paris Academy of Science the eulogy of Henri Poincaré [D]. I do not resist the pleasure of reproducing the beginning of his section VII:

Poincaré ne pouvait pas ne pas avoir conscience de la haute valeur de ses écrits; d'autres auraient réclamé des récompenses, lui ne demandait rien. Nous le regardions tous comme le plus fort d'entre nous. Il n'a jamais cherché à nous devancer. Nul ne pouvait prévoir les vides nombreux que la mort allait faire dans la section de Géométrie. Pour le faire arriver plus vite, pour lui ménager une place dans la section d'Astronomie, on lui signalait les applications que les théories par lui découvertes pouvaient avoir en Mécanique céleste. Il suivait docilement ces indications, s'occupait du problème des trois corps, des figures des corps célestes, et trouvait tout naturel de laisser passer devant lui tous les anciens ${ }^{169}$

\section{A seminar}

From 1988 to 1990, Jacques Laskar and the author held in the Bureau des Longitudes (of which Poincaré had been a member since 1893 and three times president, a threeyear-long seminar dedicated to reading the three volumes of The New Methods of Celestial Mechanics. Being attended by astronomers and mathematicians, the seminar was at the origin of the creation of ASD (Astronomie et Systèmes Dynamiques), a group in which we try to perpetuate the collaboration between astronomers and mathematicians. We knew that Poincaré's researches on differential equations, and in particular on the Three-Body Problem, had created whole chapters of today's mathematics: dynamical systems, differential forms, ergodic theory, topology, ..., but we have discovered in the course of these three years the visionary precision with which these three volumes exposed ideas which we had thought recent. The items [S1, S2, S3] were published at that time.

\section{Thanks}

to Alain Albouy, Jacques Féjoz, Jacques Laskar, Philippe Robutel, and all members of the group ASD (Astronomy and Dynamical Systems) for years of illuminating discussions on these matters and others. Thanks to Daniel Bennequin, Alexey Borisov, Thierry Combot, Sylvio Ferraz-Mello, Yanning Fu, Hugo Jiménez-Pérez, Yvette Kosmann-Schwarzbach, Christian Marchal, Jessica Massetti, Rick Moeckel, Carles Simó, Jacques-Arthur Weil, Lei Zhao, for precisions, documents, references, corrections. Special thanks to Jacques Féjoz, Bertram E. Schwarzbach, Tadashi Tokieda, Scott Walter, for everything which sounds like good English.

\footnotetext{
${ }^{169}$ Poincaré could not be unaware of the high value of his writings; others would have claimed awards, he asked nothing. We all considered him as the strongest of us all. He never attempted overtaking us. No one could foresee the many gaps that death woud cause in the Geometry section. In order to make him arrive faster, to secure for him a seat in the Astronomy section, we pointed to him the applications that the theories he had discovered could have in Celestial Mechanics. He was obediently following these indications, handling the Three-Body Problem, the figures of celestial bodies, and finding it quite natural to let pass before him the elders.
} 


\section{Regret}

Celui de vivre à une époque où la belle langue de Poincaré est de moins en moins audible, comme en témoigne l'écriture en anglais de ce texte, demandée par les organisateurs du "séminaire Poincaré", que je remercie néanmoins pour leur souhait de rendre à Poincaré l'hommage des physiciens d'aujourd'hui (sorry, the tribute of contemporary physicists).

\section{Note on the references}

While writing this text, I have made free use of parts of my former papers on Poincaré's works on the $N$-body Problem: [S1, S2, C1, C2, C3]. The last three are available on my page of preprints:

http://www.imcce.fr/Equipes/ASD/person/chenciner/chenciner.html

Giving all the sources for the concepts involved was of course beyond the scope of this paper, hence I have often given priority to easier-to-read expositions and in particular to the ones I understand best, that is my own. Nice general descriptions respectively of the Two-body and Three-Body Problem are [Alb2, Ma1, as well as the classical reference [Wi] which has very interesting historical notices. Some of the figures are taken from the slides of a lecture given on The New Methods at Paris Observatory on July 9 of this year (hence the annotations in French). These slides are available at http://www.imcce.fr/poincare2012/ or at my address above under the heading "Quelques conférences". Finally, for those who are able to read French, I remind you that many of Poincaré's papers and letters are available, with many other documents, at the address http://www.univ-nancy2.fr/poincare/documents/bd1.html.

\section{References}

[S1] A. Chenciner, Intégration du Problème des deux corps par la méthode de Hamilton-Jacobi : les coordonnées action-angle de Delaunay,

J. Laskar Les variables de Poincaré et le développement de la fonction perturbatrice, note technique S026 du Bureau des Longitudes, 1989.

[S2] A. Chenciner, Séries de Lindstedt, note technique S028 du Bureau des Longitudes, 1990.

[S3] S. Ferraz-Mello, The Method of Delaunay; A. Jupp Chapter XIX Bohlin Method, note technique S036 du Bureau des Longitudes, 1992.

[AA] K. Abdullah et A. Albouy, On a strange resonance noticed by M. Herman, Regular and chaotic dynamics, V. 6, 4 (2001), 421-432.

[Alb1] A. Albouy, Histoire des équations de la mécanique analytique : repères chronologiques et difficultés, in "Siméon-Denis Poisson, les mathématiques au service de la science" (Y. Kosmann-Schwarzbach ed.), éditions de l'École polytechnique, to appear.

[Alb2] A. Albouy, Lectures on the Two-body Problem, Classical and Celestial Mechanics (The Recife lectures), H. Cabral and C. Diacu ed., Princeton University Press (2002). 
[AC] A. Albouy \& A. Chenciner, Le problème des $n$ corps et les distances mutuelles, Inventiones Mathematicæ, 1997, Volume 131, Number 1, Pages 151-184.

[Ale] V. M. Alexeyev, Sur l'allure finale du mouvement dans le problème des trois corps, ICM Nice 1970, 2, 893-907.

[d'Al] d'Alembert, Euvres complètes de Jean Le Rond D'Alembert : premiers textes de mécanique céleste 1747 - 1749, ed. by Michelle Chapront-Touzé, CNRS Ed.

[Ar] M. C. Arnaud, On the type of certain periodic orbits minimizing the Lagrangian action, Nonlinearity (1998) Vol. 11, 143-150.

[A1] V. I. Arnold, Petits dénominateurs et problème de la stabilité du mouvement en mécanique classique et céleste, Usp. Mat. Nauk 18 (1963), 91-192.

[A2] V. I. Arnold, Mathematical Methods of Classical Mechanics, Graduate Texts in Mathematics, Vol. 60, 2nd edition, Springer, 1989.

[A3] V. I. Arnold, Proof of a theorem of A. N. Kolmogorov on the invariance of quasi-periodic motions under small perturbations of the Hamiltonian, 1963.

[A4] V. I. Arnold, Instability of dynamical systems with several degrees of freedom, Dokl. Akad. Nauk SSSR 156, no 1, 9-12 (1964) (Russian); English translation: Sov. Math. Dokl. 5, 581-585 (1964).

[AKN] V. I. Arnold, V. V. Kozlov \& A. I. Neishtadt, Mathematical Aspects of Classical and Celestial Mechanics, 3rd ed., Dynamical Systems III, Encyclopædia of Mathematical Sciences, vol. 3, Springer 2006.

[Au] M. Audin, Intégrabilité et non-intégrabilité de systèmes hamiltoniens [d'après S. Ziglin, J. Morales-Ruiz, J.P. Ramis], séminaire Bourbaki 884, mars 2001 http://www. numdam.org/numdam-bin/feuilleter?id=SB_2000-2001__43_.

[Au2] M. Audin, Vladimir Igorevitch Arnold and the invention of symplectic topology, preprint 2012.

[BG] J. Barrow-Green, Poincaré and the Three-Body Problem AMS, History of Mathematics, vol. 11, (1997).

[Bi] G. D. Birkhoff, Dynamical Systems, Chapter IX, AMS, (1927).

[Bi1] G. D. Birkhoff, Proof of Poincaré's geometric theorem, Trans. Amer. Mat. Soc. Jauary 1913, Vol. 14, p. 14-22.

[BN] S. Bolotin and P. Negrini, Variational apporach to second species periodic solutions of Poincaré of the 3-body problem, Discrete and Continuous Dynamical Systems, Volume 33, Number 3, March 2013, pp. 1009-1032.

[Boh] K. Bohlin, Über eine neue Annäherungsmetode in der Störungsteorie, Bihang till K. Svenska Vet.-Akad. 14 Afd. I, No. 5, Norstedt, Stockholm, 1888; partial republication in 1889 in Astronomisches Nachrichten.

[Bo] J. L. Borges, Ficciones, Editorial Sur, Buenos Aires (1944).

[BH] B. Bramham and H. Hofer First steps towards a symplectic dynamics, Surveys in Differential Geometry Volume 17 (2012) Number 1 
[Br] A. D. Bruno, The restricted 3-Body Problem: Plane Periodic Orbits, de Gruyter (1994).

[Bru] H. Bruns, Über die Integrale des Vielkörper-problems, Acta Mathematica, volume 11 (1887).

[CMS] C. Carminati, S. Marmi and D. Sauzin, There is only one KAM curve, preprint 2011, http://hal .archives-ouvertes.fr/hal-00585973/.

[Ca] E. Cartan, Leçons sur les invariants intégraux, Hermann, Paris 1922.

[Cas] G. Casale, Morales-Ramis theorems via Malgrange pseudogroup, Ann. Inst. Fourier (Grenoble) 59 (2009), no 7, 25932610.

[CC1] A. Celletti and L. Chierchia, On the stability of realistic Three-Body Problems, Comm. Math. Phys. 186 (1997), nº 2, 413-449.

[CC2] A. Celletti and L. Chierchia, KAM stability and celestial mechanics, Mem. Amer. Math. Soc. 187 (2007), no 878.

[Chap], Une idée du type géodésiques brisées pour les systèmes hamiltoniens, C. R. Acad. Sci. Paris Sér. I Math. 298 (1984), nº 13, p. 293-296.

[Cha] C. L. Charlier, Die Mechanik des Himmels, de Gruyter 1927.

[C0] A. Chenciner, Systèmes dynamiques différentiables, Encyclopédie Universalis.

[C1] A. Chenciner, De la mécanique céleste à la théorie des systèmes dynamiques, aller et retour : Poincaré et la géométrisation de l'espace des phases, Actes de la conférence "Epistémologie des systèmes dynamiques", Paris, décembre 1999, in Chaos et systèmes dynamiques : Eléments pour une épistémologie, S. Franceschelli, M. Paty \& T. Roque ed., Hermann (2007).

[C2] A. Chenciner, Une note de Poincaré, Proceedings Curs Poincaré Barcelona, 2005, English translation A note by Poincaré, Regular and Chaotic Dynamics, V. $10 n^{\circ} 2,2005,119-128$.

[C3] A. Chenciner, Une promenade dans les Méthodes Nouvelles de la Mécanique Céleste, Gazette des Mathématiciens no 134, (octobre 2012) 37-47.

[C4] A. Chenciner, Three-Body Problem, Scholarpedia, http://www . scholarpedia.org/article/Three_body_problem.

[C5] A. Chenciner, Four Lectures on the N-body problem, in Hamiltonian Dynamical Systems and Applications, W. Craig ed., Springer (2008), 21-52.

[C6] A. Chenciner, Compte-Rendu de lecture du premier volume des œuvres complètes de Jean Le Rond D'Alembert (premiers textes de mécanique céleste 1747-1749), Gazette des Mathématiciens nº 99, (janvier 2004) 107-114.

[C7] A. Chenciner, À l'infini en temps fini, Sém. Bourbaki, exposé no 832, juin 1997. http://www . numdam.org/numdam-bin/feuilleter?id=SB_1996-1997__39_.

[C8] A. Chenciner, Solutions du problème des n corps joignant deux configurations : l'idée de Christian Marchal et ce qui s'en suit, Gazette des Mathématiciens n ${ }^{o}$ 99, (janvier 2004) 5-12. 
[C9] A. Chenciner, La dynamique au voisinage d'un point fixe elliptique conservatif: de Poincaré et Birkhoff à Aubry et Mather, Sém. Bourbaki, 622, février 1994 http://www. numdam.org/numdam-bin/feuilleter?id=SB_1983-1984__26_.

[Chi] L. Chierchia, The planetary N-body problem, in Celestial Mechanics, edited by A. Celletti, Vol. 6.119.55 (2012) Encyclopedia of Life Support Systems (EOLSS), Developed under the Auspices of the UNESCO, Eolss Publishers.

[CP] L. Chierchia and G. Pinzari, The planetary N-body problem: symplectic foliation, reductions and invariant tori, Invent math (2011) 186:177.

[Com1] T. Combot, Non-intégrabilité algébrique et méromorphe de problèmes de $n$ corps et de potentiels homogènes de degré -1, Thèse Paris, décembre 2012.

[Com2] T. Combot, A note on algebraic potentials and Morales-Ramis theory, preprint 2012.

[Co] C. Conley, On Some New Long Periodic Solutions of the Plane Restricted Three-body Problem, Comm. Pure and Appl. Math., vol. XVI (1963), 449-467.

See also [C5] (section 1) and Le problème de la lune et la théorie des systèmes dynamiques, a course given by the author in 1985, available at http://www . imcce.fr/Equipes/ASD/person/chenciner/chen_preprint.php

[CZ] C. Conley \& E. Zehnder, The Birkhoff-Lewis fixed point theorem and a conjecture of V. I. Arnold, Invent. Math. 73 (1983), p. 33-49.

[D] G. Darboux, Éloge historique de Henri Poincaré, lu dans la séance publique annuelle du 15 démbre 1913, Académie des Sciences, Institut de France.

[Da] G. Darwin, Periodic orbits, Acta Mathematica 21 (1897) 99-242.

[En] Encyclopédie ou dictionnaire raisonné des sciences, des arts et des métiers, volume 9, article Lune (1765 but this article was written by d'Alembert in 1759).

[Fe1] J. Féjoz, Global Secular Dynamics in the Planar Three-body Problem, Celestial Mechanics and Dynamical Astronomy 84, 159-195 (2002).

[Fe2] J. Féjoz, The N-body problem, in Celestial Mechanics, edited by A. Celletti, Vol. 6.119.55 (2012), Encyclopedia of Life Support Systems (EOLSS), Developed under the Auspices of the UNESCO, Eolss Publishers.

[Fe3] J. Féjoz, On Arnold's theorem in celestial mechanics - a summary with an appendix on the Poincaré coordinates, preprint (2012)

[Fe4] J. Féjoz, Démonstration du "théorème d'Arnold" sur la stabilité du système planétaire (d'après Michael Herman), Michael Herman Memorial Issue, Ergodic Theory Dyn. Sys. 24:5 (2004) 1521-1582.

[FGKR] J. Féjoz, M. Guardia, V. Kaloshin, P. Roldan, Diffusion along mean motion resonance in the restricted planar three-body problam, preprint 2001, http: //www. ceremade.dauphine.fr/ fejoz/articles.php.

[Fey] R. P. Feynman, Lectures in Physics, Vol. I, Addison Wesley 1963. 
[GK] J. Galante and V. Kaloshin, Destruction of invariant curves in the restricted circular planar Three-Body Problem by using comparison of action, Duke Math. J. Volume 159, Number 2 (2011), 275-327.

[GSV] V. Gelfreich, C. Simó and A. Vieiro, Dynamics of 4D symplectic maps near a double resonance, preprint http://www.maia.ub.es/dsg/2012/ (number 5).

[G] E. Ghys, Variations autour du théorème de récurrence de Poincaré, Journal des élèves de l'ENS Lyon no 1 (1994), available at http://www.umpa.ens-lyon. fr/JME/Vol1Num1/artEGhys/artEGhys.html.

[Go] E. Goursat, Sur les transformations isogonales en Mécanique, Comptes Rendus des Séances de l'Académie des Sciences, Paris, 108, 446-448 (1889).

[Gu] M. Gutzwiller, Moon-Earth-Sun: The oldest Three-Body Problem, Reviews of Modern Physics, vol. 70, no 2, (April 1998).

[Ha] J. Hadamard, Les surfaces à courbures opposées et leurs lignes géodésiques, J. Math. Pures Appl. 4 (1898), 27-73.

[HK] Hasselblatt and A. Katok, Modern theory of Dynamical Systems, Cambridge University Press, 1996.

[He] M. Hénon, Generating families in the Restricted Three-body Problem, Lecture Notes in Physics: Monographs 52, Springer 1997.

[Her] M. Herman, Some open problems in dynamical systems, ICM Berlin 1998, II, 797-808.

[JT] E. Julliard-Tosel, Bruns theorem: the proof and some generalizations, Celestial Mechanics and Dynamical Astronomy 76: 241-281 (2000).

[K] A. N. Kolmogorov, On the Conservation of Conditionnally Periodic Motions under Small Perturbations of the Hamiltonian, Dokl. akad. nauk SSSR, 1954, vol. 98, pp. 527-530.

[Koz] V. V. Kozlov, Integrability and non-integrability in Hamiltonian mechanics, Uspechi Mat. Nauk 38:1 (1983), 3-67, english translation in Russian Math. Surveys 38:1 (1983), 1-76.

[Las1] J. Laskar, Le système solaire est-il stable ?, Séminaire Poincaré XIV (2010) 221-246. http://www . bourbaphy .fr/laskar .pdf. Is the Solar System Stable?, in Chaos, Poincaré Seminar 2010, B. Duplantier and V. Rivasseau eds., Progress in Mathematical Physics, Springer Basel AG 2013 (to appear).

[Las2] J. Laskar, Poincaré et le Chaos dans le Système Solaire, a conference given in Nancy, September 20 (2012). http://www.poincare.fr/actualite/ evenements?task=view_event\&event_id=8.

[LasRob] J. Laskar \& P. Robutel, Stability of the planetary Three-Body Problem, I. Expansion of the planetary Hamiltonian, Cel. Mech. 62, 193-217 (1995).

[Laz] V. F. Lazutkin, The existence of caustics for a billiard problem in a convex domain, Math. USSR Izv. 7, 1973, 185-214. 
[Le] P. Le Calvez, Propriétés dynamiques des difféomorphismes de l'anneau et du tore, Astérisque 204 (1991); English transl. Dynamical properties of diffeomorphisms of the annulus and of the torus SMF/AMS Texts \& Monographs, 4.

[LC] T. Levi-Civita, Sur la régularisation du problème des trois corps, Acta Math., vol. 42, 99-144 (1920).

[LS] J. Llibre and C. Simó, Oscilatory Solutions in the Planar Restricted ThreeBody Problem, Math. Ann. 248, 153-184 (1980).

[Lya] A. Lyapunov, Obchtchaia zadatcha ob ouctoïtchivosti dvijenia, Kharkov 1892, French translation under the name of A. Liapounoff Problème général de la stabilité du mouvement, Annales de la faculté des sciences de Toulouse 2ème série, tome 9 (1907), p. 203-474, available at http://afst.cedram.org/item?id= AFST_1907_2_9__203_0, English translation General Problem of the Stability of Motion, CRC Press (1992).

[Mal] B. Malgrange, On nonlinear differential Galois theory, dedicated to the memory of Jacques-Louis Lions, Chinese Ann. Math. Ser. B 23 (2002), no. 2, 219226.

[MRL] F. Malige, P. Robutel, J. Laskar, Partial reduction in the $N$-body planetary problem using the angular momentum, Cel. Mech. Dyn. Astron. 84: 283316, 2002.

[Ma1] C. Marchal, The Three-Body Problem, Elsevier (1990).

[Ma2] C. Marchal, How the method of minimization of action avoids singularities, Celestial Mech. Dynam. Astronom. 83 (2002), no. 1-4.

[McG] R. McGehee, Singularities in Classical Celestial Mechanics, Proceedings of the International Congress of Mathematicians, Helsinki 1978, 827-834.

[Moe] R. Moeckel, Some Qualitative Features of the Three-Body Problem, Contemporary Mathematics 81, 1-22 (1988).

[Mont1] R. Montgomery A New Solution to the Three-Body Problem, Notices of the American Mathematical Society, 471-481, May. (2001)

[Mont2] R. Montgomery N-body choreographies, Scholarpedia, http://www . scholarpedia.org/article/N-body_choreographies

[MS] J. J. Morales-Ruiz and S. Simon, On the meromorphic non-integrability of some N-body problems, DCDS-A, 24(4):12251273, 2009.

[MR] J.J. Morales-Ruiz and J .P. Ramis, Galoisian obstructions to integrability of Hamiltonian systems. I, II, Methods Appl. Anal. 8 no 1, 3395, 97111 (2001).

[MRS] J. J. Morales-Ruiz, J. P. Ramis, and C. Simó, Integrability of Hamiltonian systems and differential Galois groups of higher variational equations, Annales scientifiques de l'École normale supérieure, 40(6):845884, 2007.

[M1] J. Moser, On invariant curves of area preserving mappings of an annulus, Nachr. Akad. Wiss. Gött., Math. Phys. Kl. (1962), 1-20.

[M2] J. Moser, Convergent Series Expansions for Quasi-Periodic Motions, Math. Annalen 169, 1967, 136-176. 
[M3] J. Moser, Stability theory in celestial mechanics, in The stability of the solar system and of small stellar systems, p. 1-9, Kozai ed., I.A.U. (1974).

[M4] J. Moser, Is the Solar System Stable?, The Mathematical Intelligencer, 1 (1978), 65-71.

[M5] J. Moser, Stable and random Motion in dynamical systems, Princeton University Press (1973).

[Na] P. Nabonnand, Les premières contributions d'Henri Poincaré en mécanique céleste vues à partir de sa correspondance avec Anders Lindstedt (1883-1884), a lecture at the Bureau des Longitudes, Paris, October 3, 2012, HAL-00743645.

[Ne] N. N. Nekhoroshev, An exponential estimate of the time of stability of nearly integrable Hamiltonian systems, II. Trudy Sem. Petrovsk., 5:550, 1979.

[N] Sir I. Newton, Opticks or a Treatise on the Reflections, Refractions, Inflections and Colours of Light.

[Nie] L Niedermann, Stability over exponentially long times in the planetary problem, Nonlinearity, 9(6):17031751, 1996.

[Pa] P. Painlevé, Leçons sur la théorie des équations différentielles professées à Stockholm (septembre, octobre, novembre 1895), Hermann, Paris, 1897.

[P1] H. Poincaré, Sur le problème des trois corps et les équations de la dynamique, withdrawn version 1889 and Acta Mathematica 13, 1890, p. 1-270.

[P2] H. Poincaré, Les méthodes nouvelles de la mécanique céleste, (1892, 1893, 1899), Gauthier-Villars, Paris; Russian translation with commentaries by Arnold, Alexeiev, Merman, Pogrebiskii, Moscow 1971; English translation with commentaries by D. Goroff, New Methods of Celestial Mechanics, American Institute of Physics (1992).

[P3] H. Poincaré, Mémoire sur les courbes définies par une équation différentielle, 4 parts 1881, 1882, 1885, 1886, in Euvres, tome I (the title of the two last parts is Sur les courbes définies par les équations différentielles).

[P4] H. Poincaré, Sur certaines solutions particulières du problème des trois corps, Bulletin astronomique, t. 1, p. 65-74 (février 1884).

[P5] H. Poincaré, Sur les solutions périodiques et le principe de moindre action, C.R.A.S. 1896, t. 123, 915-918; in Euvres, tome VII.

[P6] H. Poincaré, Le problème des trois corps, Revue générale des sciences pures et appliquées 2, 1-5 (1891).

[P7] H. Poincaré, Sur les petits diviseurs dans la théorie de la Lune, Bulletin astronomique. V. 25. (1908) p. 321360 [CEuvres tome 8. p. 332366].

[P8] H. Poincaré, Sur la stabilité du système solaire, Annuaire pour l'an 1898 du Bureau des Longitudes, Gauthier-Villars et fils 1897, reproduit dans la Revue scientifique (revue rose), $4^{\text {ème }}$ série, tome IX, 20, 14 mai 1898.

[P9] H. Poincaré, Sur la méthode de Bruns, C.R.A.S. 1896, t. 123, 1224-1228. 
[P10] H. Poincaré, Les solutions périodiques et le principe de moindre action, C.R.A.S. 1897, t. 124, 713-716.

[P11] H. Poincaré, Sur la façon de grouper les termes des séries trigonométriques qu’on rencontre en Mécanique céleste, Bulletin astronomique , t. 15, p. 289-310, aout 1898.

[P12] H. Poincaré, Leçons de mécanique céleste, Gauthier-Villars (1905 1907, 1910).

[P13] H. Poincaré, La valeur de la science, Bibliothèque de philosophie scientifique, Flammarion 1905; English translation The value of science, Dover 1958.

[P14] H. Poincaré, Sur les lignes géodésiques des surfaces convexes, Transactions of the American Mathematical Society, t. 6, p. 237-274 (july 1905).

[P15] H. Poincaré, Sur un théorème de géométrie, Rendiconti del Circolo matematico di Palermo, t. 33, p. 375-407 (1912).

[PC] La Correspondance de Henri Poincaré, Vol. 3 : La Correspondance entre Henri Poincaré et les astronomes et géodésiens, Ralf Krömer, Philippe Nabonnand, Martina Schiavon \& Scott Walter ed., Basel: Birkhäuser, to appear in 2013.

[Pos] J. Pöschel, Integrability of Hamiltonians on Cantor sets. Communications in Pure and Applied Math. XXXV, p. 653-695 (1982).

[Ra1] J. P. Ramis, Poincaré et les développements asymptotiques, (1 ${ }^{e ̀ r e}$ partie), Gazette des mathématiciens 133, juillet 2012, p. 33-72.

[Ra2] J. P. Ramis, Les développements asymptotiques après Poincaré: continuité et... divergences, Gazette des mathématiciens 134, octobre 2012, p. 17-36.

[Ro] A. Robadey, Différentes modalités de travail sur le général dans les recherches de Poincaré sur les sytèmes dynamiques, Thèse Paris 2006.

[Rob] P. Robutel, Stability of the planetary Three-Body Problem II, KAM theory and existence of quasi-periodic motions, Celestial Mech. Dynam. Astron. 62 (1995) 219-261.

[Sau] D. Sauzin, Résurgence paramétrique et exponentielle petitesse de l'écart des séparatrices du pendule rapidement forcé, Thèse, Paris 1994.

[SM] C.L. Siegel and J.K. Moser, Lectures on Celestial Mechanics, Grundlehren der mathematischen Wissenschaften. Springer Verlag (1971).

[Si] C. Simó, Some properties of the global behaviour of conservative low dimensional systems, in Foundations of Computational Mathematics: Hong Kong 2008, F. Cucker et al. editors, pp. 163-189, London Math. Soc. Lecture Notes Series 363, Cambridge Univ. Press, 2009.

[SST] C. Simó, P. Sousa-Silva and M. Terra, Domains of Practical Stability near L4,5 in the 3D Restricted Three-Body Problem, Slides of the lecture given at Dynamical Systems: 100 years after Poincaré, Gijón (Spain) 3-7 September 2012, http://www.maia.ub.es/dsg/2012/ (number 8). 
[ST] C. Simó and T.J. Stuchi, Central stable/unstable manifolds and the destruction of KAM tori in the planar Hill problem, Physica D: Nonlinear Phenomena, Volume 140, Issues 12, 1 June 2000, pages 1-32.

[Sin] Ya. G. Sinai, Introduction to ergodic theory, Erevan state University (in Russian) 1973, English transl.: Mathematical Notes, Princeton Univ. Press, 1977.

[Sm1] S. Smale, Diffeomorphisms with many periodic points, Differential and Combinatorial Topology, Princeton Univ. Press, 1965, pp. 63-80.

[Sm2] S. Smale, Differentiable Dynamical Systems, Bull. Amer. Math. Soc. 73 (1967), 747-817.

[Sz] V. Szebehely, The Theory of Orbits, Academic Press (1967).

[Th] R. Thom, Prédire n'est pas expliquer, Eshel, Paris (1991).

[Ti] Tisserand, Traité de Mécanique céleste, Gauthier-Villars, vol 1: 1889, vol. 2: 1891, vol. 3: 1894, vol. 4: 1896.

[V] C. Villani, (Ir)réversibilité et entropie, Séminaire Poincaré XV, Le temps (2010). http://www.bourbaphy.fr/villani.pdf. (Ir)reversibility and Entropy, in Time, Poincaré Seminar 2010, B. Duplantier ed., Progress in Mathematical Physics, vol. 63, pp. 19-79, Springer Basel AG (2013). (Ir)réversilbilité et entropie, in Time, Poincaré Seminar 2010, B. Duplantier ed., Progress in Mathematical Physics, vol. 63, pp.81-144, Springer Basel AG (2013).

[Wi] A. Wintner, The Analytical Foundations of Celestial Mechanics, Princeton University Press (1949).

[Y] J. C. Yoccoz, Une erreur féconde du mathématicien Henri Poincaré, Gazette des mathématiciens, $\mathrm{n}^{\circ} 107,2006$, p. 19-26.

[Ze] E. Zehnder, Homoclinic Points Near Elliptic Fixed Points, Communications on pure and applied mathematics, Vol. XXVI, 131-182 (1973).

[Z] S. L. Zyglin, Branching of solutions and nonexistence of first integrals in Hamiltonian mechanics, i, Functional Analysis and Its Applications, 16(3) 181-189 (1982). 